\title{
Swampland geometry and the gauge couplings
}

\author{
Sergio Cecotti \\ SISSA, via Bonomea 265, Trieste I-34100, Italy \\ E-mail: cecotti@sissa.it
}

ABSTRACT: The purpose of this paper is two-fold. First we review in detail the geometric aspects of the swampland program for supersymmetric $4 \mathrm{~d}$ effective theories using a new and unifying language we dub "domestic geometry", the generalization of special Kähler geometry which does not require the underlying manifold to be Kähler or have a complex structure. All 4d SUGRAs are described by domestic geometry. As special Kähler geometries, domestic geometries carry formal brane amplitudes: when the domestic geometry describes the supersymmetric low-energy limit of a consistent quantum theory of gravity, its formal brane amplitudes have the right properties to be actual branes. The main datum of the domestic geometry of a 4d SUGRA is its gauge coupling, seen as a map from a manifold which satisfies the geometric Ooguri-Vafa conjectures to the Siegel variety; to understand the properties of the quantum-consistent gauge couplings we discuss several novel aspects of such "Ooguri-Vafa" manifolds, including their Liouville properties.

Our second goal is to present some novel speculation on the extension of the swampland program to non-supersymmetric effective theories of gravity. The idea is that the domestic geometric description of the quantum-consistent effective theories extends, possibly with some qualifications, also to the non-supersymmetric case.

KEYwords: Differential and Algebraic Geometry, Supergravity Models, Models of Quantum Gravity

ARXiv EPrint: 2102.03205 


\section{Contents}

1 Introduction 1

2 Generalities on effective theories 4

2.1 Our set-up 4

2.2 The $U$-duality group 5

2.3 Singularities of moduli spaces $\quad 6$

$\begin{array}{lll}\text { 2.3.1 Singularities from the action of } \mathcal{G} & 6\end{array}$

2.3.2 Example: Type IIB on a 3-CY 8

$\begin{array}{ll}2.3 .3 & \text { Smoothing surgery }\end{array}$

$\begin{array}{ll}2.4 \text { Behavior at infinity } & 11\end{array}$

$\begin{array}{lll}\text { 2.4.1 Sign of curvature at infinity } & 11\end{array}$

$\begin{array}{lll}2.4 .2 & \text { Cusps } & 12\end{array}$

3 OV manifolds $\quad 14$

$\begin{array}{lll}3.1 & \text { First consequences of the definition } & 15\end{array}$

$\begin{array}{lll}3.1 .1 & \text { Holonomy groups and the graded algebra } \mathcal{P}^{\bullet} & 15\end{array}$

$\begin{array}{lll}3.1 .2 & \text { Liouville property } & 17\end{array}$

4 The gauge couplings $\tau(\phi)_{a b}$ as a map $\quad 17$

$\begin{array}{lll}4.1 \text { Generalities } & 18\end{array}$

$\begin{array}{lll}4.2 & \text { Three different viewpoints on } \tau(\phi)_{a b} & 19\end{array}$

4.3 DG invariants of the gauge couplings $\tau(\phi)_{a b} \quad 20$

$\begin{array}{ll}4.4 & \text { Tension field and harmonic maps } \\ \end{array}$

4.4.1 Energy and tension of maps into symmetric spaces 22

$\begin{array}{lll}\text { 4.4.2 Cartan gauge couplings } & 23\end{array}$

5 Domestic geometry $\quad 24$

5.1 (Arithmetic) Tamed maps 24

$\begin{array}{lll}\text { 5.1.1 Special cases } & 25\end{array}$

$\begin{array}{lll}5.2 & \text { Generalized } t t^{*} \text { geometry } & 26\end{array}$

$\begin{array}{lll}\text { 5.2.1 HIVb brane amplitudes } & 29\end{array}$

5.2.2 Graded $t t^{*}$ geometries \& VHS 31

5.2.3 Explicit graded HIVb brane amplitudes $\quad 34$

5.2.4 Physical quantities from brane amplitudes 34

5.2.5 $t t^{*}$ entropy functions \& Mumford-Tate groups 34

$\begin{array}{lll}5.3 & \text { Domestic geometry } & 36\end{array}$

5.3.1 Example: 2d SCFT 36

$\begin{array}{lll}\text { 5.3.2 Domestic brane amplitudes } & 37\end{array}$

$\begin{array}{lll}5.4 & \text { Entropy functions in domestic geometry } & 37\end{array}$ 
6 Domestic geometry and supergravity

$\begin{array}{lll}6.1 & \text { Supergravity in } 4 \mathrm{~d} & 38\end{array}$

6.2 Swampland vs. brane amplitudes $\quad 41$

6.3 Supergravity as moduli-space gravitational instantons 41

7 Domestic geometry and the swampland $\quad 42$

7.1 Domestic geometry vs. OV manifolds 43

7.2 Tension flow and naturalness of the gauge couplings 44

$\begin{array}{lll}7.2 .1 & \text { Tension flow } & 44\end{array}$

7.2.2 Relation to Ricci flow $\quad 45$

7.2.3 Naturalness of the effective Lagrangian $\mathscr{L}_{\text {eff }} \quad 46$

$\begin{array}{lll}7.2 .4 & \text { Naturalness: adding gravity } & 47\end{array}$

$\begin{array}{lll}7.2 .5 & \text { A word of caution } & 49\end{array}$

7.3 Existence, uniqueness, and structure of tamed maps 50

$\begin{array}{lll}\text { 7.3.1 Compact source space } & 50\end{array}$

$\begin{array}{lll}\text { 7.3.2 The case of OV manifolds } & 58\end{array}$

$\begin{array}{lll}\text { 7.3.3 Structure of the gauge coupling } \mu & 60\end{array}$

7.3.4 Swampland conditions for $\mathcal{N}=2$ SUGRA $\quad 61$

$\begin{array}{lll}7.4 & \text { First applications } & 62\end{array}$

$\begin{array}{ll}\text { A SUGRA spaces: tamed maps vs. special holonomy } & 63\end{array}$

$\begin{array}{ll}\text { B No boundary term in the Bochner argument } & 65\end{array}$

\section{Introduction}

The swampland program [1,2] (for reviews see [3, 4]) aims to characterize the sparse subset of effective field theories which arise as low-energy limits of consistent theories of quantum gravity inside the much larger set of formal theories which "look" consistent from a low-energy perspective, but cannot be completed to a fully consistent theory of quantum gravity. A model which cannot be consistently completed is said to belong to the swampland.

The program has taken the form of a rapidly growing list of conjectural necessary conditions (the "swampland conjectures" [1-4]) that all effective theories of quantum gravity should obey. These conditions are motivated by general physical considerations (in particular the thermodynamics of black holes [5]) as well as by lessons drawn from the large supply of consistent effective theories of gravity which describe the light degrees of freedom in a controlled vacuum of superstring theory (the string lamppost principle (SLP) [6]). We have full analytic control on the quantum stability of a candidate string vacuum only when it preserves some supersymmetry, and we can write a precise effective Lagrangian only 
when its couplings are protected by a SUSY non-renormalization theorem: so all reliable examples at our disposal have extended supersymmetry. ${ }^{1}$

The best understood examples are the effective theories of Type IIB compactified to $4 \mathrm{~d}$ on some Calabi-Yau (CY) 3-fold: these examples played a major role in the development of the swampland program [2]. The resulting low-energy $4 \mathrm{~d} \mathcal{N}=2$ supergravities are described (in the vector sector) by special Kähler geometry [7, 8]. Special Kähler geometry is a very powerful tool to study the quantum consistency of an $\mathcal{N}=2$ supergravity; a significant part of the swampland program $[9-15,18,19]$ is dedicated to the detailed analysis of the "motivic" special Kähler manifolds which describe the moduli geometry of actual CY manifolds (or motives). In our opinion, the swampland program has reached a rather satisfactory shape in this specific $\mathcal{N}=2$ set-up and "motivic" special Kähler geometry must be seen as a successful model of swampland theory. It would be highly desirable to extend this model theory to broader contexts.

Supersymmetric effective theories are better behaved in many ways, and SUSY seems to play a crucial role in the consistency of many explicit examples. In some contexts [20] it is hard to grasp how a model can possibly be consistent without being supersymmetric.

On the other hand, the real world does not look supersymmetric at low energy, and hence consistent non-supersymmetric effective theories of gravity ought to exist. An important goal of the swampland program is to say something less vague about the non-SUSY effective theories of consistent quantum gravity. They are expected to be quite rare and remarkable animals, in a sense even more "magical" than their SUSY counterparts. Morally speaking, these theories should enjoy "all the good properties" of SUSY while avoiding its phenomenological drawbacks as the existence of (unobserved) super-partners. We should not expect such "magical" theories to share the properties which generically hold for a non-SUSY field theory, because they are extremely non-generic. From this point of view, the highly non-generic value of the real-world effective cosmological constant $\Lambda$ is hardly a surprise.

The author's own prejudice is that there should be a "more general" swampland principle, which under appropriate circumstances reduces to (or implies) SUSY, but which continues to make sense in some very restricted non-supersymmetric context where most of the "good" facts about SUSY still hold. The reader will find many echoes of the prejudice in this paper.

The purpose of this paper is two-fold. First we present a systematic review of the geometric aspects of the swampland program in the SUSY context from a novel unifying perspective. The two key concepts of this approach are:

(i) Ooguri-Vafa (OV) manifolds, i.e. the Riemannian spaces with the correct properties (according to $[1,2]$ ) to be the scalars' spaces of a consistent effective theory;

(ii) domestic geometry, with its brane amplitudes and generalized entropy functions.

Domestic geometry is the direct generalization of special Kähler geometry which does not require the underlying manifold to carry a complex structure. All 4d supergravities are

\footnotetext{
${ }^{1}$ This limitation in the available explicit examples may a priori lead to some bias in our understanding of quantum gravity.
} 
described by a domestic geometry in the same exact way that the vector-multiplet sector of $4 \mathrm{~d} \mathcal{N}=2$ SUGRA is described by the usual special Kähler geometry. In particular, the vectors' couplings have an universal expression in terms of domestic brane amplitudes. Domestic geometry looks particularly deep and natural when the underlying manifold is OV. Combining the two ingredients (i), (ii) one gets arithmetic domestic geometry which is the obvious domestic generalization of the class of "motivic" special Kähler geometries which describe the vector-sector of quantum-consistent $4 \mathrm{~d} \mathcal{N}=2$ supergravities. This allows to phrase the swampland conditions for the general SUSY model in the same suggestive language used in the $\mathcal{N}=2$ case [15]:

A $4 \mathrm{~d}$ supergravity is described by a domestic geometry from which we compute formal brane amplitudes. If the supergravity is not in the swampland, the formal amplitudes describe actual branes.

OV manifolds have several nice properties. In particular, they are Liouvillic for the sub-harmonic functions. This entails that the rigidity properties of "motivic" special Kähler geometry (the "power of holomorphy" [21]) hold for all OV manifolds even if they do not carry any complex structure.

Our second main purpose is to present some novel speculations about the swampland conditions in the non-SUSY case. The space of light scalars $\mathscr{M}$ is (conjecturally) an Ooguri-Vafa manifold in all quantum-consistent effective theories of gravity, supersymmetric or not. Arithmetic domestic geometry is naturally defined on all OV manifolds, and its statements make perfect sense for non-SUSY effective Lagrangians. One is then led to speculate that domestic geometry — which applies to all SUSY cases - may also be relevant to describe vectors' couplings in quantum-consistent non-SUSY $4 \mathrm{~d}$ effective theories. The speculation may be stated at different levels of precision. Domestic geometry may be: (a) just qualitatively valid, or (b) semi-quantitatively correct, or even (c) exact. Besides its aesthetic geometric appeal, and the evidence from SUSY examples, the speculation rests on some heuristic physical arguments, based on the idea of "naturalness" in the IR description, which suggest that at least version (b) should hold.

Warnings to the reader. The materials presented in this paper are of different nature and logical status. There are: (a) mathematically rigorous geometric constructions/results; (b) physical statements which aim only to a physicists' level of rigor; (c) widely accepted swampland conjectures (which are taken as "facts" in most of the recent literature); (d) newly proposed swampland conjectures which are still open to discussion by the community; (e) speculative statements which are meant as an invitation to further work in the hope of re-formulating them as precise swampland conjectures in the near future. To make the distinction clear to the reader, statements related to (a) are labelled theorem, lemma, and so. Most statements in the logical classes (b) and (c) are dubbed facts. New conjectures and speculations are called by their proper name.

In the paper we often use the phrase: "[this statement is] true in all known examples of reliable quantum-consistent effective theories of gravity" or similar ones. Since there is an ongoing debate in the community about which examples should be considered "reliable", we need to specify the class of examples we have in mind. Our notion of reliability is the 
most conservative one, that is, we restrict to examples which are under full analytic control and are pacifically considered "reliable" by all experts in the field.

Organization of the paper. The rest of this paper is organized as follows. In section 2 we discuss some general properties of $4 \mathrm{~d}$ effective field theories and some technical aspects of the singularities and asymptotics of the moduli spaces. In section 3 we define the OV manifolds and discuss their basic properties. In section 4 we describe the gauge couplings seen as a map from the moduli space to the Siegel variety. Section 5 contains the basics of domestic geometry; a large part of the section is a detailed review of (generalized) $t t^{*}$ geometry which is the model which inspires all constructions in domestic geometry. Section 6 describes how domestic geometry applies to all 4d SUGRAs and may be used to reformulate the swampland conditions in the SUSY context in a more convenient way. Section 7 describes in more detail domestic geometry, presenting the math arguments for the existence and uniqueness of the underlying tamed maps, and rephrasing them as heuristic physical arguments in favour of our speculation that arithmetic domestic geometry is relevant for the non-SUSY swampland program. Some additional technicality is confined in the appendices.

Remark 1. The recent papers $[16,17]$ describe a different uniform geometric approach to supergravity, dubbed bosonic supergravity. It would be interesting to understand geometric aspects of the swampland conditions from that perspective.

\section{Generalities on effective theories}

\section{$2.1 \quad$ Our set-up}

To make the story a bit shorter, in this paper we consider only four-dimensional effective theories. Although methods and ideas apply to much wider contexts, we assume a vanishing cosmological constant, $\Lambda=0$, and focus on effective Lagrangians valid at parametrically small energies. In particular, we make the following two assumptions:

A1 all visible IR gauge degrees of freedom (d.o.f.) are in their Coulomb phase. Locally at generic points in field space we may choose an electro-magnetic duality frame, with respect to which these d.o.f. are described by Abelian gauge vectors $A^{a}(a=1, \ldots, h)$ with field strengths $F^{a}=d A^{a}$;

A2 the (exactly) massless fields carry no electric or magnetic charge under the $A^{a}$ 's.

The light degrees of freedom then consist of a space-time metric $g_{\mu \nu}, h$ vector fields $A^{a}$, and $m$ massless real scalars $\phi^{i}$, together with spin- $1 / 2$ fermions and possibly spin-3/2 gravitini (only in the SUSY case). In the Einstein frame (and the chosen electro-magnetic frame) the effective Lagrangian takes (locally in field space) the form

$$
\mathscr{L}_{\text {eff }}=-\sqrt{-g}\left(\frac{1}{2} R+\frac{1}{2} G(\phi)_{i j} \partial^{\mu} \phi^{i} \partial_{\mu} \phi^{j}-\frac{i}{16 \pi} \tau(\phi)_{a b} F_{+}^{a} F_{+}^{b}+\frac{i}{16 \pi} \bar{\tau}(\phi)_{a b} F_{-}^{a} F_{-}^{b}+\cdots\right)
$$

where $^{2} F_{ \pm}^{a}=\frac{1}{2}(1-\mp i *) F^{a}$ is the (anti-)self-dual part of the field-strength $F^{a}$.

\footnotetext{
${ }^{2}$ We use the shorthand $F_{ \pm}^{a} F_{ \pm}^{b} \equiv\left(F_{ \pm}^{a}\right)_{\mu \nu}\left(F_{ \pm}^{b}\right)^{\mu \nu}$.
} 
The swampland program asks for a characterization of the field-dependent couplings in the Lagrangians $\mathscr{L}_{\text {eff }}$ which describe low-energy limits of consistent quantum gravities. In practice, one looks for necessary conditions they should satisfy, which are usually phrased as sufficient conditions for the model (2.1) to sink in the swampland.

In this paper we limit ourselves to the geometric part of the swampland program, i.e. to the two-derivative couplings $G(\phi)_{i j}$ and $\tau(\phi)_{a b}$.

The characterization of the consistent scalars' kinetic couplings $G(\phi)_{i j}$ takes advantage from their geometrical interpretation [2]. The scalar fields $\phi^{i}$ are seen as local coordinates on a connected "manifold" $\widetilde{\mathscr{M}}$, of dimension $m$, endowed with the Riemannian metric $G_{i j} \equiv G(\phi)_{i j}$. The Riemannian metric is smooth at generic points of $\widetilde{\mathscr{M}}$.

Remark 2. In a generic non-SUSY effective field theory we do not expect a non-trivial space $\widetilde{\mathscr{M}}$ of exactly massless scalars since the flat directions of the potential are usually lifted by quantum effects. This needs not to apply in the present context, since quantumconsistent theories of gravity are highly non-generic. On the other side, the conclusions of this paper remain valid even if the scalars parametrizing $\widetilde{\mathscr{M}}$ are not exactly massless but only hierarchically lighter than the scale above which the low-energy description breaks down.

\subsection{The $U$-duality group}

A basic datum of the effective field theory is its gauge group. The discrete part of the gauge group is called the $U$-duality group $\mathcal{G} . \mathcal{G}$ is a redundancy of the description, so it is tautologically an exact symmetry of the full quantum theory not just of its IR sector. More precisely, by $\mathcal{G}$ we mean the quotient of the full discrete gauge group of the underlying UV complete theory which acts faithfully on the light bosonic fields. In particular, $\mathcal{G}$ acts on fluxes of forms of various degrees $k$

$$
\frac{1}{2 \pi} \oint_{\Sigma_{k}} F^{(k)}
$$

which are integral by generalized Dirac quantization: the fluxes (2.2) take value in a lattice $\Lambda$ endowed with a non-degenerated bilinear form $\Lambda \otimes \Lambda \rightarrow \mathbb{Z}$ (the generalized Dirac pairing). The completeness conjecture [5] states that in a consistent theory all fluxes allowed by Dirac quantization are realized by some physical state. In $4 \mathrm{~d}$ the electro-magnetic charges take value in a lattice $V_{\mathbb{Z}} \cong \mathbb{Z}^{2 h}$ with a skew-symmetric principal ${ }^{3}$ Dirac pairing. The action of $\mathcal{G}$ on the scalar fields and the electro-magnetic fluxes defines a group embedding ${ }^{4}$

$$
\mathcal{G} \hookrightarrow \operatorname{Iso}(\widetilde{\mathscr{M}}) \times \operatorname{Sp}(2 h, \mathbb{Z}) .
$$

The kernel of the map $\mathcal{G} \rightarrow \operatorname{Iso}(\widetilde{\mathscr{M}})$ is finite $;{ }^{5}$ since we always work modulo finite groups, ${ }^{6}$ we shall not distinguish $\mathcal{G}$ from its image in $\operatorname{Iso}(\widetilde{\mathscr{M}})$. The map $\rho: \mathcal{G} \rightarrow \operatorname{Sp}(2 h, \mathbb{Z})$ is called

\footnotetext{
${ }^{3}$ Principal means that the Dirac pairing $V_{\mathbb{Z}} \otimes V_{\mathbb{Z}} \rightarrow \mathbb{Z}$ yields an identification $V_{\mathbb{Z}} \equiv V_{\mathbb{Z}}^{\vee}$.

${ }^{4} \operatorname{Iso}(\widetilde{\mathscr{M}})$ is the isometry group of $\widetilde{\mathscr{M}}$ with metric $G(\phi)_{i j}$.

${ }^{5}$ Indeed the kernel is both compact and discrete.

${ }^{6}$ The symbol $\sim$ stands for equivalence up to commensurability [22] i.e. equality up to finite groups.
} 
the monodromy representation, and its image $\Gamma \subset \mathrm{Sp}(2 h, \mathbb{Z})$ the monodromy group. Hence the $U$-duality group $\mathcal{G}$ is a group extension

$$
1 \rightarrow \mathcal{G}^{0} \rightarrow \mathcal{G} \rightarrow \Gamma \rightarrow 1
$$

that is, $\mathcal{G} \cong \mathcal{G}^{0} \rtimes \Gamma$. A version of the $\pi_{1}$-conjecture [2], which holds true in all known examples, states that the $U$-duality group is isomorphic to a subgroup $\mathcal{G} \subset \operatorname{GL}(k, \mathbb{Z})$ (for some $k$ ) which is generated by $k \times k$ unipotent matrices (modulo finite groups). Thus $\mathcal{G}$ is a subgroup of the $\mathbb{Q}$-algebraic group $\mathrm{GL}(k, \mathbb{Q})$, and in all examples ${ }^{7}$ its $\mathbb{Q}$-Zariski closure $\overline{\mathcal{G}}^{\mathbb{Q}}$ is semi-simple. Hence, modulo finite groups, $\mathcal{G} \sim \mathcal{G}^{0} \times \Gamma$. The moduli space is

$$
\mathscr{M} \equiv \widetilde{\mathscr{M}} / \mathcal{G}
$$

that is, the space of inequivalent (effective) vacua. The covering space $\widetilde{\mathscr{M}}$ may be assumed to be simply-connected with no loss. Then $\pi_{1}(\mathscr{M}) \sim \mathcal{G}$.

\subsection{Singularities of moduli spaces}

The purpose of this technical sub-section is to argue that we may be cavalier with the singularities of the moduli space $\mathscr{M}$, and see in which sense we may work as if $\mathscr{M}$ was a good (三 complete) Riemannian manifold. The sub-section may be omitted in a first reading.

\subsubsection{Singularities from the action of $\mathcal{G}$}

We write $d(\cdot, \cdot): \widetilde{\mathscr{M}} \times \widetilde{\mathscr{M}} \rightarrow \mathbb{R}_{\geq 0}$ for the distance function defined by the metric $G_{i j}$ and replace $\widetilde{\mathscr{M}}$ by its metric completion. Fix a non-trivial unipotent element $u \in \mathcal{G}$; the $\pi_{1}$-conjecture $[2,3]$ says that the infimum of the function

$$
d_{u}: \widetilde{\mathscr{M}} \rightarrow \mathbb{R}_{\geq 0} \quad d_{u}(x) \stackrel{\text { def }}{=} d(u x, x)
$$

is zero. Assuming the conjecture, we consider a sequence of regular points $\left\{x_{i}\right\} \subset \widetilde{\mathscr{M}}_{\text {reg }}$ such that $d_{u}\left(x_{i}\right) \searrow 0$. We have two possibilities:

(1) $x_{i}$ escapes to infinity, i.e. $d\left(x_{1}, x_{i}\right) \rightarrow \infty$. We say that " $u$ has a fixed point at infinity". The distance conjecture [2] requires an infinite tower of states to become exponentially light as we approach the fixed point at infinity. Their electro-magnetic charges belong to $\operatorname{ker}(\rho(u)-1)$;

(2) $\left\{x_{i}\right\}$ remains inside some finite-radius ball $B\left(x_{1}, r\right)$, and hence contains a subsequence which converges to a finite-distance point $x_{*} \in \widetilde{\mathscr{M}}$ which is fixed by $u$.

In the second case the action of $\mathcal{G}$ yields an obstruction to a smooth extension of the Riemannian metric to $x_{*}$ : indeed its isotropy sub-group $\mathcal{G}_{x_{*}} \subset \mathcal{G} \subset \operatorname{Iso}(\widetilde{\mathscr{M}})$ is discrete and contains the infinite group of isometries $u^{\mathbb{Z}}$, whereas the isotropy sub-group of a regular point in Riemannian geometry is always compact. Hence $x_{*}$ is a finite-distance singularity

\footnotetext{
${ }^{7}$ The statement holds in all SUSY consistent theories by a basic fact in domestic geometry, see section 7.3. The reliable examples are all supersymmetric.
} 
where the curvature blows up. In a quantum-consistent effective theory with $\mathcal{N} \geq 2$ supersymmetry all singularities of the completed covering space $\widetilde{\mathscr{M}}$ are of this kind, i.e. fixed-points of non-trivial unipotent elements $u \in \mathcal{G}$, while the moduli space $\mathscr{M} \equiv \widetilde{\mathscr{M}} / \mathcal{G}$ has, in addition, orbifold quotient singularities. Thus in the extended SUSY case all finiteand infinite-distance singularities of $\mathscr{M}$ are dictated by the action of the $U$-duality group $\mathcal{G}$ - they correspond to vacua where a non-trivial subgroup of $\mathcal{G}$ remains unbroken - and no other "accidental" singularity is present. The logic beyond the $\pi_{1}$ and distance conjectures suggests that this is the case for a general consistent effective gravity theory: all singular points are fixed by some non-trivial subgroup of the discrete gauge group $\mathcal{G}$.

We give a closed look to the two kinds of finite-distance singularities.

Quotient orbifold singularities. In general the $U$-duality group $\mathcal{G}$ does not act freely on the regular locus $\widetilde{\mathscr{M}}_{\text {reg }} \subset \widetilde{\mathscr{M}}$. The isotropy sub-group $\mathcal{G}_{\tilde{x}}$ of a smooth point $\tilde{x} \in \widetilde{\mathscr{M}}_{\text {reg }}$ is finite. The image of such a point in the moduli space $\mathscr{M}$ is then a mild orbifold singularity. Orbifold points correspond physically to vacua where a finite sub-group $\mathcal{G}_{\tilde{x}} \subset \mathcal{G}$ remains unbroken. In some stringy examples such points correspond to vacua with a non-Abelian enhancement of the effective continuous gauge symmetry, i.e. loci where our standing assumptions A1, A2 break down and $\mathscr{L}_{\text {eff }}$ ceases to be a complete description of the IR physics.

When we have an embedding $\iota: \mathcal{G} \rightarrow \mathrm{GL}(k, \mathbb{Z})$ we can "repair" the orbifold singularities in a cheap way. Fix an integer $n \geq 3$; let $r_{n}: \operatorname{GL}(k, \mathbb{Z}) \rightarrow \mathrm{GL}(k, \mathbb{Z} / n \mathbb{Z})$ be the reduction $\bmod n$, and write $\iota_{n} \equiv r_{n} \circ \iota$. Consider the exact sequence of groups

$$
1 \rightarrow \mathcal{G}_{n} \rightarrow \mathcal{G} \stackrel{\iota_{n}}{\longrightarrow} \mathrm{GL}(k, \mathbb{Z} / n \mathbb{Z}) \rightarrow 1 .
$$

It follows from the Minkowski theorem that the matrix group $\mathcal{G}_{n}$ is a normal subgroup of $\mathcal{G}$ of finite index which is torsion-free, in facts neat [22]. This implies that $\mathcal{G}_{n}$ acts freely on $\widetilde{\mathscr{M}}_{\text {reg }}$ so that $\mathscr{M}_{n} \equiv \widetilde{\mathscr{M}} / \mathcal{G}_{n}$ is a finite Galois cover of $\mathscr{M}$, with Galois group $\iota_{n}(\mathcal{G}) \equiv \mathcal{G} / \mathcal{G}_{n}$, free of orbifold singularities. The finite-quotient singularities may be cured by replacing $\mathscr{M}$ with $\mathscr{M}_{n}$ and $\mathcal{G}$ with $\mathcal{G}_{n}$ : this is the standard strategy in the math literature when studying moduli spaces of projective varieties (in particular of Calabi-Yau 3-folds), and we adopt it. From now on by $\mathcal{G}$ we always mean a finite-index, neat, normal subgroup of the actual $U$-duality group. Correspondingly $\mathscr{M} \equiv \widetilde{\mathscr{M}} / \mathcal{G}$ is free of finite-quotient singularities.

Finite-distance curvature singularities. $\widetilde{\mathscr{M}}_{\text {reg }}$ is not geodesically complete in general; that is, $\widetilde{\mathscr{M}}_{\text {reg }}$ may contain half-geodesics $\ell(t)$ originating from a smooth point $\phi_{0} \equiv \ell(0)$ which cannot be continued after some finite value of the proper length $t$. Such a finite-length maximal half-geodesic represents a physical transition - which takes finite time and costs finite energy per unit volume - from our initial configuration $\phi_{0}$ to a physical situation where the IR description provided by $\mathscr{L}_{\text {eff }}$ is no longer valid. This finite-time process should be perfectly regular from the viewpoint of the UV complete theory. What happens is that $\ell(t)$ stops at a vacuum where "new physics" becomes relevant in the infra-red: some additional degrees of freedom of the fundamental UV theory get massless, and our IR description breaks down. From the viewpoint of the UV fundamental theory, these 
singularities typically correspond to points where different branches of the space of vacua meet each other (transition points). Since the process involves a finite energy density, one expects that there exists a refined effective Lagrangian $\mathscr{L}_{\text {new }}$ which includes the relevant "new physics" and is valid up to some higher but still finite energy scale. $\mathscr{L}_{\text {new }}$ allows us to extend the IR description, and hence the physical process described by the half-geodesic $\ell(t)$, beyond the domain $\widetilde{\mathscr{M}}_{\text {reg }}$. This means that family of finite-length half-geodesics in $\left(\widetilde{\mathscr{M}}_{\mathrm{reg}}, G_{i j}\right)$ ending at a given singular point $x_{*}$ is associated to a finite number of new states becoming massless; in addition their spins must be $\leq \frac{3}{2}$. This is to be contrasted with the case of a fixed point at $\infty$, where an infinite tower of states get light [2].

We expect all finite-distance curvature singularities to correspond to fixed points $x_{*}$ under a parabolic subgroup of $\mathcal{G}_{*} \subset \mathcal{G}$ (cfr. the discussion after eq. (2.6)). We have a finite set of vectors $\{q\} \subset V_{\mathbb{Z}}$ which correspond to the electro-magnetic charges of the finitelymany states which becomes massless at $x_{*}$. They should be invariant under $\mathcal{G}_{*}$ (modulo finite groups), i.e. $\rho(u) q=q$ for $u \in \mathcal{G}_{*}$. At such a singularity, the metric is continuous (in appropriate local coordinates) but the curvature invariants blow-up. If our effective theory has $\mathcal{N} \geq 5$ supersymmetry, there is no matter supermultiplets which may become massless, so no finite-distance curvature singularities may be present. The same holds for $\mathcal{N}=3,4$ as a result of a SUSY non-renormalization theorem. ${ }^{8}$

\subsubsection{Example: Type IIB on a 3-CY}

The various kinds of singularities are well illustrated by the vector-scalars' moduli space $\mathscr{M}_{v}$ of Type IIB compactified on a Calabi-Yau 3-fold $X$. The regular locus of its covering space, $\widetilde{\mathscr{M}}_{v \text {, reg }}$, may be identified with the moduli space of marked and polarized CalabiYau's in the smooth deformation class of $X$. $\widetilde{\mathscr{M}}_{v}$, reg is equipped with its Weil-Petersson (WP) Kähler metric $G_{i \bar{j}}$, which is the metric appearing in the scalars' kinetic terms $[7,8]$. Singularities at finite distance in the WP metric correspond to points where there is a conifold transition (more generally, an extremal transition) to a CY with different Hodge numbers [23]. According to a celebrated suggestion by Reid [24], all CY moduli spaces are expected to be connected through such transitions. The full Type IIB string theory remains regular at those transitions, but the low-energy description based on the effective $\mathcal{N}=2$ supergravity breaks down. On $\widetilde{\mathscr{M}}_{v}$, reg there is also another, better behaved, canonical Kähler metric $K_{i \bar{j}}$, the Hodge one (a.k.a. the $K$-metric $[25,26]$ ), which has the expression (with $n=\operatorname{dim}_{\mathbb{C}} \mathscr{M}_{v}$ ) $[25-28]$

$$
\begin{aligned}
& K_{i \bar{j}}=(n+3) G_{i \bar{j}}+R_{i \bar{j}} \geq 2 G_{i \bar{j}}, \\
& R_{i \bar{j}} \geq-(n+1) G_{i \bar{j}},
\end{aligned}
$$

\footnotetext{
${ }^{8}$ The curvature singularity is proportional to the contribution of the gauge coupling beta-function from the states (with e.m. charges $\{q\}$ ) which become massless at $x_{*}$, so it vanishes for $\mathcal{N}=3,4$ where the only matter supermultiplets are vector-multiplets which yield a zero net contribution to the $\beta$-function. Geometrically, this non-renormalization theorem corresponds to the fact that, for $\mathcal{N} \geq 3$ SUGRA, $\widetilde{\mathscr{M}}$ is a symmetric space, whose curvature is parallel, $\nabla_{i} R_{j k l m}=0$, so cannot blow up anywhere.
} 
where $R_{i \bar{j}}$ is the Ricci curvature of the WP metric $G_{i \bar{j}}$. It is important to notice that, while the Ricci curvature ${ }^{9} R_{i \bar{j}}$ of the WP metric satisfies the lower bound (2.9), the Ricci curvature of the $K$-metric $R_{i j}^{K}$ satisfies an upper bound [27]

$$
R_{i \bar{j}}^{K} \leq-\frac{1}{(\sqrt{n}+1)^{2}+1} K_{i \bar{j}}
$$

From eq. (2.8) we see that points at infinite distance in the WP metric are also at infinite distance in the $K$-metric. The opposite statement is false: in terms of the $K$-metric all finite-distance curvature singularities are pushed at infinite distance. Indeed, at conifold points the metric $G_{i \bar{j}}$ remains bounded while the Ricci curvature blows up so that the $K$-metric blows up.

The Torelli space is the completion of $\widetilde{\mathscr{M}}_{v}$ reg with respect to the $K$-metric [29-31]

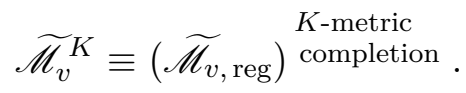

$\widetilde{\mathscr{M}}_{v}{ }^{K}$ is a smooth space diffeomorphic to $\mathbb{R}^{2 m}[29-31]$; since $\mathcal{G}$ is torsion-less, the space

$$
\mathscr{M}_{v}^{K} \equiv \widetilde{\mathscr{M}}_{v}^{K} / \mathcal{G}
$$

is a version of a finite cover of the moduli space which is a smooth Kähler manifold, complete for the $K$-metric, with a contractible universal cover. Its fundamental group $\pi_{1}\left(\mathscr{M}_{v}^{K}\right) \cong \mathcal{G}$ satisfies (a refined version of) the $\pi_{1}$-conjecture [2]. Unfortunately the nice manifold $\mathscr{M}_{v}^{K} \equiv \widetilde{\mathscr{M}}_{v}^{K} / \mathcal{G}$ is not complete for the physical WP metric.

$\mathscr{M}_{v}^{K}$ is the natural space to parametrize the complex structures on a fixed smoothclass of CY 3-folds: $\mathscr{M}_{v}^{K}$ does not talk to moduli spaces of CY's with different topologies which are pushed infinitely away in the $K$-metric. The physical moduli space of Type IIB compactified on $X$ instead consists of several branches of vacua, with extremal transitions between them; since the different branches are not infinitely separated, the physically relevant metric $G_{i \bar{j}}$ on each branch cannot be complete.

There is another description of these moduli spaces more in the spirit of Algebraic Geometry. There is a compact projective variety $\overline{\mathscr{M}}_{v}$ and an effective divisor $D$ such that

$$
\mathscr{M}_{v}^{K}=\overline{\mathscr{M}}_{v} \backslash D .
$$

By Hironaka theorem, we can choose the pair $\left(\overline{\mathscr{M}}_{v}, D\right)$ so that $\overline{\mathscr{M}}_{v}$ is smooth and $D=\sum_{i} D_{i}$ is a simple normal crossing divisor. The points in the support of $D$ are at infinite distance in the $K$-metric. With respect to the WP metric $D$ splits as $D=D_{\infty}+D_{f}$, where $D_{f}$ (resp. $D_{\infty}$ ) is the singular locus at finite (resp. infinite) distance.

\subsubsection{Smoothing surgery}

Returning to the general case, the presence of finite-distance curvature singularities in $\widetilde{\mathscr{M}}$ seems to be unavoidable when $m \geq 2$, unless the low-energy theory is a $\mathcal{N} \geq 3$ supergravity

\footnotetext{
${ }^{9}$ More in detail, each holomorphic sectional curvature of $G_{i \bar{j}}$ is bounded below by -2 .
} 
or some truncation thereof. In all other cases the moduli space cannot be both smooth and complete for the kinetic-terms metric $G_{i j}$.

Working with non-complete and/or non-smooth Riemannian spaces is technically inconvenient. We try to improve the situation by smoothing out the singularities with some local surgery, i.e. by modifying the metric $G_{i j}$ in the vicinity of the "bad" points.

We stress that the modified metric is meant to be a mere technical trick to simplify the analysis of the geometry of the moduli space $\mathscr{M}$. However it is suggestive to phrase the surgery as it was an actual modification of the effective Lagrangian $\mathscr{L}_{\text {eff. }}$. The modification would be almost "harmless", since the original Lagrangian itself gave a poor description of the IR physics near the singularity in field space, so the local modification affects physical processes which were already outside the scope of $\mathscr{L}_{\text {eff. }}$. In a sense the locally modified Lagrangian is still a "good" effective Lagrangian, and should satisfy the swampland consistency conditions as far as they do not involve the region near the "bad" points in field space.

The allowed deformations of $\mathscr{L}_{\text {eff }}$ are quite restricted since they should preserve all gauge symmetries. In particular: (1) the deformation of the kinetic-terms metric should leave the discrete gauge group $\mathcal{G}$ as an exact symmetry of the problem; (2) the holonomy and isometry Lie algebras of $G_{i j}, \mathfrak{h o l}(\mathscr{M})$ and iso $(\mathscr{M})$, should be preserved.

Under the hypothesis that all singularities are fixed by a subgroup $\mathcal{G}_{*}$ of the neat group $\mathcal{G}$, it suffices to modify the metric on the universal cover $\widetilde{\mathscr{M}}$ in such a way that $\mathcal{G}$ is a freely acting group of isometries of the deformed metric. Since the Ricci curvature is expected to blow up at a fixed point of an unipotent isometry, a perturbation of the form

$$
G_{i j} \rightarrow G_{i j}^{\epsilon} \equiv G_{i j}+\epsilon R_{i j}, \quad \epsilon>0
$$

suggests itself. Except at loci where the curvature blows-up while the metric remains finite - which is exactly the characterization of finite-distance singularities - the correction to the metric is negligible for $\epsilon$ very small, so (2.14) is essentially a local modification of the geometry around the finite-distance singular loci. $\mathcal{G}$ is still an exact isometry of the perturbed metric, so we do not spoil the discrete gauge symmetry. More generally, the surgery (2.14) does not spoil any symmetry the original geometry may have. Condition (2) is also satisfied. ${ }^{10}$ We can see the modification (2.14) as the result of a backward Ricciflow [33] of the metric by the small time $t=-\epsilon / 2$, so that, heuristically, it looks like a RG flow to a slightly larger energy scale, in line with the physical interpretation of the finite-distance singularities as loci where new physics comes in. In view of eq. (2.8), replacing the WP metric $G_{j \bar{k}}$ by the Hodge one $K_{j \bar{k}}$ on a 3-CY moduli space amounts to the surgery (2.14) with $\epsilon=1 /(m+1)$; indeed our proposed prescription (2.14) is modelled on the standard math treatment of 3-CY complex moduli spaces.

\footnotetext{
${ }^{10}$ Let us check that the holonomy algebra $\mathfrak{h o l}(\mathscr{M})$ is preserved. We may assume $\widetilde{\mathscr{M}}$ to be irreducible without loss. If the Riemannian metric $G_{i j}$ is locally symmetric, it is Einstein, and the modification (2.14) is just a change of overall normalization of the metric by a factor $1+O(\epsilon)$. Otherwise $\mathfrak{h o l}(\mathscr{M})$ is one of the seven Berger holonomies [32]. For generic holonomy $\mathfrak{s o}(m)$ there is nothing to show. If $G_{i j}$ is Kähler, so is $G_{i j}^{\epsilon}$. For $\mathscr{M}$ a Calabi-Yau, hyperKähler, quaternionic-Kähler, $G_{2^{-}}$or $\operatorname{Spin}(7)$-manifold, $G_{i j}$ is either Ricci-flat or Einstein so the deformation (2.14) is either trivial or a slight rescaling of the metric.
} 
The modification (2.14) makes sense provided the Ricci curvature is bounded below, $R_{i j} \geq-K G_{i j}$, so that $G_{i j}^{\epsilon}$ is positive-definite for small $\epsilon$. The idea is that the modification replaces a small region around the singular point $x_{*}$ by a cusp of infinite length but finite volume $^{11}$ of order $O\left(\epsilon^{k}\right)$ for some $k>0$. When this happens, the singularity is pushed at infinite distance, and geodesic completeness is restored. Thus the volume conjecture still holds after the surgery, however the new "spurious" cusps are not associated to towers of light states as the genuine infinite ends of $\mathscr{M}$.

In this paper we assume that it is always possible to modify the metric locally at the singularities, while preserving $\mathcal{G}$ and $\mathfrak{h o l}(\mathscr{M})$, by replacing a neighborhood of the finitedistance singularity with a cusp of volume $O\left(\epsilon^{k}\right)$, so that the resulting Riemannian space is complete and smooth. All our arguments below are meant to apply to the "regularized" moduli manifold so constructed, which we shall denote simply $\mathscr{M}$. In the known examples the assumption holds true.

After the modification the geometric swampland conjectures still hold if they were satisfied by the original $\mathscr{L}_{\text {eff }}$, with the only exception that the distance conjecture does not apply to the spurious infinite ends introduced by blowing-up finite-distance singularities.

\subsection{Behavior at infinity}

In some argument below we need some more technical aspect of the geometry of $\mathscr{M}$ at infinite distance. In this sub-section we sketch the main issues; it may be omitted in a quick reading.

\subsubsection{Sign of curvature at infinity}

In their original paper [2] Ooguri and Vafa conjectured that the scalars' space $\mathscr{M}$ is noncompact of finite-volume. They also conjectured that the moduli-space scalar curvature $R$ is negative at infinity. In ref. [35] Trenner and Wilson constructed an explicit "counterexample" 12 to the last statement in the context of Type IIB on a certain 3-CY with $h^{2,1}=3$. In that example there is a real curve $C$ in moduli space, parametrized by $s \in \mathbb{R}$, such that, as we approach a "large complex structure limit" along this curve, the WP scalar curvature $R$ behaves as [35]

$$
R=\frac{32}{81} s^{3}+O\left(s^{2}\right) \quad \text { as } s \rightarrow+\infty \text { along } C,
$$

so in this limit $R$ is positive and unbounded. We wrote "counter-example" between quotes because this example does not contradict the physical picture of [2]. In the language of eq. (2.13), the physical intuition for Calabi-Yau moduli spaces goes roughly as follows: as we approach a generic point on the divisor $D_{\infty} \subset \overline{\mathscr{M}}$ (the infinite-distance locus) the Ricci curvature of the WP metric becomes negative hence bounded by (2.9), while as we approach a generic point on $D_{f}$ (finite-distance singularities) $R_{i j}$ becomes positive and divergent because of the contributions from loops of the finitely many additional light particles which can be computed in some "enlarged" effective field theory. What happens

\footnotetext{
${ }^{11}$ The basic reason of the finiteness of the volume is that we mod out the infinite group $\mathcal{G}_{*}$ which maps a small neighborhood of $x_{*}$ into itself.

${ }^{12}$ See theorem 3.2 of ref. [35].
} 
at the special points at infinity $D_{\infty} \cap D_{f}$ ? The obvious guess is that if we approach the intersection point following a curve $C$ along which

$$
m_{f} \rightarrow 0, \quad m_{\infty} \rightarrow 0 \quad \text { with } \quad \frac{m_{f}}{m_{\infty}} \rightarrow 0,
$$

where $m_{f}$ (resp. $m_{\infty}$ ) is the mass scales of the particles getting light along $D_{f}$ (resp. $D_{\infty}$ ), then the divergent positive contribution to $R$ from the finitely many massless particles along $D_{f}$ may win over the bounded negative contribution from the infinite tower of light states along $D_{\infty}$. This is what happens in the Trenner-Wilson example; along their curve $C m_{f}=O\left(s^{-2}\right)$ and $m_{\infty}=O\left(s^{-1}\right)$. We see that the non-positivity of $R$ at infinity has the same physical origin as the failure of the WP metric to be complete [23]. Then after replacing $G_{i \bar{j}}$ with its regularized version $G_{i \bar{j}}^{\epsilon}$, which it makes $\mathscr{M}$ into a complete manifold, we expect that also the problem with the sign of the scalar curvature at infinity is solved, that is, we expect that its scalar curvature $R^{\epsilon}$ to be negative and bounded (for fixed $\epsilon$ ) everywhere at infinity. Indeed, along $D_{\infty}$ the $\epsilon$-modification is inessential while along $D_{f}$ we have $G_{i \bar{j}}^{\epsilon} \approx \epsilon K_{i \bar{j}}$ so that from eq. (2.10) the scalar curvature is asymptotically negative and large (of order $O(1 / \epsilon)$ ). As a check we have computed (using Mathematica) the scalar curvature $R^{\epsilon}$ in the Trenner-Wilson example along the curve $C$ :

$$
R^{\epsilon}=-\frac{6}{\epsilon}\left(1+O\left(\epsilon^{2}\right)\right)+O(1 / s), \quad \text { as } s \rightarrow+\infty \text { along } C .
$$

In other words: the points on the curve $C$ for sufficiently large (but finite) $s$ are outside the region where the Lagrangian $\mathscr{L}_{\text {eff }}$ yields a reliable IR description of the physics, and the coupling $G_{i \bar{j}}$ (i.e. the WP metric) needs not to behave in a "physically reasonable" way at these points. Fix a regular point $p_{0} \in \mathscr{M}$; for all points at a sufficiently large distance from $p_{0}$ which are not too close to special loci where some "new physics" appears, $R$ is negative.

\subsubsection{Cusps}

We first consider the following simple but typical ${ }^{13}$ situation: $M$ is a complete non-compact Riemannian manifold and there is a compact subset $K \subset M$ such that $M \backslash K$ is the disjoint union of finitely many "ends at infinity" of $M$, the $\alpha$-th end $E_{\alpha}$ being diffeomorphic to $\mathbb{R} \times Z_{\alpha}$ for some connected manifold $Z_{\alpha}$, while the metric in $E_{\alpha}$ has the asymptotic form

$$
d s^{2} \equiv G_{i j} d x^{i} d x^{j} \approx d r^{2}+g(r, u)_{a b} d u^{a} d u^{b}, \quad \text { for } r \gg 1,
$$

where $r$ is the distance from some base point $* \in M, u^{a}$ are local coordinates in $Z_{\alpha}$, and $g(r, u)_{a b}$ is some $r$-dependent metric on $Z_{\alpha}$. Finiteness of the volume of $E_{\alpha}$ requires $\sqrt{\operatorname{det} g(r, u)}$ to decay more rapidly than $1 / r$ for large $r$. In the region where $(2.18)$ holds we have

$$
R_{r r} \approx-\partial_{r}^{2} \log \sqrt{\operatorname{det} g}-\frac{1}{4}\left\|\partial_{r} g\right\|^{2} .
$$

If $g(r, u)_{a b}$ goes to zero as slowly as a negative power of $r$, the r.h.s. is $O\left(1 / r^{2}\right)$ and $R_{r r} / g_{r r}$ is not bounded away from zero. On the other hand if $g(r, u)_{a b}$ goes to zero more rapidly

\footnotetext{
${ }^{13}$ Typical means, in particular, that this is the situation in all known explicit examples.
} 
than an exponential, say as $\approx C e^{-c r^{k}}$ with $k>1$ the curvature $R_{r r}$ is $O\left(r^{2(k-1)}\right)$ and unbounded below for large $r$. So, if the Ricci tensor is negative and bounded for large $r$, $g(r, u)_{a b}$ should be a sum of terms with exponential decay

$$
g(r, u)_{a b} \approx \sum_{i} e^{-c_{i} r} h^{(i)}(u)_{a b}
$$

Assuming the asymptotic metric to be enough regular, this leads to bounds for large $r$ of the form

$$
-K_{1} G_{i j} \leq R_{i j} \leq-K_{2} G_{i j} \quad \text { for large } r \text { along } E_{\alpha}
$$

for some constants $K_{1}, K_{2}>0$. We shall call a finite-volume end $E_{\alpha}$ with the behaviour (2.21) a "cusp". Prototypical examples are the cusps in an arithmetic quotient of a non-compact symmetric space ${ }^{14}$

$$
G(\mathbb{Z}) \backslash G(\mathbb{R}) / K
$$

Eq. (2.22) is the general form of the moduli space $\mathscr{M}$ when $\mathscr{L}_{\text {eff }}$ has a large supersymmetry (more than 8 supercharges). In these cases the nice geometry of the ends of the moduli space is directly related to the physics of quantum gravity as described by the distance conjecture [36]. In these extended susy examples the $U$-duality group $\mathcal{G} \equiv G(\mathbb{Z})$ acts faithfully (modulo finite groups) on the electro-magnetic charge lattice $V_{\mathbb{Z}}$, and each point at infinity $x_{\infty} \in \overline{\mathscr{M}}$ is fixed by a parabolic subgroup $\mathcal{G}_{x_{\infty}} \subset G(\mathbb{Z})$. States carrying electromagnetic charges $q \in V_{\mathbb{Z}}$ invariant under $\mathcal{G}_{x_{\infty}}$ have masses proportional to the length of the image in the Siegel domain of the shortest loop in $\mathscr{M}$ based at $x$ in the homotopy class of the elements of $\mathcal{G}_{x_{\infty}}$; since the map $\mu$ is a totally geodesic isometric embedding for $\mathcal{N} \geq 3$ the mass is also proportional to the length of the pre-image loop in $\mathscr{M}$, which for a good cusp is exponentially small, cfr. eq. (2.20). The same applies (with some subtlety) in the $\mathcal{N}=2$ case, using the relation between the kinetic terms of scalars and vectors implied by SUSY which replaces the totally geodesic embedding condition. Conjecturally this extends to the general quantum-consistent effective theory: the length of the loop in target space should be exponentially small to fit with the predictions of the distance conjecture. If the pre-image loop has a length which vanishes more rapidly than any exponential, say $O\left(e^{-c r^{k}}\right), k>1$, the derivative would be of order $e^{c r^{k}},\|d \mu\|^{2}=O\left(e^{3 c r^{k}}\right)$ which looks unreasonable.

Condition (*). Although the large $r$ behaviour (2.21) is expected for all quantum-consistent effective theories, to be very conservative in this paper we shall assume a much weaker condition on the large $r$ behaviour of the geometry after the smoothing surgery in section 2.3.3. First we assume that the Ricci curvature of $\mathscr{M}$ is still bounded below $R_{i j} \geq-K G_{i j}$. Since $\mathscr{M}$ is complete, for all $R>0$ there exists a Lipschitz continuous

\footnotetext{
${ }^{14}$ Here $G(\mathbb{R})$ is a non-compact real Lie group seen as a concrete group of matrices via a suitable representation of degree $\ell, K \subset G(\mathbb{R})$ is a maximal compact subgroup, and $G(\mathbb{Z}) \equiv G(\mathbb{R}) \cap \mathrm{GL}(\ell, \mathbb{Z})$ is the arithmetic subgroup consisting of matrices with integral entries. More generally, we may replace $G(\mathbb{Z})$ by a commensurable subgroup of $G(\mathbb{R})$.
} 
function $h_{R}: \mathscr{M} \rightarrow \mathbb{R}$ such that for some fixed constant $k>0[37]:{ }^{15}$

$$
0 \leq h_{R} \leq 1, \quad h_{R}=\left\{\begin{array}{ll}
1 & \text { for } r \leq R \\
0 & \text { for } r \geq 2 R,
\end{array} \quad\left|d h_{R}\right|<\frac{k}{R}\right.
$$

For typical asymptotic metrics of the form (2.18), (2.20) the Laplacian of $h_{R}$ is of order $O\left(R^{-1}\right)$ for large $R$. We shall require only the much weaker

Condition $((*))$. The Laplacian of $h_{R}$ is bounded by a constant $C$ independent of $R$

$$
\left|\Delta h_{R}\right|<C
$$

\section{OV manifolds}

In this section we assume that there exists a suitable local surgery, along the lines described in the previous section, such that the singularities of a suitable finite cover of the moduli space get repaired resulting in a smooth Riemannian manifold $\mathscr{M}$ which still satisfies the Ooguri-Vafa geometric swampland conjectures [2]. This certainly holds in the known examples of quantum consistent effective theories of gravity.

A smooth manifold which satisfies the Ooguri-Vafa geometric conditions, together with some mild "regularity" conditions, will be called an OV manifold. Understanding the geometry of OV manifolds and its physical implications is one of the themes of this paper.

We propose the following definition of OV manifold:

Definition 1. The point is a zero-dimensional OV space. In positive dimension an $O V$ manifold is a connected, complete, Riemannian manifold $M$ with a smooth simplyconnected cover $\widetilde{M}$ which has a de Rham decomposition of the form

$$
\widetilde{M}=F \times \widetilde{M}_{1} \times \cdots \times \widetilde{M}_{s}
$$

and a smooth finite cover of the form

$$
M^{b}=F \times \widetilde{M}_{1} / \mathcal{G}_{1} \times \cdots \times \widetilde{M}_{s} / \mathcal{G}_{s}, \quad \mathcal{G}_{k} \subset \operatorname{Iso}\left(\widetilde{M}_{k}\right)
$$

such that:

OV0. The flat factor $F$ is either trivial or the real line $\mathbb{R}$;

OV1. $M_{k} \equiv \widetilde{M}_{k} / \mathcal{G}_{k}$ is a complete, irreducible, non-compact manifold of finite volume;

OV2. $\widetilde{M}_{k}$ is diffeomorphic to $\mathbb{R}^{m_{k}}$ and $\mathcal{G}_{k} \cong \pi_{1}\left(M_{k}\right)$ is a torsion-less discrete group of isometries of $\widetilde{M}_{k}$ generated by elements $\left\{u_{i}\right\}$ such that

$$
\inf _{x \in \widetilde{M}_{k}} d\left(u_{i} x, x\right)=0 .
$$

\footnotetext{
${ }^{15}$ For instance, we can choose $h_{R}=\varphi(r / R)$ where $\varphi$ is a smooth function on the real line with $0 \leq \varphi \leq 1$, $\varphi=1$ for $x \leq 1$ and $\varphi=2$ for $x \geq 2$.
} 
OV3. The Ricci curvature $R_{i j}^{(k)}$ of $M_{k}$ is bounded below by a negative constant

$$
R_{i j}^{(k)} \geq-K_{k} g_{i j}^{(k)}, \quad K_{k}>0,
$$

and condition $(*)$ (eq. (2.24)) is satisfied.

Some comments on the definition are in order:

- OV1 is the volume conjecture [2] and OV2 is a refined version of the $\pi_{1}$ conjecture. A stronger version of $\mathbf{O V 2}$, which holds in all known examples, would be:

OV2 $* \mathcal{G}_{k}$ is isomorphic to a strongly approximant ${ }^{16}$ subgroup $\stackrel{\mathscr{G}}{k}_{k}$ of an arithmetic group $G(\mathbb{Z}) \subset \operatorname{GL}(n, \mathbb{Z}) . \stackrel{\mathscr{\mathcal { G }}}{k}_{k}$ is required to be neat, semi-simple, and to have a finiteindex subgroup generated by finitely-many unipotent elements $\left\{u_{i}\right\} \subset \stackrel{\circ}{\mathcal{G}}_{k} \cong \mathcal{G}_{k}$ which satisfy eq. (3.3).

- the point and the real line $\mathbb{R}$ are OV manifolds. This is required by math elegance and is consistent since all the 'magic' properties of OV manifolds are shared by the point and $\mathbb{R}$. The point and $\mathbb{R}$ do appear as moduli of consistent gravities, and even as their factor spaces: think of M-theory on $\mathbb{R}^{10-k, 1} \times S^{k}$ for $k=0,1,2$; in particular for $k=2 \mathscr{M}=\mathbb{R} \times(\mathrm{SL}(2, \mathbb{Z}) \backslash \mathrm{SL}(2, \mathbb{R}) / \mathrm{U}(1))$.

- OV3 is a milder version of the "regularity" conditions discussed in section 2.4.2 related to the distance conjecture and the expected behaviour at infinity.

Remark 3. The emergence proposal (section 5 of [4]) gives a model-independent bridge between the distance conjecture and the geometry of $\mathscr{M}$ at infinite distance. One starts with a compactification $\overline{\mathscr{M}}$ of $\mathscr{M}$; the metric is singular along the loci in $\overline{\mathscr{M}}$ where more degrees of freedom get massless: the singular contributions to the metric come from loops of light states. When an infinite tower of d.o.f. get massless the divergence of the metric is so severe that the locus is pushed at infinite distance. The singular part of the metric due to a infinite tower of light states is universal, and can be read from any example. Thus one gets back the strong version of OV3 inferred from examples in section 2.4.2: there exists a $h_{R}$ as in (2.23) with $\left|\Delta h_{R}\right| \lesssim c / R$. We opted for the much weaker version $\left|\Delta h_{R}\right|<c^{\prime}$ : we see OV3 as a mere technical condition, not an additional swampland conjecture, and our OV3 is just the weakest assumption required to prove the "magical" properties of the OV manifolds.

\subsection{First consequences of the definition}

\subsubsection{Holonomy groups and the graded algebra $\mathcal{P}^{\bullet}$}

A non-compact, complete manifold of non-negative Ricci curvature cannot have finite volume ${ }^{17}$, so the Ricci curvature of the irreducible factor spaces $\widetilde{M}_{k}$ somewhere should have

\footnotetext{
${ }^{16}$ We recall the definition of "strong approximant" subgroup. We see the arithmetic group $G(\mathbb{Z})$ as a concrete group of integral matrices, i.e. it comes with a preferred embedding $G(\mathbb{Z}) \subset \mathrm{GL}(n, \mathbb{Z})$ for some $n$. For $p$ a prime, we write $G(\mathbb{Z} / p \mathbb{Z})$ for the finite group of Lie type obtained by reducing the matrices mod $p$. We have the canonical surjection $G(\mathbb{Z}) \stackrel{\pi_{p}}{\longrightarrow} G(\mathbb{Z} / p \mathbb{Z}) . \gamma: \Gamma \hookrightarrow G(\mathbb{Z})$ is said to be a strong approximant iff the group homomorphism $\pi_{p} \circ \gamma: \Gamma \rightarrow G(\mathbb{Z} / p \mathbb{Z})$ is surjective for almost all primes $p$.

${ }^{17}$ This follows from the Calabi-Yau lower bound on the volume $[37,38]$ see theorem 7 and appendix iii in ref. [37].
} 
some strictly negative eigenvalue. This rules out a few Riemannian geometries:

\begin{tabular}{cc|c}
\hline allowed $\widetilde{M}_{k}$ & $\mathfrak{h o l}\left(\widetilde{M}_{k}\right)$ & ruled out \\
\hline strictly generic holonomy & $\mathfrak{s o}\left(m_{k}\right)$ & Calabi-Yau \\
strictly Kähler & $\mathfrak{u}\left(m_{k} / 2\right)$ & hyperKähler \\
strict negative quaternionic-Kähler & $\mathfrak{s p}(1) \oplus \mathfrak{s p}\left(m_{k} / 4\right)$ & positive quaternionic-Kähler \\
non-compact symmetric spaces $G / K$ & $\mathfrak{k}$ & $G_{2}$ and Spin(7) manifolds \\
\hline
\end{tabular}

It is remarkable that, a part for "strictly generic" (which, roughly, corresponds to the non-SUSY case), the list of allowed geometries is reminiscent of the list of target spaces for supergravity with more than 2 supercharges. We recall that the holonomy algebra $\mathfrak{h o l}(\mathscr{M})$ of the scalars' manifold $\mathscr{M}$ of a supergravity is determined as follows: from its SUSY algebra and super-multiplet content we read the R-symmetry Lie algebra $\mathfrak{r}$ and its representation $\sigma$ on the scalar fields; $\mathfrak{h o l}(\mathscr{M})$ then has the form [36] (here $\left.m=\operatorname{dim}_{\mathbb{R}} \mathscr{M}\right)$

$$
\sigma(\mathfrak{r}) \subseteq \mathfrak{h o r}(\mathscr{M}) \subseteq \sigma(\mathfrak{r})+\mathfrak{z}(\sigma(\mathfrak{r})) \subset \mathfrak{s o}(m) \quad \mathfrak{z}(\sigma(\mathfrak{r})) \stackrel{\text { def }}{=} \text { centralizer of } \mathfrak{r} \text { in } \mathfrak{s o}(m)
$$

In SUGRA the R-symmetry is a gauge symmetry with composite gauge fields $\partial_{\mu} \phi^{i} \Omega(\phi)_{i b}^{a}$, where $\Omega(\phi)_{i b}^{a}$ is the projection of the Levi-Civita connection of $G(\phi)_{i j}$ on the sub-algebra $\sigma(\mathfrak{r})$.

Let $\mathfrak{h}_{k} \equiv \mathfrak{h o r}\left(\widetilde{M}_{k}\right) \subset \mathfrak{s o}\left(m_{k}\right)$ be the irreducible holonomy algebra of the $k$-th factor space $\widetilde{M}_{k}$. The graded algebra $\mathcal{P}_{k}^{\bullet}$ of parallel forms on $\widetilde{M}_{k}$ is ${ }^{18}$

$$
\mathcal{P}_{k}^{\bullet} \equiv \bigoplus_{j=0}^{m_{k}} \mathcal{P}_{k}^{j} \cong \bigoplus_{j=0}^{m_{k}}\left(\wedge^{j} \mathbb{R}^{m_{k}}\right)^{\mathfrak{h}_{k}} .
$$

When $\mathfrak{h}_{k}=\mathfrak{s o}\left(m_{k}\right), \mathcal{P}_{k}^{\bullet}$ is spanned by 1 and a volume form $\varepsilon$ : we say that $\mathcal{P}_{k}^{\bullet}$ is trivial. When $\widetilde{M}_{k}$ is strictly Kähler, $\mathcal{P}_{k}^{\bullet}=\mathbb{R}[\omega] / \omega^{1+m_{k} / 2}$ with $\omega$ the Kähler form. When $\widetilde{M}_{k}$ is strictly quaternionic-Kähler $\mathcal{P}_{k}^{\bullet}=\mathbb{R}[\Omega] / \Omega^{1+m_{k} / 4}$ with $\Omega$ the canonical 4-form. When $\widetilde{M}_{k}$ is a symmetric manifold the algebra $\mathcal{P}_{k}^{\bullet}$ is typically larger; but there are two exceptions: $\mathrm{SO}(n, 1) / \mathrm{SO}(n)$ which has generic holonomy $\mathfrak{s o}(n)$, and $\mathrm{SU}(m, 1) / \mathrm{U}(m)$ which has strict Kähler holonomy $\mathfrak{u}(m)$. We stress that the surgery of section 2.3.3 preserves the algebra $\mathcal{P}^{\bullet}$.

The generic OV manifold has no non-trivial parallel form. Let us consider an example in the other extremum, where $\mathcal{P}^{\bullet}$ is so rich that fully determines the metric (up to overall normalization).

Example 1. Suppose the irreducible OV manifold $\mathscr{M}$ has R-algebra $\mathfrak{r}=\mathfrak{s u}(8)$ and Rrepresentation $\sigma$ the $\mathbf{7 0}$. Then - up to finite covers $-\mathscr{M}$ is globally isometric to the locally symmetric space

$$
E_{7}(\mathbb{Z}) \backslash E_{7}(\mathbb{R}) / \mathrm{SU}(8)
$$

\footnotetext{
${ }^{18}$ If $V$ is a vector space carrying a representation of the reductive Lie algebra $\mathfrak{h}$, the symbol $V^{\mathfrak{h}}$ denotes the $\mathfrak{h}$-invariant subspace of $V$, that is, the subspace on which $\mathfrak{h}$ acts trivially.
} 
where $E_{7}(\mathbb{Z})$ denotes a maximal arithmetic subgroup of $E_{7}(\mathbb{R})$ (the split real form of $E_{7}$ ). ${ }^{19}$ This is the moduli space of Type II compactified on $T^{6}$ with the correct $U$-duality group $\mathcal{G}=E_{7}(\mathbb{Z})$ (up to commensurability).

\subsubsection{Liouville property}

Many nice properties of supersymmetric field theories (with or without gravity), such as the non-renormalization/rigidity theorems, may be traced back to the fact that their moduli spaces $\mathscr{M}$ are "Liouvillic". This property holds automatically for OV manifolds. Morally speaking, "most" of the good aspects of SUSY appear to be given for free once the swampland conjectures are satisfied.

Definition 2. The manifold $M$ is Liouvillic for the class $\mathcal{C}$ of functions $f: M \rightarrow \mathbb{R}$ if

$$
f \in \mathcal{C} \text { and }|f|<K<\infty \Rightarrow f=\text { constant. }
$$

Proposition 1. Irreducible OV manifolds $M$ are Liouvillic for the sub-harmonic functions,

$$
\text { i.e. } \quad \Delta f \geq 0 \text { and }|f|<K<\infty \Rightarrow f=\text { constant. }
$$

Proof. For $M \equiv \mathbb{R}$ this reduces to the well-known fact that a bounded convex function $f: \mathbb{R} \rightarrow \mathbb{R}$ is a constant. For $M$ a complete, non-compact manifold of finite volume, we write $u \equiv f+K . u$ is a non-negative function bounded above by $2 K$, and for all $p>1$

$$
\int_{\mathscr{M}} u^{p} d \mathrm{vol} \leq(2 K)^{p} \cdot \operatorname{Vol}(\mathscr{M})<\infty
$$

and then $u$ is a constant by theorem 3 in [37].

The statements holds even for reducible OV manifolds as long as they have no flat factor in eq. (3.2). In particular, it holds for all 4d SUGRA satisfying the Ooguri-Vafa swampland conjectures [2]. The Liouville property seems to be crucial for the swampland story: this is the case for all supersymmetric consistent effective theories.

Remark 4. Standard Seiberg-Witten theory $[42,43]$ is grounded on the fact that the Coulomb branch of an UV complete $\mathcal{N}=2$ QFTs satisfies the much weaker property of being Liouvillic for the sub-pluriharmonic functions.

\section{The gauge couplings $\tau(\phi)_{a b}$ as a map}

One wishes to understand which gauge coupling $\tau(\phi)_{a b}$ may appear in a consistent effective Lagrangian (2.1) of quantum gravity. These couplings have a natural geometric description which cries for an intrinsic characterization of the allowed $\tau(\phi)_{a b}$.

\footnotetext{
${ }^{19}$ With respect to some structure of $E_{7}(\mathbb{R})$ as an algebraic group defined over $\mathbb{Q}$. The $\mathbb{Q}$-algebraic structure is determined by quantum consistency: in this case $E_{7}$ is identified with the universal Chevalley group of type $E_{7}$ (a scheme over $\mathbb{Z}$ ) and $E_{7}(\mathbb{Z})$ is the groups of its points valued in $\mathbb{Z}$.
} 


\subsection{Generalities}

For fixed values of the scalar fields $\phi^{i}$ (and given duality-frame), the gauge coupling $\tau(\phi)_{a b}$ is an element of Siegel's upper half-space

$$
\boldsymbol{H}_{h} \equiv\left\{\tau \in \operatorname{Mat}_{h \times h}(\mathbb{C}) \mid \tau=\tau^{t}, \operatorname{Im} \tau>0\right\} \cong \operatorname{Sp}(2 h, \mathbb{R}) / \mathrm{U}(h) .
$$

$\boldsymbol{H}_{h}$ is a non-compact Kähler symmetric space on which the group $\operatorname{Sp}(2 h, \mathbb{R})$ acts transitively by isometries. Its maximal arithmetic subgroup $\operatorname{Sp}(2 h, \mathbb{Z}) \subset \operatorname{Sp}(2 h, \mathbb{R})$ is the group of electro-magnetic duality-frame rotations. As a Riemannian space $\boldsymbol{H}_{h}$ is Hadamard ${ }^{20}$ [39, 40] so diffeomorphic to $\mathbb{R}^{h(h+1)}$. As a complex manifold $\boldsymbol{H}_{h}$ is biholomorphic to the (symmetric) bounded domain in $\mathbb{C}^{h(h+1) / 2}$ of symmetric $h \times h$ matrices $Z$ with $1-Z \bar{Z}>0$.

In general the complex gauge couplings $\tau(\phi)_{a b}$ are not well-defined functions on the scalars' space $\mathscr{M} \equiv \widetilde{\mathscr{M}} / \mathcal{G}$. Indeed, when we go around a non-contractible loop $\gamma$ in $\mathscr{M}$, we may come back and find that the duality frame was rotated by a non-trivial element of $\operatorname{Sp}(2 h, \mathbb{Z})$. This yields a monodromy representation

$$
\rho: \pi_{1}(\mathscr{M}) \equiv \mathcal{G} \rightarrow \operatorname{Sp}(2 h, \mathbb{Z}) .
$$

The image $\rho\left(\pi_{1}(\mathscr{M})\right) \subset \operatorname{Sp}(2 h, \mathbb{Z})$ is the monodromy group $\Gamma$, cfr. eq. (2.4).

To get actual coupling functions, it is convenient to lift the gauge couplings to the smooth universal cover $\widetilde{\mathscr{M}}$; then we may identify them with the map

$$
\widetilde{\mu}: \widetilde{\mathscr{M}} \rightarrow \operatorname{Sp}(2 h, \mathbb{R}) / \mathrm{U}(h) \equiv \boldsymbol{H}_{h}, \quad \widetilde{\mu}: \phi \mapsto \tau(\phi)_{a b},
$$

which lifts the intrinsic gauge coupling map $\mu,{ }^{21}$

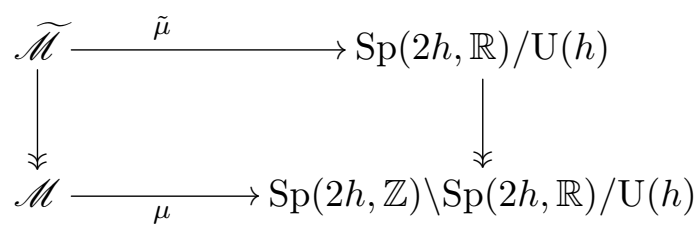

One also says that the lifted map $\tilde{\mu}$ is twisted by the monodromy representation $\rho$, i.e. $\tilde{\mu}$ satisfies the functional equations

$$
\tilde{\mu}(g \cdot x)=\rho(g) \cdot \tilde{\mu}(x), \quad \forall g \in \mathcal{G} \subset \operatorname{Iso}(\widetilde{\mathscr{M}}), \quad x \in \widetilde{\mathscr{M}} .
$$

The target space of $\mu$ in (4.4), $\operatorname{Sp}(2 h, \mathbb{Z}) \backslash \boldsymbol{H}_{h}$, is an irreducible, non-compact, locally symmetric space of the special form $G_{\mathbb{Z}} \backslash G / K$ where $G_{\mathbb{Z}} \subset G$ is an arithmetic (hence Zariski-dense [22]) discrete subgroup. ${ }^{22}$ Maps between spaces with these special properties are well studied in mathematics.

\footnotetext{
${ }^{20}$ That is, a simply-connected manifold with non-positive sectional curvatures; in facts $\boldsymbol{H}_{h}$ has even non-positive curvature operators.

${ }^{21}$ Throughout the paper double-headed arrows stand for canonical projections.

${ }^{22}$ With respect to the obvious structure of $G \equiv \mathrm{Sp}(2 h, \mathbb{R})$ as an algebraic group over $\mathbb{R}$. In facts, $\operatorname{Sp}(2 h, \mathbb{Z}) \subset \operatorname{Sp}(2 h, \mathbb{R})$ is a maximal arithmetic subgroup [41]. In the present context it is more natural to consider $G$ as the real locus of an algebraic group defined over $\mathbb{Q}$, see [15] and references therein.
} 
From an algebro-geometric viewpoint the target space $\operatorname{Sp}(2 h, \mathbb{Z}) \backslash \boldsymbol{H}_{h}$ is a normal quasiprojective variety [44, 45], called the Siegel variety (or Siegel scheme), a special instance of Shimura variety $[46,47] . \operatorname{Sp}(2 h, \mathbb{Z}) \backslash \boldsymbol{H}_{h}$ is not smooth since $\operatorname{Sp}(2 h, \mathbb{Z}) /\{ \pm 1\}$ does not act freely on $\boldsymbol{H}_{h}$. This can be easily repaired by replacing $\operatorname{Sp}(2 h, \mathbb{Z})$ with a neat finite-index subgroup $\Lambda_{h} \subset \operatorname{Sp}(2 h, \mathbb{Z})$ [22]. Then $\Lambda_{h} \backslash \boldsymbol{H}_{h}$ is a smooth quasi-projective finite cover of $\operatorname{Sp}(2 h, \mathbb{Z}) \backslash \boldsymbol{H}_{h} . \quad \Lambda_{h} \backslash \boldsymbol{H}_{h}$ is a non-compact manifold of finite volume whose fundamental group $\Lambda_{h}$ is generated by unipotent elements: $\Lambda_{h} \backslash \boldsymbol{H}_{h}$ is a basic example of irreducible OV manifold. $\Lambda_{h} \backslash \boldsymbol{H}_{h}$ has a canonical compactification to a normal projective variety, the Baily-Borel compactification ${\overline{\Lambda_{h} \backslash \boldsymbol{H}_{h}}}^{B B}$ [44, 45]. ${\overline{\Lambda_{h} \backslash \boldsymbol{H}_{h}}}^{B B}$ is not smooth for $h>1$. However we may blow-up it into a smooth projective variety $\boldsymbol{Y}_{h}$ such that the divisor at infinity $\boldsymbol{Y}_{h} \backslash\left(\Lambda_{h} \backslash \boldsymbol{H}_{h}\right)$ is simple normal crossing. This can be done rather explicitly in terms of a suitable toroidal compactification of $\Lambda_{h} \backslash \boldsymbol{H}_{h}$ [48-50].

Then, replacing $\mathscr{M}$ by a finite cover (if necessary), we see the gauge coupling $\mu$ as a map between smooth OV manifolds

$$
\mu: \mathscr{M} \rightarrow \Lambda_{h} \backslash \boldsymbol{H}_{h} .
$$

It is remarkable that both the source and target spaces of $\mu$ are OV manifolds. In all known examples of quantum-consistent theories (such as compactifications of the superstring on Calabi-Yau's) both the image $\mu(\mathscr{M}) \hookrightarrow \Lambda_{h} \backslash \boldsymbol{H}_{h}$ and the fibers $\mu^{-1}(s) \hookrightarrow \mathscr{M}$ (equipped with the Riemannian structure induced by their respective embedding $\left.{ }^{23}\right)$ are also OV spaces. ${ }^{24}$ This is likely to remain true for all effective theories of quantum-consistent gravity.

One goal of this note is to give a preliminary discussion of the following

Question. What properties should have the gauge coupling map $\mu$ in eq. (4.6) for the Lagrangian (2.1) not to sink in the swampland?

If a simple condition on $\mu$ exists at all, it should be invariant under $\operatorname{Sp}(2 h, \mathbb{Z})$ rotations of the electro-magnetic frame. Since we consider only the very extreme IR limit, this means that the property should be invariant for $\operatorname{Sp}(2 h, \mathbb{R})$.

\subsection{Three different viewpoints on $\tau(\phi)_{a b}$}

Since the coupling $\tau(\phi)_{a b}$ is multi-valued in $\mathscr{M},{ }^{25}$ its value in a given vacuum $\phi$ is not an intrinsic observable, and we should replace it with some invariantly-defined quantity.

Automorphic viewpoint. In the case of a single photon $h=1$, the modular curve $\operatorname{SL}(2, \mathbb{Z}) \backslash \boldsymbol{H}_{1}$ is biholomorphic to the punctured sphere

$$
j: \overline{\mathrm{SL}(2, \mathbb{Z}) \backslash \boldsymbol{H}_{1}} \Im \mathbb{P}^{1}
$$

\footnotetext{
${ }^{23}$ In these examples $\mu$ is an embedding not just an immersion.

${ }^{24}$ The statement holds without exceptions because we defined the point to be an OV manifold.

${ }^{25}$ In the SUSY case (and also in the non-SUSY one under the naturalness condition we propose in section 7), $\tau(\phi)_{a b}$ must be multi-valued unless it is a numerical constant as in the examples discussed in [51].
} 
and one may use as the intrinsically-defined gauge coupling the value of the Hauptmodul $j$ which is independent of the branch of the multi-valued function $\tau$ (here $q \equiv e^{2 \pi i \tau}$ )

$$
j(\tau) \equiv \frac{\left(\theta_{2}(q)^{8}+\theta_{3}(q)^{8}+\theta_{4}(q)^{8}\right)^{3}}{13824 \eta(q)^{24}}=\frac{1}{q}+744+196884 q+\cdots
$$

This is the viewpoint taken in [51]. In principle this strategy may be applied for all $h$ since, by the already mentioned Baily-Borel theorem [44], $\Lambda_{h} \backslash \boldsymbol{H}_{h}$ has enough automorphic invariant functions to fully characterize its points.

Total space viewpoint. The space $\Lambda_{h} \backslash \boldsymbol{H}_{h}\left(\right.$ resp. $\left.\boldsymbol{H}_{h}\right)$ is the moduli space of enhanced ${ }^{26}$ principally polarized Abelian varieties over $\mathbb{C}$ of dimension $h$ (resp. marked, principally polarized, Abelian varieties). As for the axion-dilaton $\tau \equiv C_{0}+i e^{-\Phi}$ in $F$-theory [52], often it is more convenient to think of the gauge couplings as a fibration $\varpi: \mathscr{X} \rightarrow \mathscr{M}$ whose fibers $\mathscr{X}_{\phi}$ are (enhanced) principally polarized Abelian varieties of periods $\tau(\phi)_{a b}$, and then describe the properties of the gauge couplings in terms of the intrinsic geometry of the total space $\mathscr{X}$ of the fibration.

There is a more physical "total space" construction. One compactifies the $4 \mathrm{~d}$ effective theory down to $3 \mathrm{~d}$ on a circle; each $4 \mathrm{~d}$ vector field yields two new scalars in $3 \mathrm{~d}$ and we get two additional scalars from the metric by the KK mechanism. The resulting 3d scalars' manifold $\mathscr{M}_{3}$ is fibered over the 4 d scalars' manifold $\mathscr{M}$, and the gauge coupling map $\mu$ is encoded in the fiber's geometry (see section 7.2.4 for details). So the two $4 \mathrm{~d}$ couplings $G(\phi)_{i j}$ and $\mu(\phi)_{a b}$ get geometrically unified in the intrinsic Riemannian geometry of the 3d target manifold $\mathscr{M}_{3}$ which, according to the swampland conjectures, should also be an Ooguri-Vafa space with its own discrete gauge group $\mathcal{G}_{3} \triangleright \mathcal{G} \sim \mathcal{G}^{0} \times \Gamma$. We shall use this "total space" viewpoint when convenient.

Elementary viewpoint. We mostly adopt a more naive strategy: instead of considering subtle automorphic invariants of the gauge couplings, we shall see (4.6) as a mere smooth map between Riemannian manifolds, and rely on the invariants of smooth maps which are defined in Differential Geometry (DG) textbooks.

\subsection{DG invariants of the gauge couplings $\tau(\phi)_{a b}$}

The basic DG invariants of a smooth map $\phi: X \rightarrow Y$ between Riemann manifolds (with metrics $G_{i j}$ and $h_{a b}$, respectively) are:

- its energy $E[\phi]$ given by the value of the Dirichlet integral

$$
E[\phi]=\frac{1}{2} \int_{X} d^{n} x \sqrt{\operatorname{det} G} G^{i j} h_{a b} \partial_{i} \phi^{a} \partial_{j} \partial^{b} \phi^{a},
$$

that is, the action of the Euclidean $\sigma$-model with target $Y$ and source space-time $X$;

\footnotetext{
${ }^{26}$ The Siegel variety $\operatorname{Sp}(2 h, \mathbb{Z}) \backslash \boldsymbol{H}_{h}$ is the moduli space of principally polarized Abelian varieties. Going to the smooth covering Shimura variety $\Lambda_{h} \backslash \boldsymbol{H}_{h}$ leads to the moduli of polarized Abelian varieties endowed with some extra structure: e.g. if $\Lambda_{h}$ is the kernel of $\operatorname{Sp}(2 h, \mathbb{Z}) \rightarrow \operatorname{Sp}(2 h, \mathbb{Z} / m \mathbb{Z})(m \geq 3)$ the extra structure is a choice of generators of the group of $m$-torsion points. An Abelian variety with such extra structure is called an enhanced Abelian variety.
} 
- its tension field on $X$

$$
T(x)^{a} \stackrel{\text { def }}{=}-h(\phi(x))^{a b} \frac{\delta E[\phi]}{\delta \phi^{b}(x)} \equiv D^{i} \partial_{i} \phi^{a}(x) \in C^{\infty}\left(X, \phi^{*} T Y\right) .
$$

We replace our original question in section 4.1 with a less ambitious one:

Simpler question. What can we say about the $D G$ invariants $E[\mu]$ and $T[\mu]$ of the gauge coupling map $\mu: \mathscr{M} \rightarrow \Lambda_{h} \backslash \boldsymbol{H}_{h}$ in a quantum-consistent $4 d$ effective theory of gravity?

A partial answer will be given in section 6.3.

Although these DG invariants have a simple definition, in quantum-consistent effective theories of gravity they seem to involve rather deep number-theoretical issues. For instance, the allowed energy levels $E[\mu]$ of the gauge couplings $\mu$ in a quantum-consistent effective theory of gravity belong to a certain discrete subset $\Xi \subset \mathbb{R}_{\geq 0}$, the gauge couplings' energy spectrum, which carries a number-theoretic fragrance. Computing $\Xi$ is very hard except in some extremely simple class of effective models.

Extremely simple situation. The simplest possible quantum-consistent $4 \mathrm{~d}$ effective theories are the ones with the properties:

(1) the effective theory has $\mathcal{N} \geq 2$ local supersymmetry, and (2) its $U$-duality group $\mathcal{G}$ is commensurable to the group $\boldsymbol{G}(\mathbb{Z})$ of " $\mathbb{Z}$ valued" points in a universal Chevalley group-scheme $\boldsymbol{G}$ without simple factors of type $C_{h}$.

The prototypical $4 \mathrm{~d}$ effective theory satisfying ( $\$)$ is obtained by compactifying the $10 \mathrm{~d}$ Type II superstring on a flat 6 -torus $T^{6}$ (see appendix of [53]): in this case $\mathcal{N}=8$ and $\boldsymbol{G}$ has type $E_{7}$ (cfr. example 1$)$. Whenever $(\$)$ holds one has (for a standard normalization of the metrics)

$$
\Xi=\mathcal{I} \cdot \frac{m}{2 \operatorname{Vol}(K)} \prod_{\ell=1}^{r} \zeta\left(d_{\ell}\right)
$$

where $\left\{d_{\ell}\right\}$ are the degrees of the independent Casimir invariants of the real Lie group $\boldsymbol{G}(\mathbb{R}), K \subset G(\mathbb{R})$ is a maximal compact subgroup, ${ }^{27} \mathrm{Vol}(K)$ its volume (computed by the Macdonald formula [54]); $\zeta(s)$ is the Riemann $\zeta$-function, $m \equiv \operatorname{dim} G-\operatorname{dim} K$, and $\mathcal{I} \subset \mathbb{N}$ is the set of indices of finite-index subgroups of the maximal arithmetic group $\boldsymbol{G}(\mathbb{Z})$. If the $U$-duality group is precisely $\mathcal{G}$, the energy of the coupling constants is given by the r.h.s. of (4.11) with $\mathcal{I}$ replaced by $[\boldsymbol{G}(\mathbb{Z}): \mathcal{G}]$. Eq. (4.11) follows from standard susY arguments together with the Langlands volume formula for arithmetic quotients [55]. In the case of Type II on $T^{6}$ all degrees $d_{\ell}$ are even, so the energy of the gauge coupling has a closed expression in terms of Bernoulli numbers: $E(\mu)$ is a know rational number times $\pi^{35}$. For Type II on $T^{5}$, a part for a power of $\pi$, the energy has a transcendental factor $\zeta(5) \zeta(9)$.

\footnotetext{
${ }^{27}$ One can show that the absolute ranks of the two Lie groups $G(\mathbb{R})$ and $K$ are equal.
} 


\subsection{Tension field and harmonic maps}

To answer the simpler question, we start by recalling some basic facts about energies and tensions of smooth maps. Building on these facts, in the next section we shall introduce a more detailed and elegant structure, that we call domestic geometry, modelled on the variations of Hodge structures (VHS) [56-61] and the more general $t t^{*}$ geometry [25, 62-65].

Let $(X, G)$ and $(Y, h)$ be two Riemannian manifolds. As already mentioned, the energy $E(\phi)$ of a map $\phi: X \rightarrow Y$ is the action of the Euclidean $\sigma$-model with target space $Y$ and space-time $X$, see eq. (4.9). The map $\phi$ is harmonic iff it is a solutions of the corresponding equations of motion ${ }^{28}$

$$
0=T[\phi]^{a} \stackrel{\text { def }}{=} G^{i j} D_{i} \partial_{j} \phi^{a} \equiv G^{i j}\left(\partial_{i} \partial_{j} \phi^{a}-\gamma_{i j}^{k} \partial_{k} \phi^{a}+\Gamma_{b c}^{a} \partial_{i} \phi^{b} \partial_{j} \phi^{c}\right)
$$

that is, if its tension $T[\phi] \in \phi^{*} T Y$ vanishes. A finite-energy harmonic map is simply an instanton of the $\sigma$-model (4.9), i.e. a classical Euclidean-signature solution of finite action.

When $X$ has dimension 1, a harmonic map is just a geodesic on $Y$ of constant velocity. More generally, a map $\phi$ is totally geodesic iff the full matrix $D_{i} \partial_{j} \phi^{a}$ vanishes and not just its trace as for a general harmonic map.

When the source space $X$ is Kähler, eq. (4.12) reduces to

$$
0=G^{i \bar{k}} D_{i} \partial_{\bar{k}} \phi^{a} \equiv G^{i \bar{k}}\left(\delta_{b}^{a} \partial_{i}+\Gamma_{b c}^{a} \partial_{i} \phi^{c}\right) \partial_{\bar{k}} \phi^{b}
$$

and all dependence on the source-space Christoffel symbols $\gamma_{i j}^{k}$ drops out. In this situation the map $\phi$ is said to be pluri-harmonic if the full type- $(1,1)$ tensor $D_{i} \partial_{\bar{k}} \phi^{a}$ vanishes and not just its trace. This condition depends only on the complex structure of $X$, and is independent of the specific Kähler metric $G_{i \bar{k}}$. This means that a pluri-harmonic map is a classical solution of the $\sigma$-model for all choices of the source metric $G_{i \bar{k}}$ as long as it is Kähler. In particular $\phi$ remains a solution if we repair the singularities in the Kähler metric $G_{i \bar{k}}$ by a local surgery which keeps it Kähler as in section 2.3.3. If, in addition, the target space $Y$ is also Kähler with complex coordinates $z^{a}$, the pluri-harmonic condition reduces to $D_{i} \partial_{\bar{k}} z^{a}=0$ which is automatically satisfied if the stronger condition $\partial_{\bar{k}} z^{a}=0$ holds, i.e. if the map $z: X \rightarrow Y$ is holomorphic.

A useful fact is that the composition $\iota \circ \phi: X \rightarrow Z$ of a harmonic map $\phi: X \rightarrow Y$ and a totally geodesic map $\iota: Y \rightarrow Z$ is also harmonic [66].

\subsubsection{Energy and tension of maps into symmetric spaces}

The last observation allows us to describe in a simple way the harmonic maps from a Riemannian manifold $M$ to a symmetric space $G / H$. The map of main interest is the covering gauge coupling $\tilde{\mu}$ in eq. (4.3), and we write explicit expressions for $G / H=\operatorname{Sp}(2 h, \mathbb{R}) / \mathrm{U}(h)$, the general case then being obvious. One considers Cartan's totally geodesic embed-

\footnotetext{
${ }^{28}$ In eq. (4.12) $\gamma_{i j}^{k}$ and $\Gamma_{b c}^{a}$ are the Christoffel symbols for, respectively, the metric $G_{i j}$ and $h_{a b}$.
} 
$\operatorname{ding}[66,67]^{29}$

$$
\begin{aligned}
& \iota: \operatorname{Sp}(2 h, \mathbb{R}) / \mathrm{U}(h) \rightarrow \operatorname{Sp}(2 h, \mathbb{R}), \\
& \iota: \mathcal{E} \mathrm{U}(h) \mapsto \mathcal{S} \stackrel{\text { def }}{=} \mathcal{E} \mathcal{E}^{t} \in \operatorname{Sp}(2 h, \mathbb{R}) \quad \text { with } \quad \mathcal{S}^{t}=\mathcal{S}, \quad \mathcal{S}>0
\end{aligned}
$$

where $\mathcal{E} \in \operatorname{Sp}(2 h, \mathbb{R})$ (called a vielbein [36]) is any chosen representative in $\operatorname{Sp}(2 h, \mathbb{R}$ ) of the given point in the coset $\operatorname{Sp}(2 h, \mathbb{R}) / \mathrm{U}(h) . \mathcal{S}$ is independent of the choice of $\mathcal{E}$. Then the map

$$
\mathcal{S} \equiv \iota \circ \widetilde{\mu}: \widetilde{\mathscr{M}} \rightarrow \operatorname{Sp}(2 h, \mathbb{R}), \quad \mathcal{S}: x \mapsto \mathcal{S}(x) \equiv \mathcal{E}(x) \mathcal{E}(x)^{t}
$$

is harmonic iff $\widetilde{\mu}$ is harmonic. The energy $E(\widetilde{\mu})$ of the gauge coupling, written in terms of $\mathcal{S}$, becomes the action of the $\operatorname{Sp}(2 h, \mathbb{R})$ principal chiral model ${ }^{30}$

$$
\frac{1}{8} \int_{\mathscr{M}} d^{n} x \sqrt{G} G^{i j} \operatorname{tr}\left(\left(\mathcal{S}^{-1} \partial_{i} \mathcal{S}\right)\left(\mathcal{S}^{-1} \partial_{j} \mathcal{S}\right)\right) \equiv \frac{1}{2} \int_{\mathscr{M}} d^{n} x \sqrt{G} G^{i j} \operatorname{tr}\left(\left(\mathcal{E}^{-1} \partial_{i} \mathcal{E}\right)^{\mathrm{o}}\left(\mathcal{E}^{-1} \partial_{j} \mathcal{E}\right)^{\mathrm{o}}\right)
$$

The tension field of the gauge coupling $\widetilde{\mu}$ is more conveniently written as

$$
T \equiv d *\left(\mathcal{S}^{-1} d \mathcal{S}\right)
$$

and $\widetilde{\mu}$ is harmonic iff $\mathcal{S}$ is a symmetric classical soliton of the chiral model defined on the space-time $\mathscr{M}$, that is, iff

$$
d *\left(\mathcal{S}^{-1} d \mathcal{S}\right)=0
$$

Symmetric means that the solution satisfies the two additional conditions $\mathcal{S}^{t}=\mathcal{S}$ and $\mathcal{S}>0$.

\subsubsection{Cartan gauge couplings}

We call the inverse matrix $\mathcal{S}^{A B}$ to $\mathcal{S}_{A B} \equiv\left(\mathcal{E E}^{t}\right)_{A B}$ the Cartan form of the gauge couplings. $\iota$ is a global isometry between the Siegel space $\boldsymbol{H}_{h}$ and the manifold of symmetric, positive, symplectic $2 h \times 2 h$ matrices, so $\mathcal{S}^{A B}$ and $\tau_{a b}$ contain exactly the same information. With respect to the usual gauge coupling $\tau_{a b}$, its Cartan version $\mathcal{S}^{A B}$ has the advantage of transforming linearly under rotations of the duality frame. All observables, being independent of the frame, have nicer expressions when written in terms of $\mathcal{S}^{A B}$. Writing $\tau_{a b}=X_{a b}+i Y_{a b}$, one has

$$
\mathcal{S}^{A B}=\left(\begin{array}{c|c}
Y^{-1} & -Y^{-1} X \\
\hline-X Y^{-1} & Y+X Y^{-1} X
\end{array}\right)^{A B} .
$$

\footnotetext{
${ }^{29}$ The target space $\operatorname{Sp}(2 h, \mathbb{R})$ is endowed with a $\operatorname{Sp}(2 h, \mathbb{R}) \times \operatorname{Sp}(2 h, \mathbb{R})$-invariant indefinite pseudoRiemannian metric, which induces on the image of $\iota$ a positive-definite Riemannian metric so that $\iota$ is an isometry onto its image (for a proper normalization of the metrics). See eq. (4.16) and footnote 30.

${ }^{30}$ The statement is a bit formal, since the action integral in the 1.h.s. of eq. (4.16) does not correspond to a positive-definite metric on the non-compact group $\operatorname{Sp}(2 h, \mathbb{R})$; all formulae are meant to be analytic continuations from the corresponding compact group $\operatorname{USp}(2 h)$. However the metric is positive-definite when restricted to the image of the Cartan map $\iota$, i.e. on the space of symmetric, positive-definite, real, symplectic $2 h \times 2 h$ matrices. As a matter of notation, when $y \in \mathfrak{s p}(2 h, \mathbb{R}), y^{\circ}$ stands for the odd part under the Cartan involution $\theta, y^{\circ} \equiv\left(y-y^{\theta}\right) / 2$, i.e. for the symmetric part of the $2 h \times 2 h$ matrix $y$. The r.h.s. of the identity (4.16) is manifestly non-negative.
} 


\section{Domestic geometry}

In $4 \mathrm{~d} \mathcal{N}=2$ supergravity the couplings of the vector-multiplets are described by special Kähler geometry, which is equivalent $[7,8]$ to the geometry of variations of Hodge structure (VHS) with non-zero Hodge numbers $h^{3,0}=h^{0,3}=1$ and $h^{2,1}=h^{1,2}=m$, where $m$ is the complex dimension of the special Kähler manifold $M$. VHS itself is a special case of Higgs bundle geometry [68], which we shall refer to as generalized $t t^{*}$ geometry. In all these geometries $M$ is a Kähler manifold. In the $\mathcal{N}=2$ SUSY case the geometric swampland problem may be rephrased as the question of which special Kähler geometries do arise as low-energy limits of consistent theories of quantum gravity. A necessary condition [15] is that the corresponding $t t^{*}$ geometry enjoys certain arithmetic properties summarized in the VHS structure theorem [58-61].

In this section we introduce a geometry - dubbed domestic - modelled on $t t^{*}$, which does not require the manifold $M$ to have a complex structure. Whenever $M$ is Kähler, domestic geometry automatically reduces to (generalized) $t t^{*}$ geometry.

We start with a review of $t t^{*}$ geometry from a viewpoint which makes natural its domestic generalization. The review is rather detailed, because we need results and formulae which cannot be found in the physical $t t^{*}$ literature. Before going to that, we recall some definitions.

Notation. We write $G(\mathbb{R})$ for a non-compact, connected, semi-simple, real Lie group with Lie algebra $\mathfrak{g}, K \subset G(\mathbb{R})$ for a maximal compact subgroup, and $G(\mathbb{Z}) \subset G(\mathbb{R})$ for a maximal arithmetic subgroup. $G(\mathbb{C})$ and $K(\mathbb{C})$ stand for the complexification of the Lie groups $G(\mathbb{R})$ and $K$, respectively.

\section{1 (Arithmetic) Tamed maps}

$M$ is an oriented Riemannian $m$-fold with a graded algebra $\mathcal{P}^{\bullet}$ of parallel forms.

Definition 3. $X$ a Riemannian manifold. A smooth map $\mu: M \rightarrow X$ is tamed iff

$$
D *(d \mu \wedge \Omega)=0 \text { for all } \Omega \in \mathcal{P}^{\bullet} .
$$

It suffices to require (5.1) for parallel forms $\Omega$ of degree $\leq m / 2$ since

$$
* D *(d \mu \wedge \Omega) \equiv(-1)^{m-1} D *(d \mu \wedge * \Omega) .
$$

Specializing to $\Omega=1$ we see that tamed $\Rightarrow$ harmonic, i.e. the tension field of a tamed map $\mu$ vanishes, $T[\mu]=0$. Written in components eq. (5.1) requires $D^{j} \partial_{i} \mu \in \operatorname{End}(T M) \otimes$ $\mu^{*} T X$ to satisfy

$$
\Omega_{j\left[i_{1} \cdots i_{k-1}\right.} D^{j} \partial_{\left.i_{k}\right]} \mu=0 \quad \text { for all } \Omega \in \mathcal{P}^{k}, \quad k=0,1, \cdots, m .
$$

We are interested only in tamed maps whose target space $X$ is a locally symmetric space of non-compact type, $X \equiv \Lambda \backslash G(\mathbb{R}) / K$, the prototypical example being a smooth finite cover of the Siegel variety

$$
\Lambda \backslash G(\mathbb{R}) / K \stackrel{\text { e.g. }}{=} \Lambda_{h} \backslash \operatorname{Sp}(2 h, \mathbb{R}) / \mathrm{U}(h) .
$$


In sections 6 and 7 below we address inter alia the following

Question. What does it mean for the gauge coupling $\mu$ in eq. (4.6) to be tamed?

We shall see that being tamed is a natural condition for the gauge couplings which has important consequences. Note that in the physical context, $\mathcal{P}^{\bullet}$ is an algebra of invariants for the continuous gauge symmetry, so $\mu$ is tamed iff the algebra of invariant tensors under the "gauge coupling endomorphisms" $D^{i} \partial_{j} \tau_{a b}$ contains all gauge invariants.

Arithmetic tamed maps. It is convenient to lift the map $\mu$ to a map between simplyconnected covers

$$
\widetilde{\mu}: \widetilde{M} \rightarrow G(\mathbb{R}) / K
$$

which is twisted by the monodromy representation $\rho: \pi_{1}(M) \rightarrow \Lambda$ of the fundamental group

$$
\widetilde{\mu} \circ \xi=\rho(\xi) \cdot \widetilde{\mu}, \quad \forall \xi \in(\operatorname{deck} \operatorname{group} \widetilde{M} \rightarrow M),
$$

that is, $\widetilde{\mu}$ is the lift which makes the following diagram to commute

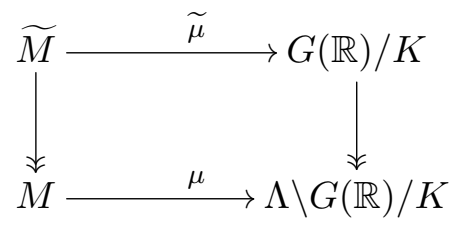

The image $\Gamma \equiv \mu_{*}\left(\pi_{1}(M)\right) \subset \Lambda$ is the monodromy group. The tamed map $\mu$ is arithmetic iff

(i) $\Lambda$, hence $\Gamma$, is a neat sub-group of a maximal arithmetic subgroup $G(\mathbb{Z})$ :

$$
\Gamma \subset \Lambda \subset G(\mathbb{Z}) \subset G(\mathbb{R})
$$

(ii) its energy is finite, $E[\mu]<\infty$.

An arithmetic tamed map is thus an instanton of the $\Lambda \backslash G(\mathbb{R}) / K \sigma$-model with some additional properties.

\subsubsection{Special cases}

For $M$ a Riemannian manifold of dimension $m \geq 3$ with generic holonomy algebra $\mathfrak{s o}(m)$, tamed is equivalent to harmonic. There are some special cases:

(a) for $M=\mathbb{R}$ : tamed $\equiv$ harmonic $\equiv$ geodesic;

(b) for $M$ strictly Kähler: tamed $\equiv$ pluri-harmonic, ${ }^{31}$ i.e. $D \bar{\partial} \mu=0$

(c) for $M$ quaternionic-Kähler (with $m \geq 8$ ): tamed $\equiv$ totally geodesic ${ }^{32}$ i.e. $D_{i} \partial_{j} \mu=0$,

\footnotetext{
${ }^{31}$ A holomorphic map between Kähler manifolds is a special instance of pluri-harmonic map.

${ }^{32}$ Let us sketch a proof. We write $a, b$ for the "flat" indices of an orthonormal frame in the tangent space $T$ at base point in $\mathcal{M}$. Let $\mathfrak{a n n}\left(\mathcal{P}^{\bullet}\right) \equiv\left\{A_{a b} \in \operatorname{End}(T): \Omega_{b\left[a_{1} \cdot a_{k-1}\right.} A_{\left.a_{k}\right] b}=0\right.$ for all $\left.\Omega \in \mathcal{P}^{\bullet}\right\}$ be the annihilator of $\mathcal{P}^{\bullet}$. From eq. (5.3) we see that $D_{a} \partial_{b} \mu \in \mathfrak{a n n} \cap \odot^{2} T$. For a strict quaternionic-Kähkler manifold of dimension $\geq 8$ one has $\mathcal{P}^{\bullet}=\mathbb{R}[\Omega]$ with $\Omega$ the canonical 4 -form. Then $\mathfrak{a n n}\left(\mathcal{P}^{\bullet}\right) \cap \odot^{2} T=0$ see proposition 1.2 of [69].
} 
(d) for $M$ locally isometric to a symmetric space $G / K$ - not of the form $\mathrm{SO}(n, 1) / \mathrm{SO}(n)$ or $\mathrm{SU}(n, 1) / \mathrm{U}(n)$ - we typically have tamed $\equiv$ totally geodesic, see appendix A for examples.

Next we consider the special case (b) in some detail. This leads to $t t^{*}$ geometry which is the model geometry which inspires all our constructions.

\subsection{Generalized $t t^{*}$ geometry}

In this subsection $M$ is a Kähler space with local holomorphic coordinates $t^{i}$.

Definition 4. A generalized $t t^{*}$ geometry (or Higgs bundle) is a tamed map from $M$ into a locally symmetric space $\Lambda \backslash G(\mathbb{R}) / K$ of non-compact type. We say that the generalized $t t^{*}$ geometry is arithmetic iff the underlying tamed map $\mu$ is arithmetic.

Let us see how this definition leads to the usual $t t^{*}$ formalism [62]. We recall that the maximal compact subgroup $K \subset G(\mathbb{R})$ is the fixed locus of a Cartan involution $\theta$ of the semi-simple Lie group $G(\mathbb{R})$. The Lie algebra $\mathfrak{g}$ of $G(\mathbb{R})$ splits into $\theta$-even and $\theta$-odd parts, $\mathfrak{g}=\mathfrak{k} \oplus \mathfrak{p}$, where $\mathfrak{k}$ is the Lie algebra of $K$ while $\mathfrak{p}$ is a $K$-module. We fix a faithful real representation $\sigma: G(\mathbb{R}) \rightarrow \mathrm{SL}(V, \mathbb{R})$ such that $V \cong V^{\vee}$, so that $G(\mathbb{R})$ is seen as a concrete group of $n \times n$ real unimodular matrices $g\left(n \equiv \operatorname{dim}_{\mathbb{R}} V\right)$. We choose conventions so that, in terms of matrices, $\theta$ is the inverse of the transpose, and we shall write $g^{t}$ for $\left(g^{-1}\right)^{\theta}$. The Maurier-Cartan form $g^{-1} d g$ is a 1 -form on the manifold $G(\mathbb{R})$ with coefficients in $\mathfrak{g} \subset \mathfrak{s l}(n)$ which may be decomposed into $\theta$-even and $\theta$-odd parts.

Let $\widetilde{\mu}: \widetilde{M} \rightarrow G(\mathbb{R}) / K$ be any smooth map twisted by the appropriate monodromy representation $\rho$, eq. (5.6). We choose a lift $f: \widetilde{M} \rightarrow G(\mathbb{R})$ of $\widetilde{\mu}$ and use it to pull-back the Maurier-Cartan form to a 1 -form on $\widetilde{M}$ with coefficients in $\mathfrak{g}$ which we decompose in $\theta$-even and $\theta$-odd parts as well as in $(p, q)$ form type

$$
f^{*}\left(g^{-1} d g\right)=A+\bar{A}+C+\bar{C}, \quad \text { where }\left\{\begin{array}{lll}
A \stackrel{\text { def }}{=} f^{*}\left(g^{-1} d g\right)_{(1,0)}^{\text {even }}, & \bar{A} & \stackrel{\text { def }}{=} f^{*}\left(g^{-1} d g\right)_{(0,1)}^{\text {even }} \\
C \stackrel{\text { def }}{=} f^{*}\left(g^{-1} d g\right)_{(1,0)}^{\text {odd }}, & \bar{C} & \stackrel{\text { def }}{=} f^{*}\left(g^{-1} d g\right)_{(0,1)}^{\text {odd }} .
\end{array}\right.
$$

We write $D \equiv \partial+A, \bar{D} \equiv \bar{\partial}+\bar{A}$ and $C \equiv C_{i} d t^{i}, \bar{C} \equiv \bar{C}_{\bar{k}} d \bar{t}^{k}$ with $C_{i}, \bar{C}_{\bar{k}} \in \mathfrak{p} \subset \mathfrak{s l}(n)$. By construction $D+\bar{D}$ is a $K$-connection on $\widetilde{M}$, while

$$
\nabla \equiv D+\bar{D}+C+\bar{C} \equiv f^{*}\left(d+g^{-1} d g\right)
$$

is a flat $G(\mathbb{R})$-connection on $\widetilde{M}$. Under a change of lift $f \rightarrow f^{\prime}=f \cdot u($ with $u: \widetilde{M} \rightarrow K$ ) both connections change by the $K$-valued gauge transformation $u$; hence the $K$-gauge invariants are independent of the chosen lift $f$ of $\tilde{\mu}$. If $\widetilde{\mu}$ is twisted by a representation $\rho$ as in eq. (5.6), the forms $A, \bar{A}, C$ and $\bar{C}$ are invariant under the action of the deck group, so they may be seen as forms on the Kähler base $M \equiv \widetilde{M} / \pi_{1}(M)$ which are canonically defined (modulo $K$-gauge transformations) by the original map $\mu: M \rightarrow \Lambda \backslash G(\mathbb{R}) / K$.

Decomposing the identity $\nabla^{2}=0$ into even/odd and form type we get the equalities

$$
\begin{aligned}
& D^{2}+C^{2}=(D C)=D \bar{D}+\bar{D} D+C \bar{C}+\bar{C} C= \\
& =(D \bar{C})+(\bar{D} C)=\bar{D}^{2}+\bar{C}^{2}=(\bar{D} \bar{C})=0
\end{aligned}
$$


which hold for all smooth maps $\widetilde{\mu}$. Now suppose that the map $\widetilde{\mu}$ is harmonic with respect to some Kähler metric $G_{k \bar{l}}$ on $\widetilde{M}$, that is, it satisfies the equation

$$
\bar{D}^{k} C_{k} \equiv G^{\bar{l} k} \bar{D}_{\bar{l}} C_{k}=0
$$

which implies the equality

$$
\bar{D}^{i} \bar{D}^{k} \operatorname{tr}\left(C_{i} C_{k}\right)=\operatorname{tr}\left[\left(\bar{D}^{i} C_{k}\right)\left(\bar{D}^{k} C_{i}\right)\right]+\operatorname{tr}\left(C_{k} \bar{D}^{i} \bar{D}^{k} C_{i}\right)
$$

while the identities (5.11) yield

$$
\begin{aligned}
& \operatorname{tr}\left[\left(\bar{D}^{i} C_{k}\right)\left(\bar{D}^{k} C_{i}\right)\right]=\operatorname{tr}\left[\left(D_{k} \bar{C}^{i}\right)\left(\bar{D}^{k} C_{i}\right)\right] \equiv\|\bar{D} C\|^{2} \\
& \operatorname{tr}\left(C_{k} \bar{D}^{i} \bar{D}^{k} C_{i}\right)=\operatorname{tr}\left(C_{k}\left[\bar{D}^{i}, \bar{D}^{k}\right] C_{i}\right)=-\operatorname{tr}\left(C_{k}\left[\bar{C}^{i}, \bar{C}^{k}\right] C_{i}\right)= \\
& =\operatorname{tr}\left(\left[C_{i}, C_{k}\right]\left[\bar{C}^{k}, \bar{C}^{i}\right]\right) \equiv\left\|\left[C_{i}, C_{k}\right]\right\|^{2} \equiv\left\|C^{2}\right\|^{2}
\end{aligned}
$$

This shows the

Lemma 1 (Sampson's Bochner-formula [70, 71]). Let $M$ be Kähler and $\mu: M \rightarrow$ $\Lambda \backslash G(\mathbb{R}) / K$ be a harmonic map. Then

$$
\bar{D}^{i} \bar{D}^{k} \operatorname{tr}\left(C_{i} C_{k}\right)=\|\bar{D} C\|^{2}+\left\|C^{2}\right\|^{2} .
$$

The r.h.s. of (5.16) is the sum of two non-negative terms: hence the integral over $M$ of the l.h.s. vanishes if and only if the two terms on the right are both identically zero; this implies (1) $\bar{D} C=0$ which (by definition) says that $\mu$ is pluri-harmonic, and (2) the algebra generated by the coefficient matrices $C_{i}$ is Abelian. In facts from eq. (5.16) we see that (2) is an automatic consequence of (1). ${ }^{33}$ The l.h.s. of (5.16) is a total derivative, so its integral over $M$ is a surface term: in particular, when $M$ is compact a harmonic map is automatically pluri-harmonic [71]. More generally:

A harmonic map $\mu: M \rightarrow \Lambda \backslash G(\mathbb{R}) / K$ is pluri-harmonic (三 tamed) if and only if

$$
\int_{\partial M} * \operatorname{tr}\left(C_{k} \bar{D}^{k} C\right) \equiv \int_{\partial M} * \operatorname{tr}\left(C_{k} D \bar{C}^{k}\right)=0,
$$

a condition which depends only on the asymptotic behaviour of $\mu$ at infinity in $M$.

The $t t^{*}$ equations. In a generalized $t t^{*}$ geometry, $\mu$ is pluri-harmonic, and hence $\bar{D} C=0$. In view of (5.11), (5.16) this implies the $t t^{*}$ PDEs [62]

$$
\begin{aligned}
D^{2}=C^{2} & =(D C)=D \bar{D}+\bar{D} D+C \bar{C}+\bar{C} C= \\
=(D \bar{C}) & =(\bar{D} C)=\bar{D}^{2}=\bar{C}^{2}=(\bar{D} \bar{C})=0 .
\end{aligned}
$$

These equations may be summarized in the following statement:

Proposition 2. For $M$ strictly Kähler, $\mu: M \rightarrow \Lambda \backslash G(\mathbb{R}) / K$ is tamed if and only if

$$
\left(\nabla^{(\zeta)}\right)^{2} \equiv\left(D+\bar{D}+\zeta^{-1} C+\zeta \bar{C}\right)^{2}=0 \text { for all } \zeta \in \mathbb{P}^{1} .
$$

\footnotetext{
${ }^{33}$ For an alternative proof that $(\mathbf{1}) \Rightarrow(\mathbf{2})$ see [65] or the appendix of [15].
} 
We write $\mathcal{R}$ for a (commutative) enveloping algebra $\mathcal{U} \mathfrak{a}$, where $\mathfrak{a} \subset \mathfrak{g l}(V, \mathbb{C})$ is a maximal commutative $\mathbb{C}$-subalgebra containing the matrices $C_{i}$. $\mathcal{R}$ is known as a chiral ring [72].

Definition 5. A $t t^{*}$ geometry is strict if it has a spectral flow i.e. we can choose $\mathcal{R}$ so that $V \cong \mathcal{R}$ as $\mathcal{R}$-modules [72]. Since $V \cong V^{\vee}$, this implies $\mathcal{R} \cong \mathcal{R}^{\vee}$ as $\mathcal{R}$-modules $\Rightarrow$ in a strict $t t^{*}$ geometry the chiral ring is a (commutative) Frobenius algebra. ${ }^{34}$ The Frobenius pairing is known as the topological metric $\eta: \mathcal{R}^{\otimes 2} \rightarrow \mathbb{C}[62,65]$.

The vacuum geometry of a $2 \mathrm{~d}(2,2)$ QFT is described by a strict arithmetic $t t^{*}$ geometry [62, 64]. The vacuum bundle ${ }^{35} \mathscr{V} \rightarrow \mathcal{M}$ over the $F$-term coupling space $\mathcal{M}$ is holomorphic with structure group $K \subset \mathrm{U}\left(\operatorname{dim}_{\mathbb{C}} \mathscr{V}\right)$, and $D+\bar{D}$ is an unitary connection on the Hermitian vector bundle $\mathscr{V}$ which coincides with the Berry connection in the quantum-mechanical sense [62]. A choice of trivialization identifies the fibers of $\mathscr{V}$ with the complexification $V_{\mathbb{C}}$ of the representation space $V$ of the real Lie group $G(\mathbb{R})$. Therefore the fibers of $\mathscr{V}$ carry a real structure ${ }^{36}$ to be identified with the physical PCT operation [62].

The chiral ring $\mathcal{R}$ is more invariantly seen as the fiber $\mathscr{R}_{t}$ of a sub-bundle $\mathscr{R} \hookrightarrow \operatorname{End}(\mathscr{V})$ consisting of commutative endomorphisms. For a generalized $t t^{*}$ the $C_{i}$ 's yield the subbundle chain

$$
T M \hookrightarrow \mathscr{R} \hookrightarrow \operatorname{End}(\mathscr{V})
$$

while for a strict $t t^{*}$ geometry

$$
T M \hookrightarrow \mathscr{V} \cong \mathscr{R} \quad \text { strict } t t^{*} \text { geometry }
$$

$t t^{*}$ metric. To simplify the notation, we write $\boldsymbol{g}$ for $f^{*} g$. Since $\boldsymbol{g} \in G(\mathbb{R})$, the connection $A+\bar{A} \equiv\left(\boldsymbol{g}^{-1} d \boldsymbol{g}\right)^{\text {even }}$ is the $K$-connection written in an unitary trivialization of the Hermitian bundle $\mathscr{V}$; more precisely, the trivialization is orthogonal because of the reality structure on $\mathscr{V}$ [62]. Since $\bar{D}^{2}=0$ the $K$-connection is also holomorphic, and it is convenient to work in a holomorphic trivialization where $\bar{A} \equiv 0$. There is a map $U: M \rightarrow K(\mathbb{C})$ such that $U \bar{D} U^{-1}=\bar{\partial}$. The transformation between the orthogonal and the holomorphic trivializations is given by the complex $K(\mathbb{C})$-gauge transformation $\boldsymbol{g} \rightarrow \boldsymbol{g} U^{-1}$. The connection $D+\bar{D}$ is both holomorphic and metric, hence is the unique Chern connection: one has

$$
A \equiv h \partial h^{-1}=\left.\left(\boldsymbol{g} U^{-1}\right)^{-1} d\left(\boldsymbol{g} U^{-1}\right)\right|_{\theta \text { even }} \quad \bar{A}=0,
$$

where $h$ is the fiber metric on $\mathscr{V}$ in the chosen holomorphic trivialization (such that the topological metric $\eta \equiv \mathbf{1}$ ). Comparing the two complex gauges

$$
h=U \bar{U}^{-1} \equiv U U^{\dagger}
$$

\footnotetext{
${ }^{34}$ See theorem 1.3 in [73].

${ }^{35}$ We recall that the vacuum bundle $\mathscr{V} \rightarrow \mathcal{M}$ is the holomorphic sub-bundle of the trivial Hilbert space bundle $\mathscr{V} \times \boldsymbol{H} \rightarrow \mathcal{M}$ whose fiber $\mathscr{V}_{t} \subset \boldsymbol{H}$ at $t \in \mathcal{M}$ is given by the subspace of zero-energy states for the Hamiltonian $H_{t}$ with $F$-term couplings $t ; \mathscr{V}$ is equipped with the sub-bundle Hermitian metric induced by the Hilbert-space Hermitian product in $\boldsymbol{H}$. The Hermitian bundle $\mathscr{V}$ and its metric are insensitive to deformations of $D$-terms couplings [62].

${ }^{36} \mathrm{~A}$ real structure on a $\mathbb{C}$-space is an anti-linear map which squares to the identity.
} 
The Hermitian metric $h$ is called the $t t^{*}$ metric on $\mathscr{V}$ [62]. It satisfies the reality condition $h \bar{h}=1[62] .{ }^{37}$

For a strict $t t^{*}$ geometry, in view of eq. (5.21), the $t t^{*}$ metric $h$ is identified with a Hermitian metric on the fibers of $\mathscr{R}$ which induces the sub-bundle metric on $T M$, i.e. a Hermitian metric on $M$. It is natural to multiply the $t t^{*}$ metric on $\mathscr{R}$ by a normalization factor so that the section $\mathbf{1} \in \mathscr{R}$ has norm 1 . In $2 \mathrm{~d}(2,2)$ QFT the normalized $t t^{*}$ metric on the coupling space $\mathcal{M}$ plays the role of the Zamolodchikov metric [62].

Hodge metric. In generalized $t t^{*}$ there is a second, better behaved metric on $M$, namely the sub-bundle metric on $T M \hookrightarrow \operatorname{End}(\mathscr{V})$ induced by the $t t^{*}$ metric on $\operatorname{End}(\mathscr{V}) \cong \mathscr{V} \otimes \mathscr{V}^{\vee}$. This metric exists independently of the spectral flow and is always Kähler [25]. Its Kähler form is

$$
i K_{i \bar{j}} d t^{i} \wedge d \bar{t}^{\bar{j}} \equiv i \operatorname{tr}(C \wedge \bar{C}) .
$$

Let $G_{i \bar{j}}$ be any Kähler metric on $M$. The $(1,1)$ tensor on $M$

$$
T_{i \bar{j}}=K_{i \bar{j}}-G_{i \bar{j}} G^{k \bar{l}} K_{k \bar{l}} .
$$

is automatically conserved

$$
\nabla^{i} T_{i \bar{j}}=G^{i \bar{h}} \nabla_{\bar{h}} K_{i \bar{j}}-G^{k \bar{l}} \nabla_{\bar{j}} K_{k \bar{l}}=G^{i \bar{h}}\left(\nabla_{\bar{h}} K_{i \bar{j}}-\nabla_{\bar{j}} K_{i \bar{h}}\right) \equiv 0 .
$$

Eq. (5.26) has a simple explanation. The map $\mu$, being pluri-harmonic, is harmonic hence a classical solution to the $\Lambda \backslash G(\mathbb{R}) / K \sigma$-model - for all choices of the "spacetime" Kähler metric $G_{i \bar{j}} . T_{i \bar{j}}$ is just the energy-momentum tensor evaluated on this on-shell field configuration and hence is conserved.

\subsubsection{HIVb brane amplitudes}

The $t t^{*}$ geometry of a $2 \mathrm{~d}(2,2)$ QFT computes important physical quantities. The basic ones are the Hori-Iqbal-Vafa half-BPS brane amplitudes (HIVb) $\Psi(\zeta)_{a}[74]$ which are sections of the bundle $\mathscr{V}^{\vee} \cong \mathscr{V}$ over $\widetilde{M},{ }^{38}$

$$
\left.\Psi(\zeta)_{a}[\phi]=\langle\phi| a \text { brane }\right\rangle, \quad \phi \in \mathscr{V} .
$$

The $\frac{1}{2}$-BPS brane amplitudes depend on a twistor parameter $\zeta \in \mathbb{P}^{1}$ which specifies the two linear combinations of the supercharges which leave them invariant [74]. The brane amplitudes are solutions to the linear PDEs

$$
\left(D+\bar{D}+\zeta^{-1} C+\zeta \bar{C}\right) \Psi(\zeta)_{a}=0
$$

and depend on a choice of $K(\mathbb{C})$-gauge (i.e. of trivialization of $\mathscr{V} \rightarrow \widetilde{M}$ ); under a change of gauge

$$
\Psi(\zeta)_{a} \rightarrow U \Psi(\zeta)_{a}, \quad U: \widetilde{M} \rightarrow K(\mathbb{C}) .
$$

The $t t^{*}$ PDEs (5.19) are the integrability conditions of the brane equation (5.28).

\footnotetext{
${ }^{37}$ In our conventions the topological metric $\eta=1$ as a consequence of our choice $g^{\theta}=\left(g^{-1}\right)^{t}$.

${ }^{38} \mathrm{Eq}$. (5.27) is written in the conventions common in the $t t^{*}$ literature [62], in particular the bracket $\langle\cdots \mid \cdots\rangle$ is linear in its first argument rather than anti-linear as in usual conventions. The index $a$ is a quantum number labelling the different fundamental branes.
} 
Fundamental solutions. A fundamental solution to eq. (5.28) is a map

$$
\Phi(\zeta): \widetilde{M} \rightarrow \sigma(G(\mathbb{C})) \subset \mathrm{SL}(n, \mathbb{C})
$$

such that the columns $\Phi(\zeta ; t, \bar{t})_{a}(a=1, \ldots, n)$ of the matrix $\Phi(\zeta) \equiv \Phi(\zeta ; t, \bar{t})$ yield a basis of linearly independent solutions of $(5.28)$. In a given $K(\mathbb{C})$-gauge, the fundamental solution is unique up to multiplication on the right by a matrix $L(\zeta) \in \sigma(G(\mathbb{C}))$ which depends only on the twistor parameter $\zeta$

$$
\Phi(\zeta ; t, \bar{t}) \rightarrow \Phi(\zeta ; t, \bar{t}) L(\zeta)
$$

In an orthogonal trivialization of $\mathscr{V}$, the matrix $L(\zeta)$ may be chosen so that $\Phi(\zeta)$ satisfies the symmetry and reality conditions

$$
\Phi(-\zeta)=\Phi(\zeta)^{\theta} \equiv\left(\Phi(\zeta)^{t}\right)^{-1}, \quad \Phi(\zeta)=\overline{\Phi(1 / \bar{\zeta})}
$$

and $\Phi\left(e^{i \theta}\right) \in G(\mathbb{R})$. In the SUSY literature it is more common to use the holomorphic gauge (cfr. eq. (5.22))

$$
\Phi(\zeta)_{\text {holo }}=U \Phi(\zeta), \quad \Phi(\zeta)_{\text {holo }}=h{\overline{\Phi(1 / \bar{\zeta})_{\text {holo }}}}_{\text {. }}
$$

which yield the usual formula for the $t t^{*}$ metric $h$ as a bilinear in the solution of the linear problem $(5.28)[62,64,65]$

$$
h=\Phi(\zeta)_{\text {holo }} \overline{\Phi(-1 / \bar{\zeta}}_{\text {holo }}^{t} .
$$

Integral structure. The HIVb fundamental brane amplitudes correspond to a special basis of solutions to (5.28), so

$$
\Psi(\zeta)_{a}=(\Phi(\zeta) L(\zeta))_{a},
$$

for some $L(\zeta)$. To get the appropriate $L(\zeta)$ note that the space of physical $\frac{1}{2}$-BPS branes has an integral structure: for $(2,2) \sigma$-models it arises because the physical branes have support on a sub-manifold of the target space, and hence represent integral elements of the relevant homology group. More generally, the integral structure arises because of Dirac quantization of the brane charge. Then the representation space $V$ of $G(\mathbb{R})$ has the form

$$
V=V_{\mathbb{Z}} \otimes_{\mathbb{Z}} \mathbb{R}
$$

with $V_{\mathbb{Z}} \subset V$ a lattice preserved by the arithmetic subgroup $G(\mathbb{Z}) \subset G(\mathbb{R})$. The brane map $\Psi(\zeta): \widetilde{M} \rightarrow \sigma(G(\mathbb{R}))$ is twisted by a monodromy representation (cfr. eq. (5.6))

$$
\xi^{*} \Psi(\zeta)=\Psi(\zeta) \cdot \rho_{\zeta}(\xi)^{-1}, \quad \forall \xi \in(\text { deck group } \widetilde{M} \rightarrow M)
$$

which should respect the arithmetic structure, so $\rho_{\zeta}(\xi) \in G(\mathbb{Z})$ and hence $\zeta$-independent. Setting $\zeta=1$ and comparing with eq. (5.6), we see that the basic brane amplitudes are given by an integral basis of solutions to (5.28) on which the monodromy action is given by multiplication on the right by $\rho(\xi)^{-1}$, where $\rho$ is the monodromy representation of the 
$t t^{*}$ geometry. Then the $t t^{*}$ metric $h$ is given by a Hermitian form in the brane amplitudes (written in a holomorphic $K(\mathbb{C})$-gauge)

$$
h=\Psi(\zeta)_{\text {holo }} I \Psi(-1 / \bar{\zeta})_{\text {holo }}^{\dagger}, \quad \text { where } I \equiv L(\zeta)^{-1}\left(L(-1 / \bar{\zeta})^{-1}\right)^{\dagger} \text {, }
$$

with $I$ the intersection form between dual bases of BPS. ${ }^{39}$

Brane amplitudes in general $t t^{*}$ geometry. The equations of the HIVb brane amplitudes, eqs. (5.28), (5.32), as well as their integral structure, continue to make sense for all generalized arithmetic $t t^{*}$ geometry, whether it has a spectral flow or not.

Given a fundamental brane amplitude $\Psi(\zeta)$ we may construct other ones by changing the representation $\sigma$ of $G(\mathbb{R})$. Usually the physical branes are given by the fundamental representation $\sigma_{\text {fund }}$; all other representations $\sigma$ are sub-representations of some $\left(\sigma_{\text {fund }}\right)^{\otimes s}$ defined by an invariant tensor $t \in \sigma \otimes\left(\sigma_{\text {fund }}^{\vee}\right)^{\otimes s}$; so the branes amplitudes associated to an arbitrary representation $\sigma$ may be interpreted as physical multi-brane amplitudes

$$
\boldsymbol{\Psi}(\zeta)_{\boldsymbol{I}}^{\boldsymbol{A}} \equiv \boldsymbol{\Psi}(\zeta)_{\boldsymbol{I}}^{{ }_{1} \cdots A_{s}}=t_{I}^{i_{1} \cdots i_{s}} \Psi(\zeta)_{i_{1}}^{A_{1}} \cdots \Psi(\zeta)_{i_{s}}^{A_{s}} \quad t_{\boldsymbol{I}}^{i_{1} \cdots i_{s}} \underset{\text { tensor. }}{G(\mathbb{R}) \text {-invariant }}
$$

\subsubsection{Graded $t t^{*}$ geometries \& VHS}

When the $2 \mathrm{~d}(2,2)$ QFT is superconformal, the $t t^{*}$ geometry has further structure induced by the superconformal $\mathrm{U}(1)_{R}$ charge. This additional structure characterizes the variations of Hodge structure inside the larger class of $t t^{*}$ geometries.

Definition 6. An arithmetic generalized $t t^{*}$ geometry is graded iff there is a grading element $Q \in i \mathfrak{g} \equiv i \mathfrak{L} \mathfrak{i}(G(\mathbb{R})$ ) (the "U(1) charge") such that

$$
[Q, A]=0, \quad[Q, C]=-C, \quad[Q, \bar{C}]=\bar{C} .
$$

A graded $t t^{*}$ geometry is a variation of Hodge structure (VHS) if, in addition,

$$
\left.e^{2 \pi i Q}\right|_{V}=(-1)^{\hat{c}}, \quad e^{i \pi Q} \in G(\mathbb{Z}), \quad \operatorname{Ad}\left(e^{\pi i Q}\right)(g)=g^{\theta} \quad \text { for } g \in G(\mathbb{R})
$$

where

$$
\hat{c} \stackrel{\text { def }}{=} 2 \max \{\text { eigenvalues of } Q \text { in } V\} \in \mathbb{N} .
$$

When (5.41), (5.42) hold, the pair $(V, Q)$ is called a Hodge representation of the Lie group $G(\mathbb{R})$ of weight $\hat{c}$. Hodge representations are classified in [61].

We identify elements of $\mathfrak{g}$ and respectively $G(\mathbb{R})$ with the matrices which represent them in the real representation space $V$; one has $g^{\theta} \equiv\left(g^{t}\right)^{-1}$ for $g \in G(\mathbb{R})$. Then eq. (5.41) implies that $\Omega \equiv e^{i \pi Q}$ is a matrix with integral entries which satisfies

$$
g^{t} \Omega g=\Omega \quad \text { for all } g \in G(\mathbb{R}),
$$

\footnotetext{
${ }^{39}$ In the most interesting case, i.e. graded $t t^{*}$ geometries, we will present the explicit form of $I$ (see eq. (5.58)) checking that $I$ is an element of $G(\mathbb{Z})$ (as physically expected), hence $\zeta$-independent.
} 
while $\Omega$ is symmetric (resp. anti-symmetric) for $\hat{c}$ even (resp. odd). Hence $V$ is an orthogonal (resp. symplectic) real representation of $G(\mathbb{R})$. The non-degenerate, integral, bilinear form

$$
\Omega(v, w) \equiv v^{t} \Omega w, \quad \text { with } \quad \Omega(v, w)=(-1)^{\hat{c}} \Omega(w, v),
$$

is called the polarization of the VHS. For a fixed $g \in G(\mathbb{R})$, we define the Weil operator

$$
C_{g}=g^{-1} e^{i \pi Q} g \in G(\mathbb{R}) .
$$

Then $\Omega\left(C_{g} v, \bar{w}\right)$ is a positive-definite Hermitian form on $V_{\mathbb{C}} \equiv V \otimes_{\mathbb{R}} \mathbb{C}$; indeed

$$
\Omega\left(C_{g} v, \bar{w}\right)=(-1)^{\hat{c}} w^{\dagger} \Omega C_{g} v=(-1)^{\hat{c}} w^{\dagger} \Omega g^{-1} \Omega g v=(-1)^{\hat{c}} w^{\dagger} g^{t} \Omega^{2} g v=w^{\dagger} g^{\dagger} g v .
$$

We write $\mathfrak{h}$ for the Lie subalgebra of $\mathfrak{g}$ which commute with $Q$, and $H \subset G(\mathbb{R})$ for the corresponding Lie subgroup. By eqs. (5.41), (5.46) the subgroup $H$ is compact, and hence contained in a maximal compact subgroup $K . G(\mathbb{R}) / H$ is then a reductive homogeneous space with a canonical projection into the symmetric space $G(\mathbb{R}) / K[75]$. Given any $H$-module $W$ we construct canonically a homogeneous bundle $\mathcal{O}(W) \rightarrow G(\mathbb{R}) / H$, with typical fiber $W$, endowed with a unique canonical connection and metric (up to overall normalization) [75]

$$
\mathcal{O}(W)=G(\mathbb{R}) \times W /\left\{(g, w) \sim\left(g h, h^{-1} \cdot w\right) \text { for } h \in H\right\} .
$$

Lemma 2. $G(\mathbb{R}) / H$ is a homogenous complex manifold, and the homogeneous vector bundle $\mathcal{O}(W) \rightarrow G(\mathbb{R}) / H$ is holomorphic for all $H$-module $W$.

Proof. Consider the grading of the complexified Lie algebra ${ }^{40}$

$$
\begin{aligned}
\mathfrak{g} \otimes \mathbb{C} & =(\mathfrak{h} \otimes \mathbb{C}) \oplus\left(\bigoplus_{r \neq 0} \mathfrak{g}^{-r, r}\right), & \text { where } \mathfrak{g}^{r,-r} \stackrel{\text { def }}{=}\{X \in \mathfrak{g} \otimes \mathbb{C}:[Q, X]=r X\}, \\
{\left[\mathfrak{h}, \mathfrak{g}^{-r, r}\right] } & \subseteq \mathfrak{g}^{-r, r}, & {\left[\mathfrak{g}^{-r, r}, \mathfrak{g}^{-s, s}\right] \subseteq \mathfrak{g}^{-r-s, r+s} . }
\end{aligned}
$$

By eq. (5.49) each summand $\mathfrak{g}^{-r, r}$ is a $H$-module, so it defines a homogeneous vector bundle $\mathcal{O}\left(\mathfrak{g}^{-r, r}\right)$. The complexified tangent bundle of the manifold $G(\mathbb{R}) / H$ is

$$
T G(\mathbb{R}) / H \otimes \mathbb{C}=\left(\bigoplus_{r>0} \mathcal{O}\left(\mathfrak{g}^{-r, r}\right)\right) \oplus\left(\bigoplus_{r<0} \mathcal{O}\left(\mathfrak{g}^{-r, r}\right)\right)
$$

We define an almost complex structure on $G(\mathbb{R}) / H$ by declaring the first summand to be the complex distribution of $(1,0)$ vectors. The almost complex structure is integrable since this complex distribution is involutive by the second equation (5.49). For the holomorphic structure of $\mathcal{O}(W)$ see e.g. [58, 75].

\footnotetext{
${ }^{40}$ In a VHS the grading is integral, $r \in \mathbb{Z}$. In this case the first equation (5.48) is an (adjoint) Hodge decomposition of $\mathfrak{g}[58-61]$.
} 
Infinitesimal period relations. The sub-bundle $\Theta \equiv \mathcal{O}\left(\mathfrak{g}^{-1,1}\right)$ of the holomorphic tangent bundle is called the Griffiths holomorphic horizontal bundle [56, 58]. Let $M$ be a complex manifold and $T M$ its holomorphic tangent bundle. We say that a map

$$
p: M \rightarrow G(\mathbb{R}) / H
$$

satisfies the Griffiths infinitesimal period relations (IPR) if $[56,58]$

$$
p_{*}(T M) \subseteq \Theta .
$$

In particular, such a map $p$ is holomorphic.

Let $V_{\mathbb{C}}=\oplus_{i} V_{q_{i}}$ be the decomposition of the $G(\mathbb{R})$-module $V_{\mathbb{C}}=V \otimes \mathbb{C}$ into eigenspaces of $Q$ of eigenvalue $q_{i}$. Since $[Q, H]=0$, each $V_{q_{i}}$ is a $H$-module and yields a homogeneous bundle $\mathcal{O}\left(V_{q_{i}}\right) \rightarrow G / H$.

Lemma 3. Let $\tilde{\mu}: \widetilde{M} \rightarrow G(\mathbb{R}) / K$ be a lift of the tamed map $\mu$ of a graded tt $t^{*}$ geometry. Then we have the factorization

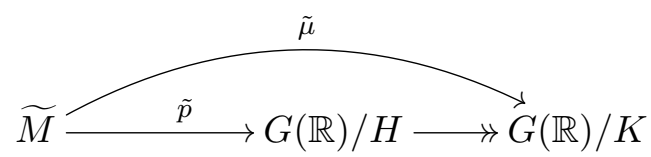

$\tilde{p}$, which is called the (Griffiths) period map of the graded $t t^{*}$ geometry, satisfies the IPR (5.52). The bundles $\mathscr{V}_{q_{i}} \equiv \tilde{p}^{*} \mathcal{O}\left(V_{q_{i}}\right) \rightarrow \widetilde{M}$ are called Hodge bundles.

Proof. In view of eq. (5.48), eqs. (5.40) are equivalent to the IPR (5.52).

Usually one identifies the graded $t t^{*}$ geometry with the period map

$$
p: M \rightarrow \Gamma \backslash G(\mathbb{R}) / H
$$

which lifts to $\widetilde{p}$ on the universal cover $\widetilde{M}$. By definition, a period map satisfies the IPR.

Applications to 2d QFT. The vacuum geometry of $(2,2) 2 \mathrm{~d}$ SCFT over the chiral conformal manifold $M$ is a graded strict $t t^{*}$ geometry. If, in addition, the $\mathrm{U}(1)_{R}$ charges of the chiral primaries [72] are integral, the SCFT vacuum geometry is a VHS of CY type, ${ }^{41}$ i.e. with Hodge number $h^{\hat{c}, 0}=h^{0, \hat{c}}=1$. The vacuum bundle $\mathscr{V} \rightarrow M$ then has an orthogonal decomposition (preserved by parallel transport with the Berry connection)

$$
\mathscr{V}=\bigoplus_{q=-\hat{c} / 2}^{\hat{c} / 2} \mathscr{V}_{q}, \quad \text { where }\left.\quad Q\right|_{\mathscr{V}_{q}}=q \in \mathbb{N}-\frac{\hat{c}}{2} .
$$

In this set-up $\hat{c}$ is one-third the Virasoro central charge [62]. The spectral-flow isomorphism is graded by the $\mathrm{U}(1)_{R^{-}}$charge,

$$
\mathscr{R}_{q} \cong \mathscr{V}_{q-\hat{c} / 2}, \quad \text { with } \quad \mathscr{R}=\bigoplus_{q=0}^{\hat{c}} \mathscr{R}_{q},
$$

and implies the "local Torelli" property $T M \cong \mathscr{V}_{1-\hat{c} / 2}$.

\footnotetext{
${ }^{41}$ For a VHS being of CY type is equivalent to being strict as a $t t^{*}$ geometry, i.e. that there is a spectral flow isomorphism.
} 


\subsubsection{Explicit graded $\mathrm{HIVb}$ brane amplitudes}

Comparing with eq. (5.28) we get an explicit formula for the twistorial multi-brane amplitudes of a graded $t t^{*}$ geometry: in the orthogonal trivialization they are $\mathbb{Z}$-linear combinations of the columns of the matrix

$$
\boldsymbol{\Psi}(\zeta)_{\boldsymbol{I}}^{\boldsymbol{A}}=\left(\zeta^{-Q} \boldsymbol{g}^{-1}\right)_{\boldsymbol{I}}^{\boldsymbol{A}} \quad \begin{aligned}
& \text { monodromy group } \\
& \text { acts on the right }
\end{aligned}
$$

with $\boldsymbol{g}_{\boldsymbol{A}}^{\boldsymbol{I}}$ the matrix elements of $\boldsymbol{g} \in G(\mathbb{R})$ in the Hodge representation $V$. Comparing with eqs. (5.30)-(5.38), we see that $L(\zeta)=\zeta^{-Q}$ and

$$
I \equiv L(\zeta)^{-1}\left(L(-1 / \bar{\zeta})^{-1}\right)^{\dagger}=e^{-\pi i Q} \stackrel{\underline{\underline{V H S}}}{=}(-1)^{\hat{c}} \Omega \in G(\mathbb{Z}),
$$

where the last equality holds in the VHS case. We see that $I$ is the natural intersection form.

\subsubsection{Physical quantities from brane amplitudes}

We can use the brane amplitudes to compute several physical quantities, that is, $K$-gauge invariant expressions which are independent of the choice of $\zeta \in \mathbb{P}^{1}$. These physical quantities are well-defined for all generalized $t t^{*}$ geometries, graded or non-graded, strict or not. Examples are

- Kähler form of Hodge metric:

- Cartan gauge coupling:

- Hodge bilinears:

$$
\begin{aligned}
i K_{i \bar{j}} d t^{i} \wedge d \overline{t^{j}} & =i \operatorname{tr}(C \wedge \bar{C}) \\
\mathcal{S}^{A B} & =\left(\Psi(\zeta)^{t} \Psi(\zeta)\right)^{A B} \\
\mathcal{S}^{A B} & =\left(\boldsymbol{\Psi}(\zeta)^{t} \Psi(\zeta)\right)^{A B} .
\end{aligned}
$$

We think of the Hodge bilinears as "higher versions" of the gauge coupling.

\subsection{5 $t t^{*}$ entropy functions \& Mumford-Tate groups}

Suppose our tamed (covering) map

$$
\tilde{\mu}: \widetilde{\mathscr{M}} \rightarrow \operatorname{Sp}(2 h, \mathbb{Z}) \backslash \mathrm{Sp}(2 h, \mathbb{R}) / \mathrm{U}(h)
$$

is actually the gauge coupling of a $4 \mathrm{~d}$ effective gravity theory. The classical ${ }^{42}$ entropy function (in Sen's sense [76-78]) of an extremal black-hole with electro-magnetic charge $q \in V_{\mathbb{Z}} \cong \mathbb{Z}^{2 h}$, (well-defined on the universal cover $\widetilde{\mathscr{M}}$ ) is

$$
E_{q}=\pi q_{A} \mathcal{S}^{A B} q_{B} \equiv \pi\|q\|_{h}^{2},
$$

that is, (up to a factor $\pi$ ) the Hodge norm-squared $\|q\|_{h}^{2}$ of the charge vector.

For a general $G$-representation $\sigma$, contained in $\otimes^{s} V$, we define the "generalized entropy functions" of the generalized $t t^{*}$ geometry to be

$$
S_{q}: \widetilde{\mathscr{M}} \rightarrow \mathbb{R}_{+}, \quad x \mapsto \boldsymbol{q}_{\boldsymbol{A}} \mathcal{S}(x)^{\boldsymbol{A} B} \boldsymbol{q}_{\boldsymbol{B}}, \quad \boldsymbol{q} \in \otimes^{s} V_{\mathbb{Z}},
$$

$S_{q}$ reduces to $E_{q} / \pi$ when $\sigma$ is the fundamental representation (i.e. $s=1$ ).

\footnotetext{
${ }^{42}$ By "classical" we mean the classical entropy function as computed by the truncation of the effective Lagrangian to two-derivatives; the classical entropy becomes exact asymptotically for large charged $|q| \rightarrow \infty$.
} 
Proposition 3. In (generalized) $t t^{*}$ geometry the generalized entropy functions $S_{\boldsymbol{q}}(t)$ are sub-pluriharmonic (in particular sub-harmonic) for all $\boldsymbol{q} \in \otimes^{s} V_{\mathbb{Z}}$, i.e. the matrix $\partial_{t^{i}} \partial_{\bar{t}_{j}} S_{\boldsymbol{q}}$ is semi-definite positive.

Proof. Set $L=\Psi(-1) \boldsymbol{q}$, so that $D L=C L, \bar{D} L=\bar{C} L, L^{*}=L$, and $S_{\boldsymbol{q}}=L^{t} L \equiv L^{\dagger} L$. Then

$$
\bar{\partial} S_{\boldsymbol{q}}=2 L^{t} \bar{D} L=2 L^{t} \bar{C} L
$$

and

$$
\begin{aligned}
\partial \bar{\partial} S_{q} & =2(D L)^{t} \bar{D} L+2 L^{t} D(\bar{C} L)=2(D L)^{t} \bar{D} L-2 L^{t} \bar{C} C L= \\
& =2 L^{\dagger}\left(C_{i} C_{j}^{\dagger}+C_{j}^{\dagger} C_{i}\right) L d t^{i} \wedge d \bar{t}^{j} .
\end{aligned}
$$

The actual value of the classical entropy for an extremal black hole of charge $q$ is given by the value $\pi S_{q}$ at a critical point $\partial S_{q}=0$ (if it exists!!). As an example, we consider the special case of a VHS of CY type with $\hat{c}=3$, which describes the gauge coupling $\widetilde{\mu}$ of an effective $4 \mathrm{~d}$ theory with $\mathcal{N}=2[7,8]$. A critical point $t_{0} \in \widetilde{\mathscr{M}}$ of $S_{q}(t)$ corresponds to a $L \equiv \Psi\left(-1 ; t_{0}\right) q$ which is an eigenvector of $Q^{2}$ with eigenvalue $9 / 4$. This observation is called the attractor mechanism [80-85]. Proposition 3 implies that the critical point is actually a minimum for the entropy function. The attractor mechanism illustrates the point that being sub-harmonic is a very natural property for a physical entropy function, being strictly related to the convexity of thermodynamical potentials.

The Mumford-Tate group. If the multi-charge $\boldsymbol{q}$ is $\Gamma$-invariant, the generalized entropy function is well-defined on $\mathscr{M}$ not just on $\widetilde{\mathscr{M}}$. Suppose that we have an arithmetic graded $t t^{*}$ geometry (say, a VHS) whose base $\mathscr{M}$ is quasi-projective, i.e. $\mathscr{M}=\overline{\mathscr{M}} \backslash D_{\infty}$ for $\overline{\mathscr{M}}$ a smooth (compact) projective variety and $D_{\infty}$ a simple normal crossing divisor; then $\mathscr{M}$ is Liouvillic for the sub-pluriharmonic functions. Under the above assumptions, one checks (by a careful asymptotic analysis [79]) that the generalized entropy of a $\Gamma$-invariant charge $\boldsymbol{q}$ is bounded along the divisors at infinity in the projective closure $\overline{\mathscr{M}}$ of $\mathscr{M}$. Then, being sub-pluriharmonic, $S_{\boldsymbol{q}}(t)$ must be a constant in $\mathscr{M}$. We conclude that for a $\Gamma$-invariant multi-charge $\boldsymbol{q} \in \otimes^{s} V_{\mathbb{Z}}$ the multi-brane amplitude $\boldsymbol{\Psi}(\zeta) \cdot \boldsymbol{q}$ is $H$-gauge equivalent to a constant; this can be expressed as

Proposition 4. Let $\mathrm{Hg}$ be the ring of all $\Gamma$-invariant multi-charges in $\oplus_{s}\left(\otimes^{s} V_{\mathbb{Z}}\right)$ and let $M(\mathbb{R}) \subset G(\mathbb{R})$ be the subgroup which fixes all elements $\boldsymbol{q} \in \mathrm{Hg}$ and $H_{M} \equiv H \cap M$ a maximal compact subgroup. Then the map $p$ in eq. (5.54) factorizes as

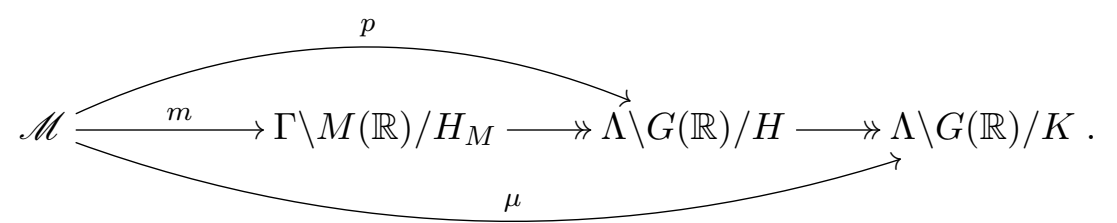

Eq. (5.67) is almost the structure theorem of VHS [58-61], except that the theorem yields more details on the group $M(\mathbb{R})$; we shall discuss these results in the more general domestic context below. In the VHS literature the elements of the $\mathbb{Q}$-algebra $\mathrm{Hg} \otimes \mathbb{Q}$ are called Hodge tensors and the $\mathbb{Q}$-algebraic group $M(\mathbb{Q})$ is called the Mumford-Tate group [58-61]. 


\subsection{Domestic geometry}

(Arithmetic) domestic geometry is defined by the very same definition 4 of $t t^{*}$ geometry, except that now we forget that the source Riemannian space $M$ was assumed to be Kähler. An arithmetic domestic geometry on the Riemannian manifold $M$ is specified by a tamed map $M \rightarrow \Lambda \backslash G(\mathbb{R}) / K$ of finite energy. The geometric structures implied by domestic geometry depend crucially on the algebra $\mathcal{P}^{\bullet}$ of parallel forms on $M$ (which we assume to be irreducible with no loss). In particular, a domestic geometry on $M$ is a generalized $t t^{*}$ geometry if and only if $\mathcal{P}^{\bullet}$ contains a subalgebra $\mathbb{R}[\omega] / \omega^{m+1}$ with $\omega$ a parallel 2 -form.

Domestic geometry is more general than $t t^{*}$ geometry as the following example shows.

\subsubsection{Example: 2d SCFT}

The (universal covering of the) conformal manifold $\widetilde{\mathcal{M}}$ of a $2 \mathrm{~d}(2,2)$ SCFT splits in a product of spaces associated to the two non-conjugate chiral rings

$$
\widetilde{\mathcal{M}}=\widetilde{\mathcal{M}}_{\text {chiral }} \times \widetilde{\mathcal{M}}_{\text {twisted chiral }},
$$

and the Berry geometry on each irreducible factor space is a domestic geometry of the $\mathfrak{u}(1)$ graded $t t^{*}$ kind (section 5.2.2).

The Berry geometry on the moduli of a $2 \mathrm{~d}(4,4)$ SCFT is still a domestic geometry, but not a $t t^{*}$ geometry. ${ }^{43}$ Indeed the domestic geometry is $\mathfrak{s p}(1) \oplus \mathfrak{s p}(1)$ graded rather than $\mathfrak{u}(1)$-graded. The moduli space $M$ is quaternionic-Kähler, and hence the underlying tamed map $\mu$ is totally geodesic (cfr. section 5.1.1): in (real) local coordinates $x^{i}$

$$
\nabla_{i} C_{j}=0, \quad \text { where } C_{j} d x^{j}=f^{*}\left(g^{-1} d g\right)^{\text {odd }} .
$$

Eq. (5.69) implies that $\widetilde{M}$ is a non-compact symmetric space with holonomy algebra of the form $\mathfrak{s p}(1) \oplus \mathfrak{s p}(1) \oplus \mathfrak{j} \subset \mathfrak{s o}(4 k)$, and hence

$$
\widetilde{M}=\mathrm{SO}(4, k) /[\mathrm{SO}(4) \times \mathrm{SO}(k)] .
$$

For a (much longer) proof not using domestic geometry, see [86].

Grading. As the 2d example illustrates, in physical applications the domestic geometry is graded by the effective R-symmetry Lie algebra $\sigma(\mathfrak{r})$, cfr. eq. (3.5), that is, we have a decomposition

$$
\mathfrak{g} \otimes \mathbb{C}=\bigoplus_{\alpha \in \operatorname{Irr}} \mathfrak{g}_{\alpha}, \quad \mathfrak{g}_{\text {adj }}=\sigma(\mathfrak{r}), \quad \mathfrak{g}_{\text {triv }}=\mathfrak{j}
$$

where the sum is over the irreducible representations of $\sigma(\mathfrak{r})$. Eq. (5.71) generalizes the adjoint Hodge decomposition (5.48) of VHS theory to a possibly non-Abelian $\sigma(\mathfrak{r})$.

\footnotetext{
${ }^{43}$ Of course, a $(4,4)$ SCFT is in particular a $(2,2)$ SCFT; however the space $M$ of marginal deformations which preserve $(4,4)$ SUSY is a non-complex submanifold of the complex manifold $\mathcal{M}$ of marginal deformations which preserve only $(2,2)$ SUSY.
} 


\subsubsection{Domestic brane amplitudes}

We may repeat much of the $t t^{*}$ story in this more general setting. However now, in general, we cannot distinguish differential forms by type, so we decompose $f^{*}\left(g^{-1} d g\right)$ in just two pieces

$$
A=\left(f^{*} g^{-1} d g\right)^{\text {even }}, \quad \Phi=\left(f^{*} g^{-1} d g\right)^{\text {odd }},
$$

and we do not have a twistorial $\mathbb{P}^{1}$-family of flat connections but only two of them

$$
\nabla^{( \pm)}=d+A \pm \Phi
$$

Consequently, we have only two "HIVb brane amplitudes" $\Psi_{ \pm}$, which satisfy the equations

$$
\nabla^{( \pm)} \Psi_{ \pm}=0
$$

As a consequence of Dirac quantization of charge, in our applications the group $G(\mathbb{R})$ preserves some bilinear pairing $V_{\mathbb{Z}} \otimes V_{\mathbb{Z}} \rightarrow \mathbb{Z}$ given by an integral matrix $\Omega \in G(\mathbb{Z})$ (symmetric or antisymmetric) with $\Omega \Omega^{t}=1$. In this case

$$
\Psi_{-}=\Psi_{+}^{\theta} \Omega, \quad \Psi_{ \pm}^{*}=\Psi_{ \pm} .
$$

Again we may consider higher domestic brane amplitudes $\boldsymbol{\Psi}_{ \pm}$associated to higher representations of the Lie group $G(\mathbb{R})$ which may be written as multi-linear products of basic brane amplitudes.

The physical quantities of $t t^{*}$ are still well-defined (we assume (5.75)):

- the Riemannian metric:

$$
\begin{aligned}
d s^{2} & =K_{i j} d x^{i} d x^{j} \equiv \operatorname{tr}\left(\Phi_{i} \Phi_{j}\right) d x^{i} d x^{j} \\
\mathcal{S}^{A B} & =\left(\boldsymbol{\Psi}_{ \pm}^{t} \boldsymbol{\Psi}_{ \pm}\right) \\
S_{\boldsymbol{q}} & =\boldsymbol{q}_{\boldsymbol{A}} \mathcal{S}^{\boldsymbol{A B}} \boldsymbol{q}_{\boldsymbol{B}}
\end{aligned}
$$

- Hodge bilinears:

- generalized entropy functions:

and the symmetric tensor

$$
T_{i j}=K_{i j}-\frac{1}{2} G_{i j} G^{k l} K_{k l}
$$

is still conserved $\left(G_{i j}\right.$ is the metric on $\left.M\right)$.

\subsection{Entropy functions in domestic geometry}

When the tamed map $\widetilde{\mu}: \widetilde{M} \rightarrow \mathrm{Sp}(2 h, \mathbb{R}) / \mathrm{U}(h)$ which defines the domestic geometry is the gauge coupling of some effective theory of gravity, Sen's classical entropy function for an extremal black hole of charge $q$ (if it exists!) is given by $\pi S_{q}$, where $S_{q}$ is the domestic entropy function for the fundamental representation. The same argument as in section 5.2.4 shows that the generalized entropy functions are sub-tamed, in particular,

- sub-harmonic for $M$ of generic holonomy: $\Delta S_{q} \geq 0$

- sub-pluriharmonic for $M$ Kähler: $\partial_{i} \bar{\partial}_{\bar{j}} S_{\boldsymbol{q}} \geq 0$

- convex for $M$ quaternionic-Kähler and most symmetric spaces: $\nabla_{i} \partial_{j} S_{\boldsymbol{q}} \geq 0$. 
Structure of $\mu$. If $M$ is Liouvillic for the sub-tamed functions and the generalized entropy function $S_{\boldsymbol{q}}$ of a $\Gamma$-invariant multi-charge $\boldsymbol{q}$ is bounded (a property natural for $\mu$ of finite energy), one concludes that the structure theorem (5.67) holds in the domestic case as well:

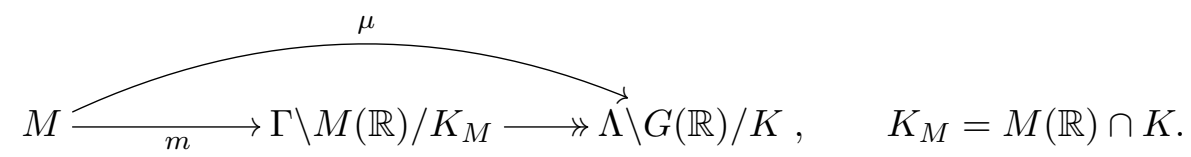

We shall show in section 7.3.3 below that this conclusion is valid in the situations of physical interest using a different and more direct approach. Here we give a rough sketch of an argument along the lines of section 5.2.5. We have already seen in section 3.1.2 that all OV manifolds $M$ are Liouvillic for the sub-harmonic functions hence a fortiori for the sub-tamed ones. It remains to show that $S_{\boldsymbol{q}}$ is bounded for $\boldsymbol{q} \Gamma$-invariant. We have to check the behaviour of $S_{\boldsymbol{q}}$ at infinity in $\widetilde{M}$. Under our assumptions in section 2.4.2, a point at infinity $x_{\infty}$ is fixed by some infinite subgroup $P \subset \Gamma \subset \operatorname{Sp}(2 h, \mathbb{Z})$ and hence is mapped by a continuous extension of $\widetilde{\mu}$ in a point at infinity $y_{\infty} \in \overline{\mathrm{Sp}(2 h, \mathbb{R}) / \mathrm{U}(h)}$ in the compactification of $\operatorname{Sp}(2 h, \mathbb{R}) / \mathrm{U}(h)$ [45] which is also fixed by $P$, i.e. such that $P y_{\infty}=y_{\infty}$. Since the entropy function

$$
S_{\boldsymbol{q}}=\widetilde{\mu}^{*}\|\boldsymbol{q}\|_{h}^{2}
$$

is the pull-back of the Hodge norm-square of $\boldsymbol{q}$ computed on $\operatorname{Sp}(2 h, \mathbb{R}) / \mathrm{U}(h)$, it suffices to check that, whenever $\boldsymbol{q}$ is fixed by $P,\|\boldsymbol{q}\|_{h}^{2}$ is bounded in a neighborhood of the point at infinity $y_{\infty}$ fixed by $P$. The last statement is a purely group theoretical fact.

The property of being sub-tamed is rather natural for a physical entropy function, as illustrated by the attractor mechanism in the $\mathcal{N}=2$ case.

\section{Domestic geometry and supergravity}

\subsection{Supergravity in $4 d$}

Consider a $4 \mathrm{~d}$ supergravity with any number $\mathcal{N}$ of light gravitini, matter content, and couplings. Its scalars' universal covering manifold ${ }^{44}$

$$
\widetilde{\mathscr{M}}=\widetilde{M}_{(1)} \times \widetilde{M}_{(2)} \times \cdots \times \widetilde{M}_{(s)}
$$

is a product of non-compact spaces in one-to-one correspondence with the types of supermultiplets present in the model. For instance, in $4 \mathrm{~d} \mathcal{N}=2$ SUGRA the scalars' manifold is the space of hypermultiplet scalars times the space of vector-multiplet scalars. The map $\widetilde{\mu}$, which describes the coupling of the scalars to the vectors, splits into a set of maps $\left\{\widetilde{\mu}_{(i)}\right\}$ which describe the coupling of vectors to the scalars in supermultiplets of the $i$-th type.

The geometry of each factor space $\widetilde{M}_{(i)}$ depends on the corresponding supermultiplet. Prima facie these geometries look quite different one from the other: in some cases $\widetilde{M}_{(i)}$ is Kähler (possibly with additional structures), in other situations $\widetilde{M}_{(i)}$ does not even admit

\footnotetext{
${ }^{44}$ As always, we work modulo finite groups and finite covers.
} 
a natural complex structure. A general feature is that each space $\widetilde{M}_{(i)}$ carries a nontrivial algebra $\mathcal{P}_{(i)}^{\bullet}$ of parallel forms determined by the representation of R-symmetry on the scalars as described in section 3.1.1.

Domestic geometry unifies all these seemingly different geometries in a single one. The traditional supergravity theory, as well as the geometric swampland conjectures, may be summarized in the following statement:

Fact 1. All 4d SUGRA models are defined by a domestic geometry:

1. The $\Gamma$-twisted (covering) gauge coupling map $\tilde{\mu}: \widetilde{\mathscr{M}} \rightarrow \mathrm{Sp}(2 h, \mathbb{R}) / \mathrm{U}(h)$ is always tamed.

2. More precisely, for each factor manifold $\widetilde{M}_{(i)}$ in eq. (6.1) there is a $\Gamma_{(i)}$-twisted, $\mathcal{P}_{(i)}^{\bullet}{ }^{-}$ tamed map

$$
\widetilde{\mu}_{(i)}: \widetilde{M}_{(i)} \rightarrow G_{(i)} / K_{(i)}, \quad \Gamma_{(i)} \subset G_{(i)}
$$

with target a symmetric space $G_{(i)} / K_{(i)}$ and $\tilde{\mu}$ factorizes as in the commutative diagram

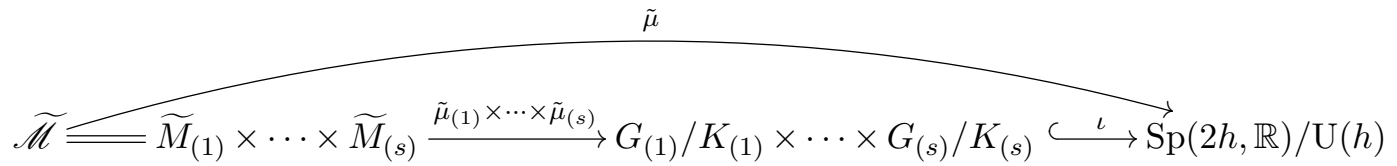

where $\iota$ is the totally geodesic embedding induced by a subgroup embedding

$$
G_{(1)} \times \cdots \times G_{(s)} \stackrel{\iota}{\hookrightarrow} \mathrm{Sp}(2 h, \mathbb{R})
$$

and $\Gamma \sim \prod_{i} \Gamma_{(i)}$ modulo finite groups;

3. Most couplings in the Lagrangian $\mathscr{L}$ of an (ungauged) $4 d S U G R A$ are given by universal expressions in terms of brane amplitudes of the domestic geometries $\widetilde{\mu}_{(i)}$. For $\mathcal{N} \geq 3$ and the vector sector of $\mathcal{N}=2$ ALL couplings are so expressed;

4. The domestic geometry rigidity theorems/structure theorems reproduce and extend the usual SUSY non-renormalization theorems;

5. If the domestic geometry defined by a map $\widetilde{\mu}_{(i)}$ in (6.2) is non-arithmetic the SUGRA falls in the swampland.

The statement holds, with the appropriate modifications, in other spacetime dimensions.

As we have seen in section 5.1.1, there are several special cases of domestic geometry depending on the particular algebra $\mathcal{P}^{\bullet}$; when the domestic geometry is a VHS, we may talk of its Hodge numbers and its Mumford-Tate (MT) group. In table 1 we list the domestic geometries which arise in 4d SUGRA with the special properties of each one.

Remark 5. For the benefit of the reader we recall on which grounds each statement in fact 1 rests. Items 1. and 3. are a rephrasing of well-known facts about 4d SUGRA (see e.g. the textbook [36]); they are perfectly rigorous in the sense of classical Lagrangian field theory. 


\begin{tabular}{|c|c|c|c|c|c|}
\hline $\mathcal{N} \&$ supermultiplet & $\begin{array}{l}\text { name } \\
\text { space }\end{array}$ & $\mathbb{C} ?$ & $\begin{array}{l}\text { - kind of domestic geometry } \\
\text { - algebra } \mathcal{P}^{\bullet} \text { if special }\end{array}$ & $\begin{array}{l}\text { - for } \operatorname{VHS} h^{p, q} \neq 0 \\
\text { - MT group if special }\end{array}$ & notes \\
\hline $\mathcal{N}=1$ chiral & & $\checkmark$ & graded $\hat{c}=1$ non-strict $t t^{*}$ & $\left\{h^{1,0}\right\}$ & \\
\hline $\mathcal{N}=2$ vector & SKG & $\checkmark$ & graded $\hat{c}=3$ strict $t t^{*}$ & $\left\{h^{3,0}=1, h^{2,1}\right\}$ & \\
\hline $\mathcal{N}=2$ hyper & QK & NO & $\begin{array}{l}\text { domestic tamed by } \\
\mathcal{P}^{\bullet}=\mathbb{C}[1, \Omega](\Omega \text { canonical 4-form })\end{array}$ & & \\
\hline $\mathcal{N}=3$ vector & & $\checkmark$ & $\begin{array}{l}\text { graded } \hat{c}=3 \text { non-strict } t t^{*} \text { tamed } \\
\text { by } \mathcal{P}^{\bullet}=\left[\wedge^{*}\left(\mathbb{C}^{3} \otimes \mathbb{C}^{k}\right)\right]^{\mathfrak{u}(3) \oplus \mathfrak{s u}(k)}\end{array}$ & $\begin{array}{l}\left\{h^{3,0}=3, h^{2,1}=k\right\} \\
\Gamma^{\mathbb{Q}}(\mathbb{R})=\mathrm{SU}(3, k)\end{array}$ & $\mathrm{LU}, \mathrm{SH}$ \\
\hline $\mathcal{N}=4$ gravity & $\boldsymbol{H}_{1}$ & $\checkmark$ & graded $\hat{c}=1$ strict $t t^{*}$ & $\left\{h^{1,0}=1\right\}$ & $\mathrm{LU}, \mathrm{SH}$ \\
\hline $\mathcal{N}=4$ vector & & NO & $\begin{array}{l}\text { domestic tamed by } \\
\mathcal{P}^{\bullet}=\left[\wedge^{*}\left(\mathbb{R}^{6} \otimes \mathbb{R}^{k}\right)^{\mathfrak{s o}(6) \oplus \mathfrak{s o}(k)}\right.\end{array}$ & & $\mathrm{LU}$ \\
\hline $\mathcal{N}=5$ gravity & & $\checkmark$ & $\begin{array}{l}\text { graded } \hat{c}=1 \text { strict } t t^{*} \text { tamed } \\
\text { by } \mathcal{P}^{\bullet}=\left[\wedge^{*}\left(\wedge^{4} \mathbb{C}^{5}\right)^{\vee}\right]^{\mathfrak{u}(5)}\end{array}$ & $\begin{array}{l}\left\{h^{1,0}=10\right\} \\
\Gamma^{\mathbb{Q}}(\mathbb{R})=\operatorname{SU}(5,1)\end{array}$ & $\mathrm{LU}, \mathrm{SH}$ \\
\hline $\mathcal{N}=6$ gravity & $\widetilde{\mathrm{SKG}}$ & $\checkmark$ & $\begin{array}{l}\text { graded } \hat{c}=3 \text { strict } t t^{*} \text { tamed by } \\
\mathcal{P}^{\bullet}=\left[\wedge^{*}\left(\wedge^{4} \mathbb{C}^{6}\right)\right]^{\mathfrak{u}(6)}\end{array}$ & $\begin{array}{l}\left\{h^{3,0}=15, h^{2,1}=1\right\} \\
\Gamma^{\mathbb{Q}}(\mathbb{R})=\operatorname{SO}(6, \mathbb{H})\end{array}$ & LU, SH \\
\hline $\mathcal{N}=8$ gravity & & NO & $\begin{array}{l}\text { domestic tamed by } \\
\mathcal{P}^{\bullet}=\left[\wedge^{*}\left(\wedge_{+}^{4} \mathbb{C}^{8}\right)\right]^{\mathfrak{s u}(8)}\end{array}$ & & $\mathrm{LU}$ \\
\hline
\end{tabular}

Table 1. 4d SUGRAs as domestic geometries. In second column SKG stands for special Kähler geometry, QK for quaternionic-Kähler, $\widetilde{\mathrm{SKG}}$ for twisted special Kähler (with $e^{i \pi Q} \rightarrow-e^{i \pi Q}$ ), and $\boldsymbol{H}_{1}$ for the upper half-plane. In third column $\checkmark$ means that the manifold has a natural complex structure. Forth column specifies the class of tamed geometries and its taming algebra $\mathcal{P}^{\bullet}$. In fifth column we specify the data for domestic geometries which are in fact VHS. In the last column LU means that the geometry is locally unique (so $\widetilde{M}_{(i)}$ is a symmetric space) and SH that, under the assumption that the domestic geometry is arithmetic $M_{(i)} \equiv \Gamma_{(i)} \backslash \tilde{M}_{(i)}$ is a Shimura variety.

Item 2 follows from 1. and 3. together with the original Ooguri-Vafa swampland conjectures (which we take as "facts" for the purpose of the present paper). In other words: it follows (rigorously) from the above standard facts in supergravity together with the assumption that the scalars' manifold is of the OV class. More in detail: for $\mathcal{N} \geq 3$ SUGRA item 2 . is shown in section 4.9 of [36] and for $\mathcal{N}=2$ SUGRA in refs. [15, 28]. In $\mathcal{N}=1$ SUGRA the gauge coupling $\tau_{i j}$ is a holomorphic map between the scalars' Kähler manifold and the Siegel variety $\mathrm{Sp}(2 h, \mathbb{Z}) \backslash \mathrm{Sp}(2 h, \mathbb{R}) / \mathrm{U}(h)$. Then, by definition, $\tau_{i j}$ is the formal period map of a weight-1 VHS; since item 2 is just the structure theorem for period maps in VHS, we need only to check that the formal period map $\tau_{i j}$ satisfies the conditions of the structure theorem. The VHS structure theorem is a consequence of the theorem of the fixed part in VHS: hence it is enough to verify that this last theorem holds for $\tau_{i j}$; going through the details of Schmid's proof [79] of the fixed part theorem, we see that its statement holds if the scalars' Kähler space is Liouvillic for the sub-pluriharmonic functions. This property holds for Kählerian OV manifolds, and item 2 follows for $\mathcal{N}=1$. Item 4 . is a consequence of item 3: non-renormalization of a Lagrangian coupling means that it cannot be deformed continuously (in a consistent way); traditionally the SUSY non-renormalization properties are attributed to "the power of holomorphy" [21]. However holomorphy is just the cheaper way of implementing rigidity of the HIVb which determines the coupling. It is obvious that rigidity of the branes suffices to rule out corrections to the associated couplings, while 
rigidity applies to a larger class of situations than holomorphy. Conversely, if the coupling is undeformable, the associated brane is rigid. Item 5. summarizes the previous discussion of arithmetic domestic geometry and its relation to Dirac quantization of charge.

\subsection{Swampland vs. brane amplitudes}

The above fact allows for a more physical interpretation of the Ooguri-Vafa geometric swampland conditions. For $\mathcal{N} \geq 2$ the couplings are expressed in terms of brane amplitudes, and the swampland conjectures just say that these brane amplitudes have the properties we expect on physical grounds for actual extended objects.

The brane viewpoint makes the swampland story a lot less mysterious. Tautologically, a theory is consistent iff it leads to physically sound predictions for all observables. In, say, $\mathcal{N}=2$ supergravity the brane amplitudes are important physical quantities: if they don't behave in the correct way, the theory is doomed to be inconsistent. This is what (typically) happens when the swampland conjectures of [2] are not obeyed.

\subsection{Supergravity as moduli-space gravitational instantons}

From table 1 we see that in all 4d SUGRAs the gauge coupling maps

$$
\mu_{(i)}: \Gamma_{(i)} \backslash \widetilde{M}_{(i)} \rightarrow \Gamma_{(i)} \backslash G_{(i)} / K_{(i)}
$$

are harmonic, hence solutions to the $\sigma$-model with target space $\Gamma_{(i)} \backslash G_{(i)} / K_{(i)}$ and action

$$
S\left[\mu_{(i)}\right]=\frac{1}{2} \int_{\Gamma_{(i)} \backslash \widetilde{M}_{(i)}} d^{n_{(i)}} x \sqrt{\operatorname{det} g_{(i)}} g_{(i)}^{k l} h(\mu)_{a b} \partial_{k} \mu_{(i)}^{a} \partial_{l} \mu_{(i)}^{b}
$$

where $g_{(i) k l}$ is the kinetic-term metric on the $i$-th factor space $\widetilde{M}_{(i)}$.

If our SUGRA model is not in the swampland, the solutions $\mu_{(i)}$ have finite energy (action) $S\left[\mu_{(i)}\right]<\infty$. Thus a partial answer to the simpler question on page 21 is that, in the SUSY case, the tension field of the gauge couplings should vanish while their energy must be finite.

Although the above statements are fully correct, they looks a bit unsatisfactory. We are treating the couplings $g_{(i)}$ and $\mu_{(i)}$ asymmetrically - the first one as a background metric on $\widetilde{M}_{(i)}$ and the second one as a classical dynamical field - while the two couplings are on the same footing in the swampland story, in facts geometrically unified in the $3 \mathrm{~d}$ scalars' manifold $\mathscr{M}_{3}$ (cfr. the "total space" viewpoint in section 4.2). Then it is natural to treat also the moduli metrics $g_{(i)}$ as classical dynamical fields.

A naive proposal will be to replace the $i$-th $\sigma$-model action by its minimal coupling to gravity (allowing for a cosmological constant), that is, to consider the following theory living on the moduli space $M_{(i)}$

$$
S\left[g_{(i)}, \mu_{(i)}\right]=\int_{\Gamma_{i} \backslash \widetilde{M}_{i}} d^{n_{i}} \varphi \sqrt{\operatorname{det} g_{(i)}}\left(-\frac{1}{2 \kappa_{(i)}^{2}} R_{(i)}+\Lambda_{(i)}+\frac{1}{2} g_{(i)}^{k l} h(\mu)_{a b} \partial_{k} \mu_{(i)}^{a} \partial_{l} \mu_{(i)}^{b}\right) .
$$

However the $\mathscr{M}_{3}$ "total space" viewpoint of section 4.2 suggests that additional KK fields must live on $\mathscr{M}$, so the proposal (6.6) looks a bit naive, and we should not expect it to 
work in full generality. If we are lucky, $S\left[g_{(i)}, \mu_{(i)}\right]$ may at best be a consistent truncation of the moduli-space gravity theory (if it exists!).

Claim. In $4 d \mathcal{N} \geq 2 S U G R A$ all couplings $g_{(i)}, \mu_{(i)}$ are solutions to the classical equations of motion following from the action $S\left[g_{(i)}, \mu_{(i)}\right]$ for appropriate constants $\kappa_{(i)}, \Lambda_{(i)}$. If the SUGRA arises as the low-energy description of a consistent quantum theory of gravity, the solutions have finite action, i.e. are gravitational instantons on $M_{(i)} \equiv \Gamma_{(i)} \backslash \widetilde{M}_{(i)}$.

Indeed, the equation of motion for the moduli-scalars are satisfied since the $\mu_{(i)}$ are harmonic. One has only to check that the Einstein equations

$$
R_{(i) k l}-\frac{1}{2} g_{(i) k l} R_{(i)}+\kappa_{(i)}^{2} \Lambda_{(i)} g_{(i) k l}=\kappa_{(i)}^{2} T_{(i) k l}
$$

hold for some constants $\kappa_{(i)}^{2}, \Lambda_{(i)}$. Equivalently, it is enough to show that the three symmetric tensors $R_{(i) k l}-\frac{1}{2} g_{(i) k l} R_{(i)}, g_{(i) k l}$, and $T_{(i) k l}$ are not linearly independent. For $\mathcal{N} \geq 3$ all factor spaces $M_{(i)}$ are locally symmetric, hence Einstein $R_{(i) k l}=-\lambda_{(i)} g_{(i) k l}$, while the gauge coupling $\widetilde{\mu}_{(i)}: \widetilde{M}_{(i)} \rightarrow G_{(i)} / K_{(i)}$ is an isometry ${ }^{45}$ (up to overall normalization), so that the three tensors are equal up to an overall constant and claim holds. For $\mathcal{N}=2$ one has two factor spaces $\widetilde{M}_{\text {hyper }}$ and $\widetilde{M}_{\text {vector }} \cdot \widetilde{\mu}_{\text {hyper }}$ is the contant map, so $T_{\text {hyper } k l} \equiv 0$, while $\widetilde{M}_{\text {hyper }}$, being negative quaternionic-Kähler, is Einstein, so eq. (6.7) holds. The tricky case is $\widetilde{M}_{\text {vector }}$, which is a special Kähler manifold. That the Einstein equations (6.7) hold in this case was shown in [28]. Finally the last statement follows from the fact that evaluated on the appropriate classical solution the action density is proportional to the volume form [28], so that finite action is equivalent to finite volume of $M_{(i)}$, which is one of the swampland conjectures.

The claim may be regarded as a general geometric rigidity theorem, alias a general SUSY non-renormalization theorem. E.g. the $\mathcal{N}=2$ case yields the two non-renormalization theorems of $\mathcal{N}=2$ SUGRA.

$\mathcal{N}=1 S U G R A$. In the $\mathcal{N}=1$ case we have weaker non-renormalization theorems, so we cannot expect that the story is as simple as for $\mathcal{N} \geq 2$. We have a non-renormalization theorem for $F$-term couplings, so we expect the gauge coupling $\mu$ to be still a tamed map; this is of course correct, since the gauge coupling is holomorphic and a fortiori pluri-harmonic. The moduli metric, however, is not expected to be a solution of the Einstein equations following from a simple action of the form (6.6) since we don't have the corresponding non-renormalization theorem. One may speculate about more complicated "dynamical" equations for the $\mathcal{N}=1$ moduli metric $g(\phi)_{i j}$ with additional degrees of freedom propagating on the scalars' moduli space $\mathscr{M}$. Some proposal will be discussed elsewhere.

\section{Domestic geometry and the swampland}

In the supersymmetric context the geometric swampland conditions may be conveniently rephrased as the requirement that the underlying domestic geometry is arithmetic, i.e. as

\footnotetext{
${ }^{45}$ More precisely, we may choose $G_{(i)}$ as small as possible and then $\widetilde{\mu}_{(i)}$ is an isometry; if we choose $G_{(i)}$ non-minimal, $\widetilde{\mu}_{(i)}$ is just a totally geodesic embedding.
} 
the statement that quantum-consistent effective models have formal brane amplitudes with the right properties to correspond to actual physical branes. Since domestic geometry and its brane amplitudes $\Psi_{ \pm}$make sense on all Riemannian manifolds, one is naturally led to ask whether the statement is true for all quantum-consistent effective theories of gravity, supersymmetric or not.

We have no quantitative control on the quantum-consistency of non-supersymmetric effective theories, so the question is really a matter for speculation. There are, however, several reasons to believe that domestic geometry is somehow on the right track even in the non-SUSY case:

- evidence from the SUSY examples;

- the elegant and deep connection between domestic geometry and the Ooguri-Vafa geometric swampland conjectures which apply in general, not just in the SUSY context;

- domestic geometry implies physically desirable properties of the entropy functions;

- physical "naturalness" considerations, see the discussion in subsubection 7.2.3 and 7.2.4.

\subsection{Domestic geometry vs. OV manifolds}

OV manifolds are the natural arena for tamed maps. Indeed

Tamed property. $\mathscr{M}$ a $O V$ manifold and $G(\mathbb{R}) / K$ a symmetric space of non-compact type. All maps $\mu: \mathscr{M} \rightarrow \Lambda \backslash G(\mathbb{R}) / K$ of finite energy (action) may be continuously deformed into a unique tamed map $\dot{\mu}$ which is the map of minimum energy in its homotopy class. In particular, all harmonic maps $\mu: \mathscr{M} \rightarrow \Lambda \backslash G(\mathbb{R}) / K$ which have finite energy (action) are automatically tamed.

We defer the argument to the end of section 7.3.2.

We assume as our working hypothesis that, in a quantum-consistent theory of gravity, the low-energy gauge coupling $\mu: \mathscr{M} \rightarrow \operatorname{Sp}(2 h, \mathbb{Z}) \backslash \mathrm{Sp}(2 h, \mathbb{R}) / \mathrm{U}(h)$ has finite energy (action). This holds in all known (supersymmetric) examples constructed from the superstring, where this condition is strictly related to various swampland considerations [15], and looks quite reasonable in general. Under this hypothesis, the actual gauge coupling $\mu$ is a continuous deformation of a well-defined tamed map $\stackrel{\leftrightarrow}{\mu}$, so domestic geometry is at least "qualitatively correct". The "correction" $\mu-\stackrel{\rho}{\mu}$ vanishes in the SUSY case, and we shall speculate that it should be small (or even zero) in general.

We stress that, for $\mathscr{M}$ an OV manifold, the tamed map $\stackrel{\mu}{\mu}$ is uniquely determined by the action on the scalars of the continuous and discrete (bosonic) gauge symmetries. Under our working assumption, the action of the gauge symmetries on $\mathscr{M}$ is then restricted by the condition that a finite-energy tamed map $\stackrel{\mu}{\mu}$ does exist. This yields a severe condition on $\pi_{1}(\mathscr{M})$ which may be regarded as a stringent refinement of the swampland $\pi_{1}$-conjecture of [2]. 


\subsection{Tension flow and naturalness of the gauge couplings}

When the source is an OV manifold and the target is an arithmetic quotient of a symmetric space without compact factors, the conditions of being arithmetic tamed and being harmonic are equivalent for maps of finite energy by the tamed property. Then, to construct an arithmetic domestic geometry on an OV space $\mathscr{M}$, it suffices to impose that the relevant map $\mu: \mathscr{M} \rightarrow \Lambda \backslash G(\mathbb{R}) / K$ is harmonic of finite energy. This last condition has a simple interpretation which we now review.

\subsubsection{Tension flow}

Let $M, N$ be Riemannian manifolds. In order to construct a harmonic map $\phi: M \rightarrow N$ in a given homotopy class (equivalently, a covering harmonic map $\tilde{\phi}: \widetilde{M} \rightarrow \widetilde{N}$ twisted by a given monodromy representation $\rho$ of $\pi_{1}(M)$ ) one may think of starting with an arbitrary smooth map $\phi_{0}$ in that class which has finite energy $E\left(\phi_{0}\right)<\infty$ (if it exists!), and then continuously deform it to decrease its energy until we reach a minimum value. A convenient way of implementing this variational strategy, pionereed by Eells and Sampson [87], is the tension flow. One considers a family of maps $\phi_{t}: M \rightarrow N$, parametrized by $t \in \mathbb{R}$, which satisfies the differential equation ${ }^{46}$

$$
\frac{d \phi_{t}}{d t}=D * d \phi_{t} \equiv T\left(\phi_{t}\right) \equiv-\operatorname{grad} E\left(\phi_{t}\right)
$$

with initial value the finite energy map $\phi_{0}$. If the solution to the PDE (7.1) exists and its limit as $t \rightarrow+\infty$ is smooth, $\phi_{\infty}$ is a finite-energy harmonic map in the homotopy class of $\phi_{0}$. The existence problem for harmonic maps is then reduced to showing that the solution to the gradient flow (7.1) exists and its limit is regular.

For a family of $\rho$-twisted maps $\widetilde{\mu}_{t}: \widetilde{\mathscr{M}} \rightarrow \operatorname{Sp}(2 h, \mathbb{R}) / \mathrm{U}(h)$ the flow equation takes the elegant form (cfr. eq. (4.18))

$$
\mathcal{S}_{t}^{-1} \frac{d \mathcal{S}_{t}}{d t}=D^{i}\left(\mathcal{S}_{t}^{-1} \partial_{i} \mathcal{S}_{t}\right)
$$

where $\mathcal{S}_{t}=\left(\mathcal{S}_{t}\right)^{t}>0$ is the composition of $\widetilde{\mu}_{t}$ with the Cartan diffeomorphism $(4.14)^{47}$

$$
\iota: \operatorname{Sp}(2 h, \mathbb{R}) / \mathrm{U}(h) \equiv \boldsymbol{H}_{h} \stackrel{\sim}{\rightarrow} \operatorname{Sp}(2 h, \mathbb{R}) \cap P(2 h, \mathbb{R}) .
$$

The derivative $D^{i}$ in (7.2) is covariant only with respect to the Levi-Civita connection on the source space $\widetilde{\mathscr{M}}$. From the form eq. (7.2), it is obvious that along the flow one has $\mathcal{S}_{t} \in \operatorname{Sp}(2 h, \mathbb{R})$ and $\left(\mathcal{S}_{t}\right)^{t}=\mathcal{S}_{t}>0$ for all $t$.

The tension flow has many analogies with the well known Ricci flow on a manifold $M$ (for a review see [33]) which we may roughly see as the RG flow of the $2 \mathrm{~d} \sigma$-model with target $M$. The analogy is not accidental; indeed the tension flow is a special instance of Ricci flow as we are going to show. ${ }^{48}$

\footnotetext{
${ }^{46} \mathrm{grad}$ stands for the gradient in the Banach manifold of smooth maps from $M$ to $N$. Cfr. eq. (4.10).

${ }^{47} P(2 h, \mathbb{R})$ stands for the space of positive-definite $2 h \times 2 h$ real symmetric matrices.

${ }^{48} \mathrm{~A}$ different application of the Ricci flow to the swampland program has been discussed in [34].
} 


\subsubsection{Relation to Ricci flow}

Before addressing the question of existence of harmonic maps, let us explain the relation of the gradient flow (7.1) with the Ricci flow in a context where the map $\mu: \mathscr{M} \rightarrow \Lambda \backslash \boldsymbol{H}_{h}$ is the gauge coupling of a $4 \mathrm{~d}$ field theory. For simplicity we first consider an effective model with only scalars and Abelian vectors (no gravity or fermions)

$$
\mathscr{L}_{\text {eff }}=-\frac{1}{2} F_{\pi}^{2} G(\phi)_{i j} \partial^{\mu} \phi^{i} \partial_{\mu} \phi^{j}-\frac{i}{16 \pi} \tau(\phi)_{a b} F_{+}^{a} F_{+}^{b}+\frac{i}{16 \pi} \bar{\tau}(\phi)_{a b} F_{-}^{a} F_{-}^{b}
$$

which we interpret as a field theory with an explicit UV cut-off $\Lambda_{\text {eff }}$ at the energy scale where the IR description breaks down. The scalar fields $\phi^{i}$ are seen as adimensional local coordinates on $\mathscr{M}$, and $F_{\pi}$ is the overall mass scale of their kinetic terms. Except in section 7.2 .3 we set $F_{\pi}=1$.

We compactify this $4 \mathrm{~d}$ model to $3 \mathrm{~d}$ on a circle of radius $R$. Each $4 \mathrm{~d}$ vector yields two real scalars in $3 \mathrm{~d}$ : one from the internal component $A_{4}^{a}$ and one from the dual to $A_{\mu}^{a}$. The $2 h$ scalars arising from the $4 \mathrm{~d}$ vectors are periodic since they correspond to the electric and magnetic $\mathrm{U}(1)^{h}$ holonomies along the circle; we parametrize the $2 h$ holonomies as $\exp \left(2 \pi i y^{A}\right)(h=1, \ldots, 2 h)$.

The resulting $3 \mathrm{~d}$ effective theory is the $\sigma$-model with scalars' manifold the total space of the fibration $\mathscr{X} \rightarrow \mathscr{M}$ mentioned in section 4.2: the fiber $\mathscr{X}_{\phi}$ is a $h$-dimensional Abelian variety (over $\mathbb{C}$ ) with periods $\tau(\phi)_{a b}$ and fixed principal polarization (which we identify with its Kähler class). The total space $\mathscr{X}$ is equipped with the metric

$$
d s_{3 \mathrm{~d}}^{2}=R G(\phi)_{i j} d \phi^{i} d \phi^{j}+\frac{1}{R} \mathcal{S}(\phi)_{A B} d y^{A} d y^{B}+(\text { exponentially small as } R \rightarrow \infty)
$$

where $\mathcal{S}_{A B} \equiv\left(\mathcal{E E}^{t}\right)_{A B}$ is the inverse of the Cartan coupling $\mathcal{S}^{A B}$. The exponentially small corrections are due to $4 \mathrm{~d}$ massive particles, carrying electro-magnetic charges, whose worldline wrap the circle; such corrections are well studied in the context of $3 \mathrm{~d}$ compactifications of $4 \mathrm{~d} \mathcal{N}=2$ QFT [94]. We shall take $R$ large and ignore the exponential corrections.

Compactifying further down to two dimensions, we get a $2 \mathrm{~d} \sigma$-model with target space metric proportional to (7.5) whose RG flow is given (in the one-loop approximation) by the Ricci flow. The flow preserves the structure of the metric (7.5) so it decomposes into a pair of equations of the form

$$
R \frac{d}{d t} G(\phi)_{i j}=-2 R(\phi)_{i j}, \quad \mathcal{S}^{A C} \frac{d}{d t} \mathcal{S}(\phi)_{C B}=-2 R(\phi)^{A}{ }_{B}
$$

The Ricci curvature in the fiber directions is easily computed to be

$$
R_{B}^{A}=-\frac{1}{2} D^{i}\left(\mathcal{S}^{-1} \partial_{i} \mathcal{S}\right)_{B}^{A} .
$$

Comparing with eq. (7.2), we see that the Ricci flow restricted to the fibers has the same form as the original tension flow. However, in general, the two flows in (7.6) are coupled together because the covariant derivative $D^{i}$ depends on the evolving metric $G_{i j}$ on the base. When the base $\mathscr{M}$ is Ricci-flat or Einstein, the two flows are identical. 
Note that $R_{A}^{A}=0$, so that the Ricci flow preserves the volume of the Abelian fibers. In facts, the flow preserves the fiber's Kähler form (三 polarization) while changing its complex structure. To see this, we introduce the orthonormal co-frame

$$
e^{m} \equiv \mathcal{E}_{A}^{m} d y^{A} \quad \mathcal{E} \in \operatorname{Sp}(2 h, \mathbb{R})
$$

where $\mathcal{E}$ is the vielbein in (4.14). The isotropy group $\mathrm{U}(h)$ acts on the "flat" index $m$ in the representation $\boldsymbol{h} \oplus \overline{\boldsymbol{h}}$, defining on the fiber a torsion-less flat $\mathrm{U}(h)$-structure hence an integrable complex structure and a closed Kähler form which is given in "flat" indices by the constant symplectic matrix $\Omega_{m n}$. Then the fiber Kähler form is

$$
\omega_{\text {fiber }} \stackrel{\text { def }}{=} \Omega_{m n} e^{m} \wedge e^{n}=\left(\mathcal{E} \Omega \mathcal{E}^{t}\right)_{A B} d y^{A} \wedge d y^{B} \equiv \Omega_{A B} d y^{A} \wedge d y^{B}
$$

which is preserved by the Ricci flow of the fiber metric

$$
\mathcal{S}^{-1} \partial_{t} \mathcal{S}=D^{i}\left(\mathcal{S}^{-1} \partial_{i} \mathcal{S}\right)
$$

The Ricci flow preserves the form (7.5) of the $3 \mathrm{~d}$ target metric with $\mathcal{S}_{A B}$ a positive symmetric matrix in $\operatorname{Sp}(2 h, \mathbb{R})$. Thus the Ricci flow for the metric (7.5) evolves the complex moduli of the Abelian fibers of $\mathscr{X} \rightarrow \mathscr{M}$ but not their Kähler moduli. At a weakly-coupled fixed-point of the $2 \mathrm{~d}$ RG flow the tension $D^{i}\left(\mathcal{S}^{-1} \partial_{i} \mathcal{S}\right)$ vanishes and hence the fixed-point map $\tilde{\mu}$ is harmonic.

\subsubsection{Naturalness of the effective Lagrangian $\mathscr{L}_{\text {eff }}$}

In terms of the original $4 \mathrm{~d}$ effective theory (7.4) the vanishing of the tension $T(\mu)$ means that the two ${ }^{49}$ (quadratically divergent) one-loop Feymann graphs in figure 1 cancel. Indeed the leading correction to gauge couplings is proportional to the tension of the gauge coupling

$$
\delta \tau_{a b} \propto \frac{\Lambda_{\text {eff }}^{2}}{F_{\pi}^{2}} T(\mu)_{a b} \equiv \frac{\Lambda_{\text {eff }}^{2}}{F_{\pi}^{2}} D^{i} \partial_{i} \tau_{a b}
$$

In order for the $4 \mathrm{~d}$ Lagrangian (7.4) to be meaningful as a weakly-coupled effective description of the low-energy dynamics, the correction (7.11) should be rather small. If $F_{\pi} \lesssim \Lambda_{\text {eff }}$, the tension should be approximately zero $T(\mu)_{a b} \approx 0$. Thus the vanishing of the tension may be seen as a "naturalness" requirement for the weakly-coupled $4 \mathrm{~d}$ effective theory $\mathscr{L}_{\text {eff. }}$ This is equivalent to the statement that the gauge coupling $\mu$ is (approximately) harmonic, i.e. that its energy is near the minimum value ${ }^{50}$ consistent with the monodromy representation $\rho$.

The interpretation of $T(\mu)_{a b}=0$ as a naturalness condition on $\mathscr{L}_{\text {eff }}$ is reflected in the fact that the gauge coupling tension vanishes for all $4 \mathrm{~d}$ supersymmetric theories whether they are rigid SUSY QFTs or supergravities. This is one of the many ways in which SUSY improves naturalness. At weak coupling, naturalness of the gauge couplings does not require $\tau(\phi)_{a b}$ to satisfy all the detailed constraints of supersymmetry: the much

\footnotetext{
${ }^{49}$ There is a third one-loop graph (the tadpole graph) proportional to the Christoffel symbols $\gamma_{j k}^{i}$. The tadpole graph may be set to zero by using normal coordinates in the perturbative expansion.

${ }^{50}$ Assuming our working hypothesis that the energy of $\mu$ is finite.
} 

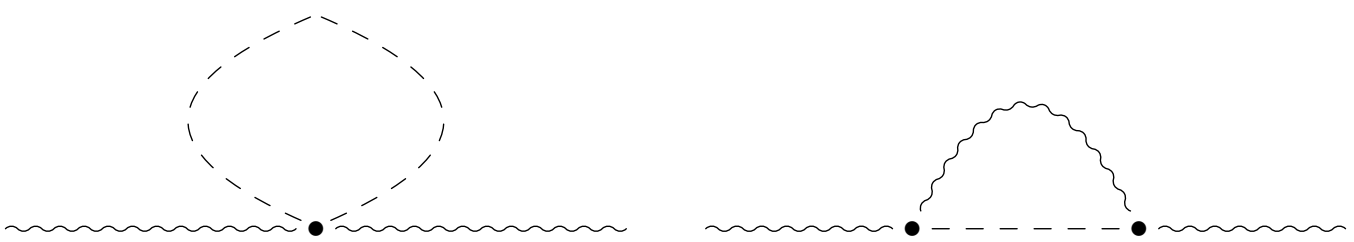

Figure 1. One-loop corrections to the photon propagator arising from the scalar-vector couplings in the vectors' kinetic terms. Wavy lines are photons, dashed ones scalars.

weaker condition that the gauge coupling map $\mu$ is harmonic suffices. When $\mathscr{M}$ is an OV manifold and $\mu$ has finite energy, the weaker condition implies that the gauge coupling $\mu$ is tamed and arithmetic; whenever the holonomy of the OV manifold is not generic, we either get an arithmetic $t t^{*}$ geometry or an arithmetic domestic geometry of the kind we have in extended supergravity. ${ }^{51}$ In other words, when $\mathscr{M}$ is an OV manifold with a sufficiently large algebra $\mathcal{P}^{\bullet}$, the weaker naturalness condition on $\mu$ is equivalent to the detailed constraints from supersymmetry.

Let $\mu: \mathscr{M} \rightarrow \Lambda \backslash \operatorname{Sp}(2 h, \mathbb{R}) / \mathrm{U}(h)$ be the gauge coupling of an effective theory $\mathscr{L}_{\text {eff, }}$ with $\mathcal{M}$ an OV space. Under our working hypothesis that $\mu$ has finite energy, the homotopy class $[\mu]$ contains a unique tensionless map $\stackrel{\mu}{\mu}$, which is automatically tamed. The naturalness argument above suggests that the torsion $T(\mu)$, and hence the correction $\mu-\stackrel{\circ}{\mu}$, is small, that is, that domestic geometry is (at least) approximatively correct.

\subsubsection{Naturalness: adding gravity}

We add gravity to the model (7.4), and return to the original Lagrangian (2.1) coupled to gravity, and again compactify the model on $S^{1}$. In a gravity theory this is just a topological sector of the $4 \mathrm{~d}$ theory, and the $3 \mathrm{~d}$ effective low-energy physics ought to be quantum-consistent if the $4 \mathrm{~d}$ theory is. In presence of gravity the $3 \mathrm{~d}$ effective theory has two additional light scalars: $\rho$ corresponding to $g_{44}$ and the dual $z$ of the KK vector $g_{\mu 4}$. The 3 d scalars' manifold $\mathscr{M}_{3}$ is now a fibration over $\mathscr{M} \times \mathbb{R}_{\rho}$ with fiber a copy of the locally homogeneous space $H(\mathbb{Z}) \backslash H(\mathbb{R})$ where $H(\mathbb{R})$ is the real Heisenberg Lie group

$$
H(\mathbb{R})=\left\{\left(z, y^{A}\right) \in \mathbb{R}^{2 h+1}\right\} \quad\left(z, y^{A}\right) \cdot\left(w, u^{A}\right)=\left(z+w+\Omega_{A B} y^{A} u^{B}, y^{A}+u^{A}\right) .
$$

Ignoring quantum corrections (which are supressed as $\rho \rightarrow \infty$ ), the Einstein frame $3 \mathrm{~d}$ scalars' metric takes the form

$$
\begin{aligned}
d s^{2} & =d s_{\text {base }}^{2}+d s_{\text {fiber }}^{2}= \\
& =\left(G_{i j} d x^{i} d x^{j}+\frac{1}{2 \rho^{2}} d \rho^{2}\right)+\frac{1}{2 \rho^{2}}\left(2 \rho \mathcal{S}_{A B} d y^{A} d y^{B}+\left(d z+\Omega_{A B} y^{A} d y^{B}\right)^{2}\right),
\end{aligned}
$$

\footnotetext{
${ }^{51}$ That is, a totally geodesic embedding into the locally symmetric space $\Lambda \backslash G(\mathbb{R}) / K$.
} 
with $G_{i j}, \mathcal{S}_{A B}$ and $\Omega_{A B}$ as before. The Killing vector $\partial_{z}$ defines on each fiber a transversely holomorphic foliation (THF) ${ }^{52}$ endowed with a THF-compatible transversely Kähler metric: the normal bundle to the leaves of the transversely holomorphic foliation carries a complex structure as well as the transverse Hermitian metric $\rho^{-1} \mathcal{S}_{A B} d y^{A} d y^{B}$ which is Kähler when restricted to a fiber with Kähler form $\rho^{-1} \Omega_{A B} d y^{A} \wedge d y^{B}$ (cfr. eq. (7.9)). The complex structure of the normal bundle to the THF foliation on each fiber is again specified by the uni-modular matrix $\mathcal{S}_{A B}$.

In view of the Heisenberg symmetry and the scaling invariance $\left(\rho, z, y^{A}\right) \rightarrow\left(\lambda^{2} \rho, \lambda^{2} z, \lambda y^{A}\right)$, the components of the Ricci tensor along the fiber must have the form

$$
R_{A B} d y^{A} d y^{B}+R_{z z} d z^{2}=\frac{1}{\rho} A_{A B} d y^{A} d y^{B}+\frac{1}{\rho^{2}} B\left(d z^{2}+\Omega_{A B} y^{A} d y^{B}\right)^{2}
$$

where $A_{A B}$ and $B$ are functions only of the coordinates $x^{i}$ of $\mathscr{M}$. In particular the Ricci flow preserves the THF of each fiber, while evolves its transverse complex structure and Kähler form

$$
\frac{d}{d t}\left(\frac{1}{\rho} \mathcal{S}_{A B}\right)=-\frac{2}{\rho} A_{A B}
$$

where

$$
A=-\frac{1}{2} \mathcal{S}\left(D^{i}\left(\mathcal{S}^{-1} \partial_{i} \mathcal{S}\right)-c \mathbf{1}\right)
$$

for some constant $c$ which depends only on $h$ and $m$. Writing $\mathcal{S}=f \mathcal{S}^{\prime}$, with $\operatorname{det} \mathcal{S}^{\prime}=1$, we get for the uni-modular matrix $\mathcal{S}^{\prime}$ the same flow equation as before, eq. (7.10), so the evolution of the transverse complex structure of the fiber-wise THF is again given by the $4 \mathrm{~d}$ tension flow. The new aspect is that now the transverse Kähler form gets rescaled by the factor $e^{c t}$. This is due to the fact that gravity modifies the Ricci flow equation so that the appropriate 3d "fixed point condition" requires $\mathscr{M}_{3}$ to be Einstein rather than Ricci-flat; the easiest way to see this is to compare $3 \mathrm{~d} \mathcal{N} \geq 3$ rigid SUSY field theories, which have Ricci-flat target spaces, with the $\mathcal{N} \geq 3$ supergravities which have Einstein target spaces with $R_{i j}=-\lambda g_{i j}$, where $\lambda>0$ is a universal constant which depends only on the field content. In "natural" effective theories the additional term proportional to $\mathcal{S}_{A B} / \rho$ in the r.h.s. of eq. (7.15) should be cancelled by diagrams of the form in figure 1 with the scalar internal lines replaced by graviton propagators whose contribution is $\propto \mathcal{S}_{A B} / \rho$. Thus the absence of torsion of $\mu$ can again be interpreted as a naturalness condition on the $4 \mathrm{~d}$ effective gravity theory. All weakly-coupled infrared fixed points then have tensionless gauge couplings $\mu$.

In presence of fermions, the quadratic divergence of the photon propagators, in addition to the diagrams in figure 1 , gets contributions from one-loop of fermions

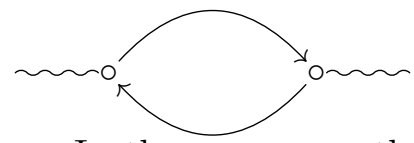

where $\circ$ stands for Pauli coupling. In the SUSY case these new contributions would not change our conclusions because of the relation of Pauli couplings to the gauge couplings

\footnotetext{
${ }^{52}$ Probably the best reference for transversely holomorphic foliations (THF) is the physics paper [95] which discuss them in the context of supersymmetry on curved $3 \mathrm{~d}$ manifolds. They discuss the $3 \mathrm{~d}$ case; the extension to an arbitrary odd number of dimensions is straightforward.
} 
implied by supersymmetry: the net effect is just a further contribution to the constant $c$ in (7.16), that is, the Pauli couplings will not modify the flow of the fiber's transverse complex structure. It seems plausible that this conclusion remains valid in all consistent effective theories, possibly with more complicate flows for the fiber's transverse Kähler moduli, but without affecting the flow of the fiber's transverse complex moduli. If this is the case, the "natural" value of the tension of the gauge coupling is still zero (or very small). We expect the fiber's flow to have this property for the following reason: according to the definition of OV manifold, $\pi_{1}(\mathscr{M})$ is "big" and — unless $\mu$ is the constant map" ${ }^{53}$ - its image $\Gamma$ in the Siegel modular group should also be "big" ${ }^{54}$ Since $\Gamma$ is a gauge symmetry, the Pauli couplings should be exactly invariant under it. In the extreme IR this implies invariance under the continuous group $\operatorname{Sp}(2 h, \mathbb{R})$. Defining (as it is usual) the fermi fields to be invariant under $\Gamma$, the electro-magnetic field strengths ${ }^{55} \mathcal{F} \equiv\left(G_{a}, F^{a}\right)$ can enter the Pauli interactions only through the $\Gamma$-invariant combination $\mathcal{E}^{-1} \mathcal{F}$ : these couplings are automatically $\operatorname{Sp}(2 h, \mathbb{R})$-invariant and hence cannot spoil the tension flow of the uni-modular matrix $\mathcal{S}_{a b}^{\prime}$ which is a flow in the group $\operatorname{Sp}(2 h, \mathbb{R})$. In particular, when $\mu$ is harmonic, hence tamed, the Pauli coupling must be a domestic brane amplitude, as expected.

More generally, the tension field in the r.h.s. of eq. (7.2) is the only $\operatorname{Sp}(2 h, \mathbb{R})$-covariant tensor we can write to second order in the scalar field derivatives which flows the transverse complex structure of the Heisenberg fiber of $\mathscr{M}_{3}$; all possible covariant corrections (to this order) will affect the fiber's transverse Kähler moduli but not its transverse complex moduli.

Therefore the condition that $\mu$ is harmonic seems to be a rather plausible "naturalness" requirement, at least under certain circumstances, especially when the scalars' manifold $\mathscr{M}$ is an OV space. If the couplings flow in the extreme IR to a "weak-coupling" regime (meaning a regime in which the geometric interpretation of the couplings is sound), at the fixed point the gauge coupling $\mu$ should be tensionless, i.e. $\mu$ harmonic. As already stressed, we know no reliable example of consistent effective theory where the tension of $\mu$ is not zero.

\subsubsection{A word of caution}

In the heuristics above we neglected the issues related to finite-distance singularities in scalars' space. At such points finitely many charged states become light, and we have additional effective one-loop contributions from diagrams of the form (7.17) where the internal lines correspond to the new light charged particles and the vertices $\circ$ are minimal gauge couplings. In terms of the dependence on the effective cut-off $\Lambda_{\text {eff }}$, these contributions are suppressed by a factor $\Lambda_{\text {eff }}^{-2} \log \Lambda_{\text {eff }}$, but there is no reason to expect that - as functions of the background value of the scalars - they are proportional to the tension of the gauge

\footnotetext{
${ }^{53}$ This exceptional case does happen in quantum-consistent theories: consider the $4 \mathrm{~d} \mathcal{N}=2$ SUGRA which describes Type IIB on a rigid Calabi-Yau 3-fold.

${ }^{54}$ Assuming $\mu$ not to be the constant map (which is a special case of harmonic map), the technical statement is that $\Gamma$ is Zariski dense in some non-compact Lie subgroup $G(\mathbb{R}) \subset \operatorname{Sp}(2 h, \mathbb{R})$ : see section 7.3. This means that all algebraic invariants for the group $\Gamma$ are invariants for the full group $G(\mathbb{R})$, which generically is the full group $\operatorname{Sp}(2 h, \mathbb{R})$.

${ }^{55}$ The dual field strengths $G_{a}$ are defined as $G_{a}=* \partial \mathscr{L} / \partial F^{a}$.
} 
couplings $\mu$. This suggests that near the finite-distance singular points in $\mathscr{M}$ the effective tension $\left(\Lambda_{\text {eff }} / F_{\pi}\right)^{2} T(\mu)_{a b}$, or more precisely its invariant norm $\left(\Lambda_{\text {eff }} / F_{\pi}\right)^{2}\left\|T(\mu)_{a b}\right\|$, is small but non-zero. Morally speaking, we expect the gauge couplings $\tau(\phi)_{a b}$ to satisfy some kind of "wave-equation with sources" on $\mathscr{M}$ roughly of the form

$$
T(\mu)_{a b} \equiv D^{i} \partial_{i} \tau(\phi)_{a b}=\sum_{s} f_{s}\left(\phi ; \phi_{s}\right)
$$

where $\phi_{s} \in \mathscr{M}$ are the finite-distance singular points at which finitely-many new degrees of freedom become massless, and the $f_{s}\left(\phi ; \phi_{s}\right)$ 's are functions (or distributions) with support in some small region centered at $\phi_{s}$ which capture the local physics at these special points in moduli space.

\subsection{Existence, uniqueness, and structure of tamed maps}

In an $\mathcal{N}=2$ supergravity consistent with the swampland conjectures, the gauge coupling map

$$
\mu: \mathscr{M} \rightarrow \Lambda \backslash \operatorname{Sp}(2 h, \mathbb{R}) / \mathrm{U}(h), \quad \Lambda \subseteq \mathrm{Sp}(2 h, \mathbb{Z}),
$$

has a very restricted form [15] as a consequence of the structure theorem for the underlying VHS period map [58-60]. It is natural to ask whether this fundamental result (and its physical consequences [15]) holds in the more general set-up of domestic geometry, that is, for finite-energy tamed maps $\mu$ as in eq. (7.19) whose source $\mathscr{M}$ is an OV manifold.

The aim of this subsection is to argue for a positive answer. For didactical reasons we split the discussion in two parts: first we consider the well studied case where the source of the map $\mu$ is a compact Riemannian manifold (whose holonomy algebra may or may not be special). This situation has little interest for physics, however it sheds light on the basic structures of domestic geometry. Then we proceed to the physically relevant set-up of non-compact OV manifolds, and argue that the good properties of the compact case extends to domestic geometry defined over the "magic" OV spaces.

We apologize for being technical if not pedantic. The reader may prefer to jump directly to section 7.3.3 in a first reading. However some intermediate result may be of independent interest.

\subsubsection{Compact source space}

The argument goes through several steps. First we show that twisted tamed maps (7.19) with the required properties exist under the assumptions:

(i) the source manifold is compact;

(ii) the monodromy representation has the physically expected properties.

Since a tamed map is in particular harmonic, this step requires first to show that twisted harmonic maps exist, and then that they are tamed. The second step concerns the uniqueness properties of twisted tamed maps with a given monodromy representation. In the third step we use this information to get the structural factorization of the domestic geometry. This last property coincides with the VHS structure theorem whenever the source manifold is Kähler. 
Existence of harmonic maps. The most classical existence theorem for harmonic maps is due to Eells and Sampson [87]; their strategy was to show existence and regularity of the tension flow (7.1): let $M$ and $N$ be compact Riemannian manifolds and $N$ have non-positive sectional curvatures. Then every smooth map $f: M \rightarrow N$ is homotopic to a harmonic map. The harmonic map is essentially unique in its homotopy class ${ }^{56}$ and it is the map of minimal energy in its class.

For the gauge coupling map (7.19) the condition on the sectional curvatures of the target space is satisfied, but both the source and the target manifolds are non-compact. However they are expected to behave "almost as they were compact" because they have finite volume and enjoy other good properties. Thanks to these special properties, we may invoke other, more powerful, existence theorems.

Our target space is locally symmetric; in this situation one has:

Theorem (Corlette [88]). Let $G$ be a real Lie group, $K \subset G$ a maximal compact subgroup, and $\Lambda \subset G$ any discrete subgroup. Assume the Riemann manifold $M$ is compact. A harmonic map

$$
\phi: M \rightarrow \Lambda \backslash G / K
$$

exists if and only if the monodromy group $\rho\left(\pi_{1}(M)\right) \equiv \Gamma \subseteq \Lambda$ has reductive Zariski closure ${ }^{57}$ $\bar{\Gamma}^{\mathbb{R}}$ in $G$.

In our problem, eq. (7.19), the target space has the required form with

$$
G=\operatorname{Sp}(2 h, \mathbb{R}), \quad K=\mathrm{U}(h),
$$

while we may take $\Lambda \equiv \Gamma$ and neat with no loss. In the applications we have in mind the real Lie group ${ }^{58} G^{\prime} \equiv \bar{\Gamma}^{\mathbb{R}} \subset G$ - if non-trivial — is semi-simple and non-compact: these properties follow from the swampland conditions on the monodromy. ${ }^{59}$ In view of the above theorem, these "swampy" properties of $\Gamma$ guarantee the existence of a twisted harmonic map $\phi$.

The maps of domestic geometry are not just harmonic, they are tamed. We turn to the question of tameness of the harmonic map $\phi$.

The map $\phi$ is automatically tamed. When the compact manifold $M$ has special holonomy $\mathfrak{h o l}(M) \neq \mathfrak{s o}(m)$ - and hence a non-trivial algebra of parallel forms $\mathcal{P}^{\bullet}$ - the twisted harmonic map $\phi$ is automatically tamed, that is,

$$
D *(d \phi \wedge \Omega)=0 \quad \text { for all } \quad \Omega \in \mathcal{P}^{\bullet}
$$

(see [69], theorem 3.1). In particular

(a) $M$ is Kähler $\Rightarrow \phi$ is pluriharmonic $\Rightarrow \phi$ defines a $t t^{*}$ geometry (cfr. §. (5.2));

\footnotetext{
${ }^{56}$ For the precise uniqueness statement see e.g. [93].

${ }^{57}$ We see the Lie group as an algebraic group over $\mathbb{R}$ through its adjoint representation.

${ }^{58}$ More correctly: the real Lie group underlying the $\mathbb{R}$-algebraic group $\bar{\Gamma}^{\mathbb{R}}[96]$.

${ }^{59} \mathrm{By}$ construction, in the relevant applications the group $\Gamma$ is a finite-index, neat, normal subgroup of the physical monodromy group.
} 
(b) $M$ quaternionic Kähler of dimension $\geq 8$, or symmetric not of the form $\mathrm{SO}(n, 1) / \mathrm{SO}(n)$ or $\mathrm{SU}(m, 1) / \mathrm{U}(m) \Rightarrow \phi$ is totally geodesic, so its image is either a point or a locally symmetric space of the form $\Gamma^{\prime} \backslash L / K^{\prime}$ for $L \subset G$ a Lie subgroup [67].

The proof of eq. (7.22) is via a Bochner argument [69] which generalizes the one based on eq. (5.16) which was valid under the assumption that $M$ is Kähler. If $\phi$ is harmonic and the target space has non-positive curvature operators (as in our case) one has an identity of the form [69]

$$
\begin{aligned}
& d\left(*\left(d \phi^{i} \wedge \Omega\right) \wedge D *\left(d \phi^{j} \wedge * \Omega\right) g_{i j}\right)= \\
& \quad=(-1)^{m-1}\left(|D *(d \phi \wedge \Omega)|^{2}+\text { non-negative }\right) d \text { vol. }
\end{aligned}
$$

For $M$ compact the integral of the l.h.s. vanishes, so $D *(d \phi \wedge \Omega)=0$ and the map is tamed.

Uniqueness properties $I$. One expects that the very same "swampy" conditions on $\Gamma$ which guarantee the existence of the tamed map $\phi$ also play the crucial role in the question of essential uniqueness of the tamed map $\phi$ in its homotopy class (i.e. in the family of twisted maps with the given monodromy representation $\rho$ ). Here the main theme is to qualify the adjective "essential", that is, to specify under which equivalence relation(s) all homotopic tamed maps get identified: the coarser the relation, the weaker the uniqueness property. The weakest result is:

The "swampy" properties of $\Gamma$ imply that the space of tamed maps in the class of $\phi$ is homotopic to a single point. ${ }^{60,61}$

We look for a much stronger result: uniqueness up to the physically natural equivalence relation (see definition 7 below). We start by playing the devil's advocate, and see what happens when the harmonic map is not unique. The following argument is modelled on the classical papers [92, 93]; in facts it reproduces the main points in the proof of theorem 9.7.2 of ref. [91] whose hypotheses are identical to ours: (i) compact source space, and (ii) target space a complete Riemannian manifold with non-positive sectional curvatures. (We go through the proof because its single steps are more useful for our purposes than the theorem itself.)

\footnotetext{
${ }^{60}$ We sketch the argument. Let $N$ be any Riemannian manifold (not necessarily compact) with $\pi_{1}(N) \equiv \pi$ and let $\mathfrak{M}(N, \Gamma \backslash G / K)_{f}$ be the space of $C^{2}$-maps $N \rightarrow \Gamma \backslash G / K$ homotopic to the map $f\left(\mathfrak{M}(N, \Lambda \backslash G / K)_{f}\right.$ is endowed with the $C^{2}$-topology). By Gottlieb lemma $[89,90] \mathfrak{M}(N, \Gamma \backslash G / K)_{f}$ is a $K\left(C_{\pi, f}, 1\right)$ space with $C_{\pi, f}$ the centralizer of $f_{*}(\pi)$ in $\Gamma$. Under our "swampy" assumptions $C_{\pi, f}$ is trivial, and $\mathfrak{M}(N, \Gamma \backslash G / K)_{f}$ has the homotopy type of a point. We can compute the homotopy type of $\mathfrak{M}(N, \Gamma \backslash G / K)_{f}$ by Morse cobordisim applied to the gradient flow of the energy $E(\phi)$ (i.e. to the tension flow). Under the present assumptions the energy $E(\phi)$ is a "perfect Morse function" in the sense that its Hessian at a critical point is a non-negative operator: see e.g. corollary 9.2.2 of [91]. Then we conclude that the space of harmonic functions homotopic to $f$ is contractible.

${ }^{61}$ If the condition of non-positive sectional curvatures is replaced by the stronger one of strictly negative sectional curvatures, the space of tamed maps is a single point and not just homotopic to a point [93]. In the physical set-up this applies only when we have a single light photon i.e. $h=1$.
} 
Let $\phi_{0}, \phi_{1}$ be two distinct homotopic harmonic maps; one can construct a homotopy

$$
\phi(x, t): M \times[0,1] \rightarrow \Gamma \backslash G / K, \quad\left[\begin{array}{l}
\phi(x, 0)=\phi_{0}(x) \\
\phi(x, 1)=\phi_{1}(x)
\end{array}\right.
$$

which is geodesic, i.e. for fixed $x \in M$ the map

$$
\phi(x,-):[0,1] \rightarrow \Gamma \backslash G / K
$$

is a geodesic. We write $E(t)$ for the energy of the map $\phi(-, t): M \rightarrow \Gamma \backslash G / K$. A simple computation yields ${ }^{62}$

$$
\frac{\partial^{2} E(t)}{\partial t^{2}}=\int_{M} d \operatorname{vol}\left(\left\|\nabla_{\partial / \partial t} d \phi(t)\right\|^{2}-g^{\alpha \beta} G_{i j} R_{i j k l} \partial_{t} \phi^{i} \partial_{\alpha} \phi^{j} \partial_{t} \phi^{k} \partial_{\beta} \phi^{l}\right)
$$

which, together with the vanishing of the first variation at a harmonic map

$$
\left.\frac{\partial E(t)}{\partial t}\right|_{t=0}=0
$$

yields

$$
\begin{aligned}
& E(\phi(t))-E\left(\phi_{0}\right)= \\
& =\int_{0}^{t} d s \int_{0}^{s} d u \int_{M} d \operatorname{vol}\left(\left\|\nabla_{\partial / \partial u} d \phi(u)\right\|^{2}-g^{\alpha \beta} G_{i j} R_{i j k l} \partial_{u} \phi^{i} \partial_{\alpha} \phi^{j} \partial_{u} \phi^{k} \partial_{\beta} \phi^{l}\right) \geq 0,
\end{aligned}
$$

where the inequality holds because the target space sectional curvatures are non-positive. Setting $t=1$ we get $E\left(\phi_{1}\right) \geq E\left(\phi_{0}\right)$; inverting the rôle of the two maps we get $E\left(\phi_{1}\right)=$ $E\left(\phi_{0}\right)$, so all homotopic harmonic maps have the same energy $E\left(\phi_{0}\right)$, and hence they all realize the absolute minimum of the energy in their class. Then each of the two terms inside the inner integral in the r.h.s. of (7.28) is point-wise zero for all $u$ 's. We conclude that

$$
E(\phi(t))-E\left(\phi_{0}\right)=0 \text { for all } t
$$

and thus all maps $\phi(t)(t \in[0,1])$ have the same minimal value $E\left(\phi_{0}\right)$ of the energy. From the variational charaterization of harmonic maps we get:

Proposition 5 (theorem 9.7.2 of ref. [91]). The maps $\phi(-, t): M \rightarrow \Gamma \backslash G / K$ form $a$ one-parameter family of homotopic harmonic (hence tamed) maps.

A harmonic map $\phi$ is a stationary classical soliton of the $\sigma$-model with target $\Gamma \backslash G / K$ defined in the "space-time" $\mathbb{R}_{\text {time }} \times M$ whose mass is $E(\phi)$. Proposition 5 says that whenever the twisted tamed map $\phi$ is not unique in its class, we have a continuous family of $\sigma$ model solitons which are degenerated in mass and carry the same topological charges. As physicists we do not expect such a huge degeneracy, unless it is a consequence of a symmetry of the $\sigma$-model. We make this physical intuition into a math definition:

\footnotetext{
${ }^{62}$ See corollary 9.2.1 in [91]. For later reference we stress that equation (7.26) holds under the assumption that $\phi(t)$ is a geodesic family of finite-energy maps (not necessarily harmonic) independently of the assumption that the source space $M$ is compact (cfr. ref. [91]).
} 
Definition 7. We say that the tamed map $\phi: M \rightarrow \Gamma \backslash G / K$ is essentially unique in its homotopy class iff all tamed maps homotopic to it are obtained from $\phi$ by the action of a symmetry of the associated $\sigma$-model.

We expect the tamed map to be essentially unique in this precise sense. This physical intuition turns out to be fully correct: see lemma 4 below. As a preliminary step we need to discuss the way symmetry acts.

Action of symmetry. The subgroup of the $\sigma$-model symmetry which leaves invariant the monodromy representation $\rho$ - that is, which commutes with the topological charges is the centralizer $C \subset G$ of $\Gamma$. $C$ is a real Lie group ${ }^{63}$ identified with the centralizer of $G^{\prime} \subset G$ (where, as before, $G^{\prime} \equiv \bar{\Gamma}^{\mathbb{R}}$ ). When $G^{\prime}$ is reductive (as required for $\phi$ to exists) but not necessarily semi-simple

$$
G^{\prime}=Z\left(G^{\prime}\right) \times G_{s s}^{\prime}, \quad G_{s s}^{\prime} \text { semi-simple }
$$

$C$ is the center $Z\left(G^{\prime}\right)$ of $G^{\prime}$ times the Lie group $G^{\prime \prime}$ such that $G^{\prime} \times G^{\prime \prime}$ is a maximal subgroup of $G$ :

$$
C=Z\left(G^{\prime}\right) \times G^{\prime \prime}, \quad G^{\prime} \times G^{\prime \prime} \hookrightarrow G \text { maximal }
$$

Let $K^{\prime} \subset G^{\prime}, K^{\prime \prime} \subset G^{\prime \prime}$ be maximal compact subgroups, and $K \subset G$ a maximal compact subgroup containing $K^{\prime} \times K^{\prime \prime}$. We have a chain of totally geodesic embeddings

$$
\Gamma \backslash G^{\prime} / K^{\prime} \stackrel{\iota_{2}}{\longrightarrow} \Gamma \backslash G^{\prime} / K^{\prime} \times G^{\prime \prime} / K^{\prime \prime} \stackrel{\iota_{1}}{\longrightarrow} \Gamma \backslash G / K .
$$

Since $\Gamma$ is neat, by the Cartan-Hadamard theorem all three locally symmetric manifolds in eq. (7.32) are $K(\Gamma, 1)$ spaces; then by the Whitehead theorem [97] there exists a chain of deformation retractions

$$
\Gamma \backslash G / K \stackrel{r_{1}}{\longrightarrow} \Gamma \backslash G^{\prime} / K^{\prime} X G^{\prime \prime} / K^{\prime \prime} \stackrel{r_{2}}{\longrightarrow} \Gamma \backslash G^{\prime} / K^{\prime} .
$$

Let

$$
\phi: M \rightarrow \Gamma \backslash G / K
$$

be a twisted tamed map with monodromy representation $\rho$. We consider the two maps

$$
f_{1} \equiv r_{1} \circ \phi: M \rightarrow \Gamma \backslash G^{\prime} / K^{\prime} \backslash G^{\prime \prime} / K^{\prime \prime}, \quad f_{2} \equiv r_{2} \circ r_{1} \circ \phi: M \rightarrow \Gamma \backslash G^{\prime} / K^{\prime},
$$

which (by construction) are twisted by the same $\rho$. By theorem (7.20) there exist tamed maps $\phi_{1}$ and $\phi_{2}$ homotopic (respectively) to $f_{1}, f_{2}$. Since $\iota_{1}, \iota_{2}$ are totally geodesic, we obtain three tamed maps $M \rightarrow \Gamma \backslash G / K$ twisted by the same $\rho$

$$
\phi, \quad \iota_{1} \phi_{1}, \quad \iota_{1} \iota_{2} \phi_{2},
$$

where the second (third) is a tamed map whose image is fully contained in the submanifold $\Gamma \backslash G^{\prime} / K^{\prime} \times G^{\prime \prime} / K^{\prime \prime}$ (resp. $\Gamma \backslash G^{\prime} / K^{\prime}$ ) of $\Gamma \backslash G / K$. By its very definition the third tamed map factorizes as

$$
M \stackrel{\iota_{2} \phi_{2}}{\longrightarrow} \Gamma \backslash G^{\prime} / K^{\prime} \backslash G^{\prime \prime} / K^{\prime \prime} \stackrel{\iota_{1}}{\longrightarrow} \Gamma \backslash G / K,
$$

\footnotetext{
${ }^{63}$ Indeed an algebraic group over $\mathbb{R}$.
} 
while the projection of the first arrow $\iota_{2} \phi_{2}$ in the factor space $G^{\prime \prime} / K^{\prime \prime}$ is a constant map. The other projection is just the tamed map

$$
M \stackrel{\phi_{2}}{\longrightarrow} G^{\prime} / K^{\prime} \equiv Z\left(G^{\prime}\right) /\left[K^{\prime} \cap Z\left(G^{\prime}\right)\right] \times G_{s s}^{\prime} /\left[K^{\prime} \cap G_{s s}^{\prime}\right]
$$

which is identified with a pair of solitons for the two $\sigma$-models with respective target spaces the two factors in the r.h.s. . The first factor space is a locally flat Abelian group $A$. The "swampy" conditions on $\Gamma$ say that this Abelian factor is absent. For $\Gamma$ just reductive, the $\sigma$-model soliton (7.37) decomposes in three items: (i) a constant map into $G^{\prime \prime} / K^{\prime \prime}$, (ii) a soliton of the $\sigma$-model with target $A$, and (iii) a soliton of the $\sigma$-model with target the semi-simple part of the double coset $\Gamma \backslash G^{\prime} / K^{\prime}$.

The symmetry $Z\left(G^{\prime}\right) \times G^{\prime \prime}$ acts on the $\sigma$-model soliton (7.37) by translations of the image of the constant map in $G^{\prime \prime} / K^{\prime \prime}$ and shift symmetries of the Abelian soliton. ${ }^{64}$ In the "swampy" case the Abelian sector is absent, and the symmetry produces out of the solution (7.37) a $G^{\prime \prime} / K^{\prime \prime}$-family of harmonic solitons. Proposition 5 requires this family to decompose into geodesic sub-families; they have the form

$$
M \times[0,1] \stackrel{\phi_{2} \times \lambda}{\longrightarrow} \Gamma \backslash G^{\prime} / K^{\prime} \times G^{\prime \prime} / K^{\prime \prime} \stackrel{\iota_{1}}{\longrightarrow} \Gamma \backslash G / K
$$

with $\lambda:[0,1] \rightarrow G^{\prime \prime} / K^{\prime \prime}$ a geodesic arc connecting two points in $G^{\prime \prime} / K^{\prime \prime}$.

Uniqueness properties II. We have

Lemma 4. $M$ compact. Suppose $\Gamma$ satisfies the "swampy" conditions, i.e. $G^{\prime} \equiv \bar{\Gamma}^{\mathbb{R}}$ is a non-compact semi-simple algebraic subgroup of $G$. Let

$$
\phi_{0}, \phi_{1}: M \rightarrow \Gamma \backslash G / K
$$

be two twisted harmonic (hence tamed) maps in the same homotopy class (三 twisted by the same monodromy representation $\rho$ ). Then there is an element $g \in G^{\prime \prime}$ of the $\sigma$-model symmetry group such that

$$
\phi_{1}=g \cdot \phi_{0} .
$$

Since we have already proven that in the homotopy class of $\phi_{0}, \phi_{1}$ there is at least one $G^{\prime \prime} / K^{\prime \prime}$-family of tamed maps generated by a factorized tamed map

$$
M \stackrel{\phi_{*}}{\longrightarrow} \Gamma \backslash G^{\prime} / K^{\prime} \stackrel{\iota_{1} \iota_{2}}{\longrightarrow} \Gamma \backslash G / K,
$$

while this family form a full $G^{\prime \prime}$-orbit, we conclude

Corollary 1. Under the assumptions of the lemma, the set of tamed maps

$$
\phi: M \rightarrow \Gamma \backslash G / K
$$

twisted by $\rho$ is given by a unique $G^{\prime \prime} / K^{\prime \prime}$-family of maps factorized as in eq. (7.37). In particular $\phi$ is essentially unique in its homotopy class in the sense of definition 7 .

\footnotetext{
${ }^{64}$ The fundamental group of the family of solitons produced by acting on (7.37) with the symmetry group is $\Gamma \cap Z\left(G^{\prime}\right)$, so that this family is homotopic to the space of all solitons with target space $\Gamma \backslash G / K$, cfr. footnote 60 .
} 
Proof of the lemma. Preliminary we recall from the previous sections some facts about the non-compact symmetric space $G / K$. Let $\mathfrak{g}=\mathfrak{k} \oplus \mathfrak{p}$ be the orthogonal decomposition of the Lie algebra of $G$ (with $\mathfrak{k} \equiv \mathfrak{L} \mathfrak{i}(K)$ ). The tangent bundle $T(G / K)$ is the homogeneous bundle associated to the $K$-module $\mathfrak{p}$. We choose a global section $s: G / K \rightarrow G$ (it exists since $G / K$ is contractible). The Levi-Civita connection of $G / K$ (in the trivialization of $T(G / K)$ defined by the chosen $s)$ is

$$
\nabla=d+\left(s^{-1} d s\right)_{\mathfrak{k}}
$$

where as before the subscript means orthogonal projection to the subspace $\mathfrak{k} \subset \mathfrak{g}$. Then

$$
-R_{i j k l} v^{i} w^{j} v^{k} w^{l}=\left\|\left[\iota_{v}\left(s^{-1} d s\right)_{\mathfrak{p}}, \iota_{w}\left(s^{-1} d s\right)_{\mathfrak{p}}\right]\right\|^{2} \geq 0 .
$$

Now let $\phi_{0}, \phi_{1}: M \rightarrow \Gamma \backslash G / K$ be two tamed maps twisted by the same $\rho$. We write $\phi_{t} \equiv$ $\phi(-, t)$ for the geodesic family of interpolating tamed maps in proposition 5 , parametrized by $t \in[0,1]$, and consider its lift to the universal cover $\tilde{M}$ of $M$

$$
\tilde{\phi}_{t}: \tilde{M} \times[0,1] \rightarrow G / K
$$

$\tilde{\phi}_{t}$ is $\rho$-twisted, that is, if $\pi_{1}(M) \ni \xi: \tilde{M} \rightarrow \tilde{M}$ is an element of the deck group of the cover $\tilde{M} \rightarrow M$, we have

$$
\xi^{*} \tilde{\phi}_{t}=\rho(\xi) \cdot \tilde{\phi}_{t}
$$

For each fixed $t \in[0,1]$ we define the $\rho$-twisted map

$$
\begin{aligned}
\Phi_{t} & \equiv s \circ \tilde{\phi}_{t}: \tilde{M} \rightarrow G \\
\xi^{*} \Phi_{t} & =\rho(\xi) \cdot \Phi_{t} .
\end{aligned}
$$

We study how the $\rho$-twisted map $\Phi_{t}$ varies under an infinitesimal deformation of the parameter $t$. The infinitesimal deformation is given by the vector field

$$
\delta_{t} \equiv\left(\Phi_{t}^{-1} \partial_{t} \Phi_{t}\right)_{\mathfrak{p}}
$$

which is a section of the pulled back tangent bundle

$$
\tilde{\phi}_{t}^{*} T(G / K) \rightarrow \tilde{M}
$$

equipped with the natural pulled back Levi-Civita connection on $G / K$.

We know from the proof of proposition 5 that for all $t \in[0,1]$ each of the two nonnegative terms in the integrand in the r.h.s. of (7.28) vanishes point-wise for all $t$. Using the explicit expressions for the connection and curvature on $G / K$, eqs. (7.44) and (7.45), these two conditions become

$$
d \delta_{t}+\left[\left(\Phi_{t}^{-1} d \Phi_{t}\right)_{\mathfrak{k}}, \delta_{t}\right]=\left\|\left[\left(\Phi_{t}^{-1} d \Phi_{t}\right)_{\mathfrak{p}}, \delta_{t}\right]\right\|^{2}=0,
$$

which may be combined in the single equation

$$
d \delta_{t}+\left[\Phi_{t}^{-1} d \Phi_{t}, \delta_{t}\right]=0 \quad \Rightarrow \quad d\left(\Phi_{t} \delta_{t} \Phi_{t}^{-1}\right)=0,
$$


whose solution is

$$
\Phi_{t} \delta_{t} \Phi_{t}^{-1}=v_{t} \in \mathfrak{g} \text { constant along } \tilde{M} .
$$

Consistency of eq. (7.54) with the action of the deck group (7.49) requires that the element $v_{t} \in \mathfrak{g}$ belongs to the kernel of the adjoint action of $\Gamma \equiv \rho\left(\pi_{1}(M)\right)$. By definition, this kernel is nothing else than the Lie algebra $\mathfrak{g}^{\prime \prime}$ of $G^{\prime \prime} \subset G$; then

$$
v_{t} \in \mathfrak{g}^{\prime \prime} \quad \text { for all } t \in[0,1] \text {. }
$$

Comparing eqs. (7.50) and (7.54) we get

$$
\begin{aligned}
\delta_{t} \equiv \Phi_{t}^{-1} v_{t} \Phi_{t} \in \mathfrak{p}, & \Rightarrow\left(\Phi_{t}^{-1} \partial_{t} \Phi_{t}-\Phi_{t}^{-1} v_{t} \Phi_{t}\right)_{\mathfrak{p}}=0 \\
& \Rightarrow \Phi_{t}^{-1} \partial_{t} \Phi_{t}-\Phi_{t}^{-1} v_{t} \Phi_{t} \in \mathfrak{k}
\end{aligned}
$$

Therefore by a $K$-gauge transformation (i.e. by a $t$-dependent change of trivialization $s$ of the pulled back tangent bundle) of the form

$$
\Phi_{t} \rightarrow \Phi_{t} U_{t}, \quad U_{t} \in K
$$

we may set to zero the expression inside the big parenthesis in eq. (7.56); then

$$
\left.\frac{\partial}{\partial t} \Phi_{t}=v_{t} \Phi_{t}, \quad \text { with } \quad v_{t} \in \mathfrak{g}^{\prime \prime} \text { constant along } \tilde{M} \text { (but depending on } t\right)
$$

so that the infinitesimal deformation in the geodesic family of tamed maps is produced by the action of an infinitesimal $G^{\prime \prime}$-symmetry. Integrating in $t$ we get

$$
\Phi_{1}=P \exp \left(\int_{0}^{1} v_{t} d t\right) \cdot \Phi_{0}, \quad \text { with } \quad P \exp \left(\int_{0}^{1} v_{t} d t\right) \in G^{\prime \prime},
$$

which is equivalent to the lemma.

Structure and rigidity of tamed maps. The two main results we are after are now easy consequences of the corollary.

Structural factorization. $M$ compact. Let $G^{\prime} \equiv{\overline{\rho\left(\pi_{1}(M)\right)}}^{\mathbb{R}} \subset G$ be the $\mathbb{R}$-Zariski closure of the monodromy group which we assume to be either trivial or semi-simple. Let $G^{\prime \prime} \subset G$ be the centralizer of $G^{\prime}$ in $G, K^{\prime} \subset G^{\prime}, K^{\prime \prime} \subset G^{\prime \prime}$ maximal compact subgroups, and $K \subset G$ a maximal compact subgroup containing $K^{\prime} \times K^{\prime \prime}$. Then the harmonic (in fact tamed) map $\phi: M \rightarrow \Lambda \backslash G / K$ (resp. the covering $\rho$-twisted tamed map $\tilde{\phi}$ ) factors as

$$
\begin{aligned}
& \phi: \quad M \stackrel{\phi^{\prime} \times \phi^{\prime \prime}}{\longrightarrow} \Gamma \backslash G^{\prime} / K^{\prime} \times G^{\prime \prime} / K^{\prime \prime} \stackrel{\iota 1}{\longrightarrow} \Gamma \backslash G / K, \\
\text { resp. } & \tilde{\phi}: \quad \widetilde{M} \stackrel{\tilde{\phi}^{\prime} \times \phi^{\prime \prime}}{\longrightarrow} G^{\prime} / K^{\prime} \times G^{\prime \prime} / K^{\prime \prime} \stackrel{\tilde{\iota}_{1}}{\longrightarrow} G / K,
\end{aligned}
$$

where the $\phi^{\prime \prime}: M \rightarrow G^{\prime \prime} / K^{\prime \prime}$ is a constant map. 
Were it not for the assumption that $M$ is compact, the above statement would be identical in form to the structure theorem for the period map $p$ in Hodge theory [58-61] which, in general, factorizes as in the commutative diagram

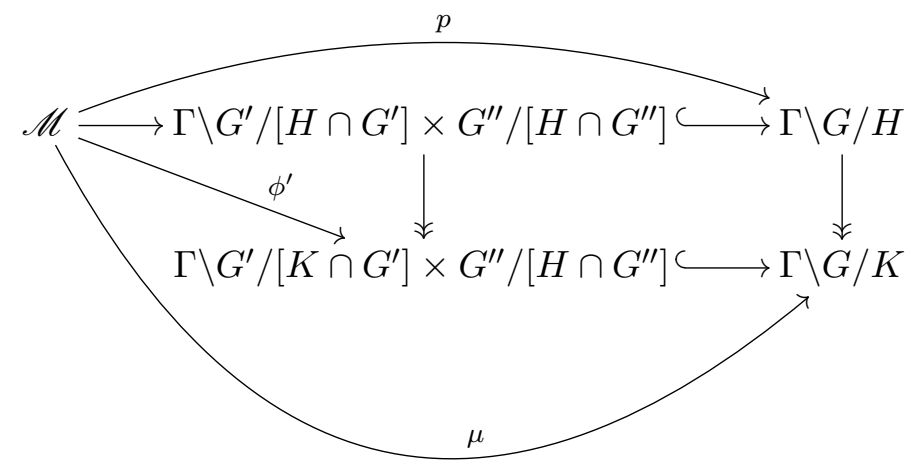

where again the maps into the factor space $G^{\prime \prime} /\left[H \cap G^{\prime \prime}\right]$ are constant. Here for a Hodge structure of odd weight $k$ and Hodge numbers $\left\{h^{p, q}\right\}[56,58-60]$

$$
H=\prod_{\substack{p+q=k \\ p<k / 2}} \mathrm{U}\left(h^{p, q}\right) \subset \mathrm{U}(h) \equiv K, \quad \text { and } \quad G=\mathrm{Sp}(2 h, \mathbb{R}), \quad \text { with } \quad 2 h=\sum_{p+q=k} h^{p, q} .
$$

The Hodge-theoretic period map $p$ satisfies, in addition to structural factorization, the infinitesimal period relations $(5.52)[56,58-60]$ — these relations are akin to the restrictions on the gauge coupling $\mu$ coming from supersymmetry: e.g. the $k=3$ Hodge structure of Calabi-Yau 3-folds are equivalent to the relations of special Kähler geometry in the sense of $\mathcal{N}=2$ SUGRA $[7,8,15]$. Most of these relations follow from the condition that the gauge coupling map is tame (e.g. if $M$ is Kähler the map is pluriharmonic and the domestic geometry reduces to $t t^{*}$ ), hence they are automatically satisfied in the present set-up.

Rigidity property. Under the assumptions above, the tamed map $\phi^{\prime}: M \rightarrow \Gamma \backslash G^{\prime} / K^{\prime}$ is rigid, that is, unique in its homotopy class.

\subsubsection{The case of OV manifolds}

In the previous subsection we got all the desired properties of domestic geometry in case $M$ is compact, which unfortunately is not a natural property in our physical applications. Our next goal is to show that in the arguments of section 7.3.1 we may drop the assumption that $M$ is compact and replace it by the two physically natural hypotheses:

(i) the source space is an OV manifold $\mathscr{M}$;

(ii) there exists a $\rho$-twisted smooth map $\tilde{\phi}_{0}$ which, when seen as a map $\phi_{0}: \mathscr{M} \rightarrow \Gamma \backslash G / K$, has finite energy $E\left(\phi_{0}\right)<\infty$.

The fact that all good properties remain true shows that of OV manifolds are really "magic". The rough idea is that a finite-energy harmonic map $\mu$ whose source is an OV space $\mathscr{M}$ behaves similarly to a map $\phi$ with a compact source space because the map $\mu$ must be "trivial at infinity" in $\mathscr{M}$. Our task is to make this idea precise. We consider the various aspects (existence, tameness, uniqueness, structural factorization, and rigidity) one by one. 
Existence. In this paragraph $M$ is any complete Riemannian manifold, compact or otherwise. Under the hypothesis (ii), it makes perfect sense to talk of continuous deformations of $\phi_{0}$ which decrease its energy, so the variational strategy for the existence/regularity problem is still meaningful: we may think of deforming continuously the map until we reach the absolute minimum value of the energy in the homotopy class defined by the monodromy representation $\rho$. The tension-flow is an efficient way of implementing the deformation in the direction of steepest descent of energy, cfr. eq. (7.1). Pursuing this strategy one gets: ${ }^{65}$

Theorem (Corlette [69]). Suppose $\rho: \pi_{1}(M) \rightarrow G$ is a homomorphism with Zariski dense image and there exists a $\rho$-twisted map $\mu$ from $M$ to $G / K$ with finite energy. Then there is a $\rho$-twisted harmonic map with finite energy from $M$ to $G / K$.

Remark 6. More generally, we may consider the case where the image $\Gamma \subset G$ of $\rho$ is Zariski dense in a non-compact, semi-simple subgroup $G^{\prime} \subset G$ (as in section 7.3.1 with "swampy" $\Gamma)$. Then the above theorem shows the existence of a finite-energy, $\rho$-twisted harmonic map $\mu$ which factorizes through a $\rho$-twisted harmonic map $\mu^{\prime}$ as in the commutative diagram

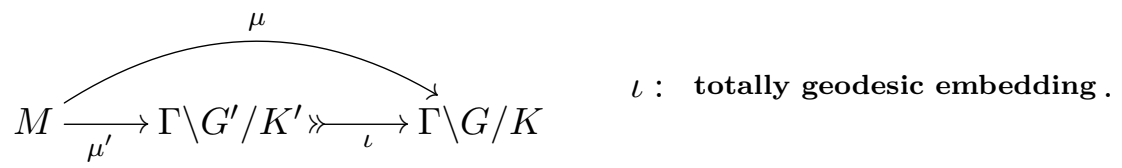

We stress that when $G^{\prime} \subsetneq G$ the above theorem, by itself, says nothing about the possible existence of non-factorized harmonic maps, i.e. it refers only to the existence of harmonic maps enjoying the structural factorization (7.63). Below we shall see that all finite-action $\rho$-twisted harmonic maps are factorized as in (7.63).

Roughly speaking, finite energy corresponds to finite volume of the image $\mu(\mathscr{M})$ : thus if the scalars' manifold $\mathscr{M}$ satisfies (our slightly stronger version of) the standard swampland conjectures, any gauge-coupling map $\mu: \mathscr{M} \rightarrow \Gamma \backslash \operatorname{Sp}(2 h, \mathbb{Z}) / \mathrm{U}(g)$ is homotopic to a harmonic one, namely the fixed point $\mu_{\text {harm }}$ of the tension flow with initial condition $\mu$. The heuristic physical arguments of section 7.2 suggest that, in the extreme IR limit of a consistent quantum gravity, the physical coupling $\mu_{\text {phys }}$ actually coincides with $\mu_{\text {harm }}$ at least approximately and away from finite-distance singularities. We stress that this statement is literally true in all known examples of reliable quantum-consistent effective theories of gravity.

Tameness. The Bochner argument around eq. (7.23) still works in the non-compact case provided we can show that the surface term in the integration over $\mathscr{M}$ of the l.h.s. of (7.23) vanishes; in this case we conclude that the finite-action $\rho$-twisted harmonic map $\mu$ is actually tamed. Thus to show the tamed property for OV manifolds stated at the beginning of section 7.1, we have only to justify the dropping of the boundary term in the integration by parts of the l.h.s. in eq. (7.23) under our two assumptions that $\mathscr{M}$ is OV and $\mu$ has finite energy. We defer this technicality to appendix B.

\footnotetext{
${ }^{65}$ Warning. In section 7.3.2 we adopt the terminology of Corlette [69]: a $\rho$-equivariant map $f: \tilde{M} \rightarrow G / K$ is called a $\rho$-twisted map $M \rightarrow G / K$ (instead of a $\rho$-twisted map $\tilde{M} \rightarrow G / K$ as it is more natural in the physical parlance). A $\rho$-twisted $\operatorname{map} M \rightarrow G / K$ can also be defined as a section of the bundle $\tilde{M} \times{ }_{\rho} G / K[69]$.
} 
Essential uniqueness, structural factorization, and rigidity. As explained in the footnote 62, the crucial equation (7.26) holds for any geodesic family of finite-energy maps whether the manifold $\mathscr{M}$ is compact or not. The only way compactness enters in the game is that it guarantees the finite-energy condition for all smooth maps, whereas in the noncompact case one should add the finite-energy condition as an independent hypothesis and hence the results apply only to a small sub-class of harmonic maps. Therefore, when the finite-energy condition is satisfied, all formal consequences of (7.26) follow. In particular all finite-energy tamed maps, if non-rigid, belong to one-parameter families of maps with the same energy. In turn this leads to essential uniqueness of the finite-energy tamed maps (in their homotopy class) in the sense of definition 7. In particular, the argument at the end of section 7.3.1 yield

Structural factorization. All finite-energy harmonic (hence tamed) $\rho$-twisted maps

$$
\mu: \mathscr{M} \rightarrow \Gamma \backslash G / K
$$

factorize as in eq. (7.63). They form a $G^{\prime \prime} / K^{\prime \prime}$-family on which the $\sigma$-model symmetry group $G^{\prime \prime}$ acts transitively. (Concretely $G^{\prime \prime}$ acts by varying the geodesic embedding $\iota$ in (7.63).)

The same argument shows that the factor map $\mu^{\prime}$ in (7.63) is rigid.

Nothing is said about the harmonic/tamed maps of infinite energy (which are expected to be the large majority).

\subsubsection{Structure of the gauge coupling $\mu$}

We have seen above that in (arithmetic) domestic geometry the crucial structural factorization holds for a gauge coupling $\mu$. Here we write this property in a more detailed (and convenient) form. To simplify the formulae, we omit writing the boring factor space $G^{\prime \prime} / K^{\prime \prime}$ and the trivial constant map into it. ${ }^{66}$ With this convention, $\mu$ factorizes as in the commutative diagram

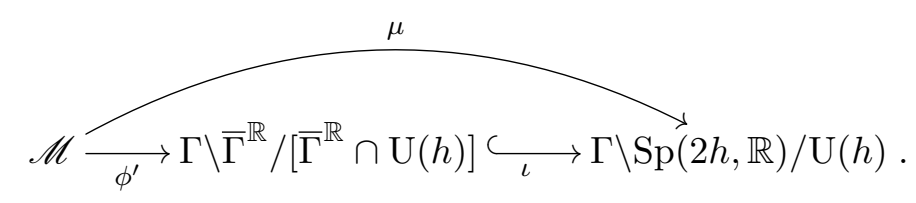

If $\mu$ is harmonic, the real Lie group (or, rather, algebraic group over $\mathbb{R}$ ) $\bar{\Gamma}^{\mathbb{R}} \subset \operatorname{Sp}(2 h, \mathbb{R}$ ) must be reductive. Hence, modulo finite groups, it has the form

$$
\bar{\Gamma}^{\mathbb{R}} \cong A \times G_{1} \times \cdots \times G_{s}
$$

with $A$ Abelian and $G_{\ell}$ simple. Correspondingly (up to commensurability [22])

$$
\Gamma \cong \Gamma_{A} \times \Gamma_{1} \times \cdots \times \Gamma_{s}
$$

\footnotetext{
${ }^{66}$ For instance, in $\mathcal{N}=2$ supergravity we write the gauge coupling as a function of the vector-multiplet scalars, instead of a function of all scalars which is constant in the hypermultiplet scalars.
} 
with $\Gamma_{A} \subset A$ and $\Gamma_{\ell} \subset G_{\ell}$. Since we are assuming $\Gamma$ to be neat and generated by unipotents, $\Gamma_{A}$ must be trivial. Then the real Lie group $\bar{\Gamma}^{\mathbb{R}} \subset \operatorname{Sp}(2 h, \mathbb{R})$ is either trivial or semisimple. In the fist case the gauge couplings $\mu$ are field-independent numerical constants. An example of this situation is given by the compactification of Type IIB on a rigid CalabiYau [51].

If $\Gamma$ is not trivial, the harmonic map $\phi^{\prime}$ decomposes into a $s$-tuple of partial maps

$$
\phi_{\ell}: \mathcal{M} \rightarrow \Gamma_{\ell} \backslash G_{\ell} /\left[G_{\ell} \cap \mathrm{U}(h)\right], \quad \ell=1, \cdots, s .
$$

We stress that all spaces through which the gauge coupling $\mu$ factorizes, i.e. $\Gamma \backslash \bar{\Gamma}^{\mathbb{R}} /\left[K \cap \bar{\Gamma}^{\mathbb{R}}\right]$ and the $\Gamma_{\ell} \backslash G_{\ell} /\left[G_{\ell} \cap K\right]$ are OV manifolds.

As already mentioned, the "structural factorization" (7.65) is identical in form ${ }^{67}$ to the structure theorem for Griffiths period maps in modern Hodge theory [58-61], which is satisfied by the low-energy effective theories of Type II compactified on a geometric family of Calabi-Yau, whose couplings are determined by the Griffiths period map [7,8], and which is the main condition discriminating the quantum consistent $\mathcal{N}=2$ supergravities from the ones belonging to the swampland [15]. In particular, whenever the $\mathcal{N}=2$ SUGRA satisfies the structure theorem automatically satisfies all the relevant swampland conjectures, see $[15]$.

It is remarkable that the very same structural properties hold in full generality — even when the moduli space $\mathscr{M}$ has general holonomy $\mathfrak{s o}(m)$ and no natural complex structure - by virtue of the "magical" properties of the OV manifolds, provided we add to the list of the swampland conjectures the statement that the IR gauge coupling $\mu$ is harmonic of finite-energy.

We take the above state of affairs as evidence that our working hypothesis is somehow on the right track.

\subsubsection{Swampland conditions for $\mathcal{N}=2$ SUGRA}

This paragraph is a comment about reference [15]. As we know, on SUSY grounds, the vector-multiplet couplings of a $4 \mathrm{~d} \mathcal{N}=2$ SUGRA are dictated by special Kähler geometry which is equivalent to a variation of Hodge structure of weight 3 with $h^{3,0}=1$. In [15] it was observed that a deep problem in math is to determine which VHS arise from geometry, i.e. describes an actual family of Calabi-Yau manifolds. A condition mathematicians have proven to be necessary is that the period map $p$ of the VHS satisfies the structure theorem [58-61]. It was proposed in [15] that this same condition is also a swampland criterion for $4 \mathrm{~d} \mathcal{N}=2$ effective theories. Then the question was the logic relation between this new criterion and the Ooguri-Vafa geometric swampland conjectures [2]. The fact that the validity of the structure theorem of [61] implies the swampland conjecture is easy to see [15]. It was initially believed that the structure theorem was a stronger requirement than the Ooguri-Vafa ones: the naive feeling was that the structure theorem is a stringent condition with lots of Number Theoretical and Algebro-Geometric aspects whereas the

\footnotetext{
${ }^{67}$ The Griffiths period map satisfies in addition the IPR, so we have the more detailed factorization of $\mu$ in the diagram (7.61).
} 
OV statements looked like simple qualitative properties of the relevant geometries. However, now we see that the OV properties are strong enough (when supplemented by the conditions following from $\mathcal{N}=2$ supersymmetry and some mild regularity assumption) to actually imply the structure theorem of [61], so that the two set of conditions are essentially equivalent.

\subsection{First applications}

In the context of $4 \mathrm{~d} \mathcal{N}=2$ supergravity, the Hodge-theoretic structure theorem - which holds only in a tiny subset of the space of all formal $\mathcal{N}=2$ sUGRA which includes all the ones arising from string theory - has a lot of interesting implications [15], which include the completeness of instanton corrections [98].

If our working hypothesis is correct, a structure theorem of exactly the same form holds for all consistent effective theories of quantum gravity. However the powerful results in the $\mathcal{N}=2$ situation arise from the interplay between two pieces of information: the structure theorem and special Kähler geometry. In the non-SUSY case, where $\mathscr{M}$ has generic holonomy, the second ingredient is lacking, and we are able to extract from the structure theorem much weaker physical consequences - which however have the merit of being (conjecturally) true in full generality.

The $\mathcal{N}=2$ case. Let us briefly recall the situation in the $\mathcal{N}=2$ context. ${ }^{68}$ In this case the gauge coupling $\mu$, seen as a fibration over its image $\mathscr{B}$,

$$
\mu: \mathscr{M} \rightarrow \mathscr{B} \equiv \mu(\mathscr{M}) \subset \Gamma \backslash \mathrm{Sp}(2 h, \mathbb{R}) / \mathrm{U}(h)
$$

is essentially trivial in the sense that $\mathscr{M}=\mathscr{M}_{\text {hyper }} \times \mathscr{M}_{\text {vector }}$, and the Griffiths' infinitesimal period relations [56, 58-60], together with the Torelli theorem [29-31], say that the period map $p$ is a Griffiths-horizontal, holomorphic embedding of the universal cover of $\mathscr{M}_{\text {vector }}$ into the Griffiths period domain $\boldsymbol{D}_{h}$ (cfr. eq. (7.62))

$$
p: \widetilde{\mathscr{M}}_{\text {vector }} \rightarrow \boldsymbol{D}_{h} \stackrel{\text { def }}{=} \operatorname{Sp}(2 h, \mathbb{R}) /[\mathrm{U}(1) \times \mathrm{U}(h-1)] .
$$

Composing with the canonical projection $\boldsymbol{D}_{h} \rightarrow \boldsymbol{H}_{h}$, we see that the non-holomorphic smooth map $\widetilde{\mathscr{M}}_{\text {vector }} \rightarrow \widetilde{\mathscr{B}} \equiv \widetilde{\mu}(\widetilde{\mathscr{M}})$ is a local isomorphism by horizontality of $\tilde{p}$. The Kähler form on $\widetilde{\mathscr{M}}_{\text {vector }}$ is pull back $\tilde{p}^{*} F$ of the curvature 2-form $F$ of the Hodge line bundle $\mathcal{L} \rightarrow \boldsymbol{D}_{h}$ (i.e. the homogeneous bundle over $\boldsymbol{D}_{h}$ defined by the fundamental character of the $\mathrm{U}(1)$ factor in $H=\mathrm{U}(1) \times \mathrm{U}(h-1))$. Then in the $\mathcal{N}=2$ case there is a simple relation between the gauge couplings $\tau(\phi)_{a b}$ and the scalar metric $G(\phi)_{i j}$ expressed by the moduli space Einstein equation (6.7). In particular, all isometries of $\widetilde{\mathscr{M}}_{\text {vector }} \hookrightarrow \boldsymbol{D}_{h}$ is the restriction of an $\operatorname{Sp}(2 h, \mathbb{R})$ symmetry of the ambient space $\boldsymbol{D}_{h}$.

The general case. In the case of a general quantum-consistent effective theory - with NO supersymmetry - we do not expect a simple relation between the couplings $\tau(\phi)_{a b}$ and $G(\phi)_{i j}$. However, to the extend that $\mu$ is harmonic, quantum consistency still implies subtle

\footnotetext{
${ }^{68}$ Our summary below is rather rough and the statements are not meant to be technically precise; see [15] for a more precise discussion.
} 
relations between the two couplings. In particular the scalars' metric $G(\phi)_{i j}$ is constrained by the condition that the gauge coupling $\mu$ is harmonic for $G(\phi)_{i j}$. This severely restricts the allowed scalar metric. E.g., when $\mathscr{M}$ is a complex OV manifold and $\mu$ is pluri-harmonic, it requires $G(\phi)_{i j}$ to be Kähler. The scalars metric satisfies also other strong constraints:

(i) the infinite group $\mathcal{G}$ acts by isometries on $G(\phi)_{i j}$, (ii) the volume is finite, and (iii) Ricci curvature satisfies the required bounds. Thus, even if the effective theory has no SUSY, for a given gauge coupling $\mu$ there is not that much freedom in the choice of the scalars' metric $G(\phi)_{i j}$ if we wish to avoid ending in the swampland.

Unfortunately, for a non-SUSY theory the relation between $\mu$ and the consistent scalar's metric $G(\phi)_{i j}$ is rather implicit. For this reason, in absence of SUSY it is hard to rephrase the structure theorem for $\mu$ in terms of geometric proprieties of $G(\phi)_{i j}$.

The structure theorem refers to properties of $\mathscr{B} \equiv \mu(\mathscr{M})$, seen as a submanifold of the Siegel variety $\operatorname{Sp}(2 h, \mathbb{Z}) \backslash \boldsymbol{H}_{h}$, rather than directly to the intrinsic geometry of $\mathscr{M}$. In the $\mathcal{N}=2$ case $\mathscr{B} \cong \mathscr{M}_{\text {vector }}$ so this is not a limitation, but in general the two spaces are quite different. Anyhow the $\mathcal{N}=2$ statements of [15] hold for general domestic geometries when referred to $\mathscr{B}$. In particular we have the dycothomy:

(a) either $\widetilde{\mathscr{B}} \subset \boldsymbol{H}_{h}$ is a totally geodesic submanifold, hence symmetric, and the fibers $\tilde{\mu}^{-1}(b) \subset \widetilde{\mathscr{M}}$ are minimal submanifolds;

(b) or no continuous symmetry of the ambient space $\boldsymbol{H}_{h}$ leaves $\widetilde{\mathscr{B}}$ fixed (as a set).

Possibility (a) corresponds to very special effective theories which look like consistent truncations of some $\mathcal{N} \geq 3$ supegravity. The second possibility is the generic case. In the $\mathcal{N}=2$ case this implies completeness of instanton corrections (which is expected on physical grounds [98]), and this implication is likely to extend to more general situations.

\section{A SUGRA spaces: tamed maps vs. special holonomy}

We want to show that if $X$ is a symmetric space relevant for $4 \mathrm{~d}$ SUGRA not of the form $\mathrm{SO}(m, 1) / \mathrm{SO}(m)$ or $\mathrm{SU}(m, 1) / \mathrm{U}(m)$ all tamed maps $f: X \rightarrow Y$ are totally geodesic.

We consider the symmetric Riemannian manifolds of type III, i.e. of the form $G / K$ where $G$ is some real Lie algebra and $K$ its maximal compact subgroup. By general theory its holonomy Lie algebra is $\mathfrak{k} \equiv \mathfrak{L} \mathfrak{i}(K)$. The space $\mathrm{SO}(m, 1) / \mathrm{SO}(m)$ has dimension $m$ and strictly generic holonomy algebra $\mathfrak{s o}(m)$, so has no non-trivial parallel forms, and hence in this case tamed $\equiv$ harmonic. The complex hyperbolic space $\mathrm{SU}(m, 1) / \mathrm{U}(m)$ has complex dimension $m$ and holonomy Lie algebra $\mathfrak{s u}(m)$, so it is a strict Kähler manifold and hence for the complex hyperbolic spaces tamed $\equiv$ pluri-harmonic. We shall call $\mathrm{SO}(m, 1) / \mathrm{SO}(m)$ and $\mathrm{SU}(m, 1) / \mathrm{U}(m)$ the strict cases. For all other type III symmetric spaces the holonomy algebra $\mathfrak{h o l}(G / K)$ is neither generic nor strict Kähler, so for these spaces tamed is strictly stronger than being harmonic or pluri-harmonic. We consider the cosets $G / K$ relevant for 4d SUGRA.

We write $T \cong T^{\vee}$ for the (irreducible) holonomy representation of the symmetric space $G / K$. The corresponding Lie algebras decomposes as $\mathfrak{g}=\mathfrak{k} \oplus T$ and the holonomy 
representation on $T$ is induced by the adjoint action of $\mathfrak{g}$ on itself. $G / K$ has a non-trivial algebra $\mathcal{P}^{\bullet}$ of parallel forms $\Omega^{(s)} \in \wedge^{k_{s}} T$. We consider their annihilator algebra

$$
\mathfrak{a} \stackrel{\text { def }}{=}\left\{a_{i j} \in \otimes^{2} T \cong \operatorname{End}(T): a_{\left[i_{1 j} j\right.} \Omega_{\left.j i_{2} \cdots i_{k_{s}}\right]}^{(s)}=0 \quad \forall \Omega^{(s)} \in \mathcal{P}^{\bullet}\right\} .
$$

A map $f$ is tamed iff $D_{i} \partial_{j} f$ is contained in $\mathfrak{a} \cap \odot^{2} T$; when this space is zero and $f$ is tamed we must have $D_{i} \partial_{j} f=0$, that is,

$$
\mathfrak{a} \cap \odot^{2} T=0 \quad \Longrightarrow \quad \text { all tamed maps are totally geodesic. }
$$

$\mathfrak{a} \subset \otimes^{2} T$, is a real Lie subalgebra of $\mathfrak{s l}(T)$, contains $\mathfrak{k}$ and is a $\mathfrak{k}$-invariant subspace; hence it has the form $\mathfrak{a}=\mathfrak{k} \oplus \mathfrak{b}$ with $\mathfrak{b} \subset\left(\otimes^{2} T\right)_{\text {traceless. }}$. The algorithm goes through the following steps. (1) we show that $\mathfrak{a} \cap \wedge^{2} T=\mathfrak{k}$ while all irreducible $\mathfrak{k}$-representations in $\left(\odot^{2} T\right)_{\text {traceless }}$ are self-dual, so we infer that $\mathfrak{a}$ is a reductive Lie algebra with maximal compact subalgebra $\mathfrak{k}$. Writing $A, K$ for the corresponding group, $A / K$ is a, possibly trivial or reducible, symmetric space. (2) One checks in the Cartan table of symmetric space which groups $A, K$ are allowed and reads from them the candidate $\mathfrak{b}$. (3) Finally one checks that the candidate $\mathfrak{b} \not \subset \odot{ }^{2} T$, getting a paradox. (4) We conclude that $\mathfrak{a} \cap \odot^{2} T=0$ and apply (A.2).

We run the algorithm one space at the time.

- $\mathcal{N}=8$ sugra. The scalars' space is $E_{7(7)} / \mathrm{SU}(8) . K=\mathrm{SU}(8)$ and $T$ is the $\mathbf{7 0}$ i.e. $T=\wedge^{4} F(F$ stands for the fundamental of $\mathrm{SU}(8)) . \wedge{ }^{6} T$ contains a singlet, i.e. on the symmetric space

$$
E_{7(7)} / \mathrm{SU}(8)
$$

we have a non-trivial parallel 6 -form and $\mathfrak{s o}(T) \not \subset \mathfrak{a}$. On the other hand, as $\mathfrak{s u}(8)$ modules

$$
\mathfrak{s o}(T)=\wedge^{2}\left(\wedge^{4} F\right)=\mathfrak{s u}(8) \oplus \mathbf{2 3 5 2}
$$

so $\mathfrak{s u}(8)$ is the maximal compact subalgebra of $\mathfrak{a}$. On the other hand

$$
\odot^{2} T=\odot^{2}\left(\wedge^{4} F\right)=\mathbf{7 2 0} \oplus \mathbf{1 7 6 4}
$$

and both representations are self-dual. Hence $\mathfrak{a}$ is semi-simple with maximal compact subalgebra $\mathfrak{s u}(8)$ and $A / K$ is a non-compact symmetric space. Since $E_{7(7)} / \mathrm{SU}(8)$ is the only non-trivial non-compact symmetric space of holonomy $\mathrm{SU}(8)$, we must have either $\mathfrak{a} \cap \odot^{2} T$ equal zero or $T$. But $T \not \subset \odot^{2} T$ and the second possibility is ruled out.

- $\mathcal{N}=6$ SUgra. The scalars' space $S O^{*}(12) / \mathrm{U}(6)$ is Kähler; $T=\left(\wedge^{2} F \oplus \wedge^{2} \bar{F}\right)_{\mathbb{R}}(F$ is the fundamental of $\mathrm{U}(6))$. Then

$$
\wedge^{2} T=\left(\wedge^{2} F\right) \otimes\left(\wedge^{2} \bar{F}\right) \oplus\left(\wedge^{2}\left(\wedge^{2} F\right) \oplus \wedge^{2}\left(\wedge^{2} \bar{F}\right)\right)_{\mathbb{R}}=\mathbf{1} \oplus \mathfrak{s u}(6) \oplus \mathbf{1 8 9} \oplus(\mathbf{1 0 5} \oplus \overline{\mathbf{1 0 5}})_{\mathbb{R}}
$$

where 1 is the Kähler form. $\wedge^{3}\left(\wedge^{2} F\right) \otimes \wedge^{3}\left(\wedge^{2} \bar{F}\right)$ contains 2 singlets, so that we have a parallel $(3,3)$ form different from the cube of the Kähler form and $\mathfrak{u}(15) \not \subset \mathfrak{a}$. On the other and

$$
\mathfrak{u}(15)=\left(\wedge^{2} F\right) \otimes\left(\wedge^{2} \bar{F}\right)=\mathfrak{u}(6) \oplus \mathbf{1 8 9}
$$


so the maximal compact subalgebra of $\mathfrak{a}$ is $\mathfrak{u}(6)$. Now we have 3 non-trivial symmetric spaces to consider namely $\mathrm{SU}(6,1) / \mathrm{U}(6), \mathrm{Sp}(12, \mathbb{R}) / \mathrm{U}(6)$ and $S O^{*}(12) / \mathrm{U}(6)$ with would-be $\mathfrak{b},(F \oplus \bar{F})_{\mathbb{R}},\left(\odot^{2} F \oplus \odot^{2} \bar{F}\right)_{\mathbb{R}}$ and $\left(\wedge^{2} F \oplus \wedge^{2} \bar{F}\right)_{\mathbb{R}}$, respectively. Since $\odot^{2} T$ contains only the $\mathrm{U}(1)$ characters $4,0,-4$ we get a contradiction in all cases. We conclude that $\mathfrak{b}=0$, so $\mathfrak{a} \cap \odot^{2} T=0$.

- $\mathcal{N}=5$ sugra has $G / K=\mathrm{SU}(5,1) / \mathrm{U}(5)$ which is a strict case.

- $\mathcal{N}=4$ SugRa. The (universal cover of) the scalars' space is reducible

$$
\mathrm{SU}(1,1) / \mathrm{U}(1) \times \mathrm{SO}(6, k) /[\mathrm{SO}(6) \times \mathrm{SO}(k)],
$$

the first factor is strict, as it is the second one when $k=1$. We focus on the second factor and assume $k \geq 2$ then $T=V_{6} \otimes V_{k}$, where $V_{k}$ is the vector of $\operatorname{SO}(k)$. We have a parallel 6 -form and dually a parallel $6(k-1)$ form, hence $\mathfrak{s o}(6 k) \not \subset \mathfrak{a}$. Since

$$
\wedge^{2} T=\mathfrak{s o}(6) \otimes\left(\mathbf{1} \oplus\left(\odot^{2} V_{k}\right)_{\text {traceless }}\right) \oplus(\mathbf{1} \oplus \mathbf{2 0}) \otimes \mathfrak{s o}(k)
$$

we have that $\mathfrak{s o}(6) \oplus \mathfrak{s o}(k)$ is the maximal compact subalgebra of $\mathfrak{a}$. We have two possible non-trivial symmetric spaces

$$
\mathrm{SL}(6, \mathbb{R}) / \mathrm{SO}(6) \times \mathrm{SL}(k, \mathbb{R}) / \mathrm{SO}(k) \quad \text { and } \quad \mathrm{SO}(6, k) /[\mathrm{SO}(6) \times \mathrm{SO}(k)]
$$

with would-be $\mathfrak{b} \subset\left(\odot^{2} V_{6}\right)_{\text {traceless }} \oplus\left(\odot^{2} V_{k}\right)_{\text {traceless }}$ and $\mathfrak{b} \subset V_{6} \otimes V_{k}$, respectively. The first one obviously does not preserve the parallel forms, and the second one is not contained in $\odot^{2} T$. This rules out also $\mathrm{SL}(6, \mathbb{R}) / \mathrm{SO}(6)$ and $\mathrm{SL}(k, \mathbb{R}) / \mathrm{SO}(k)$ and one remains with $\mathfrak{a}=\mathfrak{s o}(6) \oplus \mathfrak{s o}(k)$.

- $\mathcal{N}=3$ SUgra. $\mathrm{SU}(3, k) /[\mathrm{SU}(3) \times \mathrm{U}(k)]$ again is Kähler and for $k=1$ strict. $T=$ $\left(F_{3} \otimes F_{k} \oplus \bar{F}_{3} \otimes \bar{F}_{k}\right)_{\mathbb{R}}$. We have parallel $(3,3)$ and $(k, k)$ forms, the maximal compact subalgebra is $\mathfrak{s u}(3) \oplus \mathfrak{u}(k)$; going through the various symmetric spaces, one concludes that $\mathfrak{b}=0$.

\section{B No boundary term in the Bochner argument}

As explained at the end of section 7.3 , we have to show that if $\mathscr{M}$ is a OV manifold and $f: \mathscr{M} \rightarrow \Lambda \backslash G / K$ is a finite-energy harmonic map to a locally symmetric space of non-compact type, the surface term

$$
\int_{\mathscr{M}} d\left(g_{i j} *\left(d f^{i} \wedge \Omega\right) \wedge D *\left(d f^{j} \wedge * \Omega\right)\right)
$$

vanishes (cfr. eq. (7.23)). We proceed by adapting the argument in [69]. As in section 2.4.2, for all $R>0$ we write $h_{R}: \mathscr{M} \rightarrow \mathbb{R}$ for a Lipschitz continuous function such that for some fixed constant $k>0$ [37]:

$$
0 \leq h_{R} \leq 1, \quad h_{R}=\left\{\begin{array}{ll}
1 & \text { for } r \leq R \\
0 & \text { for } r \geq 2 R,
\end{array} \quad\left|d h_{R}\right|<\frac{k}{R},\right.
$$


and assume condition (*) i.e. eq. (2.24)

$$
\left|\Delta h_{R}\right|<C .
$$

We then proceed as in reference [69]:

$$
\left.\left.\left|\int_{\mathscr{M}} h_{R} \Delta\right| d f\right|^{2} d \mathrm{vol}|=| \int_{\mathscr{M}}\left(\Delta h_{R}\right)|d f|^{2} d \mathrm{vol}\left|\leq \int_{\mathscr{M}}\right| \Delta h_{R}|| d f\right|^{2} d \mathrm{vol} \leq C E(f)
$$

and taking $R \rightarrow \infty$

$$
\left.\left|\int_{\mathscr{M}} \Delta\right| d f\right|^{2} d \mathrm{vol} \mid \leq C E(f)
$$

Now let $f: \mathscr{M} \rightarrow \Lambda \backslash G / K$ be harmonic of finite energy, $E(f)<\infty$. Since the target space has non-positive sectional curvatures, and the Ricci tensor of $\mathscr{M}$ is bounded below (cfr. eq. (3.4)),

$$
R_{i j} \geq-K g_{i j}
$$

the Bochner formula of Eells and Sampson for harmonic maps [87 ${ }^{69}$

$$
\frac{1}{2} \Delta|d f|^{2}=|\nabla d f|^{2}+R_{i j} h_{a b} \partial^{i} f^{a} \partial^{j} f^{b}-R_{a b c d}^{h} g^{i k} g^{j l} \partial_{i} f^{a} \partial_{j} f^{b} \partial_{k} f^{c} \partial_{l} f^{d}
$$

gives a bound of the form

$$
\left|D_{i} \partial_{j} f\right|^{2} \leq \frac{1}{2} \Delta|d f|^{2}+K|d f|^{2} \Rightarrow \int_{\mathscr{M}}\left|D_{i} \partial_{j} f\right|^{2} d \mathrm{vol} \leq\left(\frac{1}{2} C+K\right) E(f) .
$$

This bound implies that both $\left(d f \wedge \Omega^{(s)}\right)$ and $D *\left(d f \wedge * \Omega^{(s)}\right)$ have finite $L^{2}$ norms. Now the boundary term that we have to show to vanish, (B.1), is the limit as $R \rightarrow \infty$ of

$$
\int_{\mathscr{M}} h_{R} d\langle *(d f \wedge \Omega), D *(d f \wedge * \Omega)\rangle=-\int_{\mathscr{M}} d h_{R} \wedge\langle *(d f \wedge \Omega), D *(d f \wedge * \Omega)\rangle
$$

while $\left|d h_{R}\right| \leq k / R$ so that

$$
\left|\int_{\mathscr{M}} d h_{R} \wedge\langle *(d f \wedge \Omega), D *(d f \wedge * \Omega)\rangle\right| \leq \frac{k}{R}\|d f \wedge \Omega\|_{L^{2}} \cdot\|D *(d f \wedge * \Omega)\|_{L^{2}}
$$

which goes to zero as $R \rightarrow \infty$.

Open Access. This article is distributed under the terms of the Creative Commons Attribution License (CC-BY 4.0), which permits any use, distribution and reproduction in any medium, provided the original author(s) and source are credited.

\section{References}

[1] C. Vafa, The String landscape and the swampland, hep-th/0509212 [INSPIRE].

[2] H. Ooguri and C. Vafa, On the Geometry of the String Landscape and the Swampland, Nucl. Phys. B 766 (2007) 21 [hep-th/0605264] [INSPIRE].

\footnotetext{
${ }^{69} h_{a b}$ and $R_{a b c d}^{h}$ are, respectively, the metric and the Riemann tensor of the target space.
} 
[3] T.D. Brennan, F. Carta and C. Vafa, The String Landscape, the Swampland, and the Missing Corner, PoS TASI2017 (2017) 015 [arXiv: 1711.00864] [INSPIRE].

[4] E. Palti, The Swampland: Introduction and Review, Fortsch. Phys. 67 (2019) 1900037 [arXiv: 1903.06239] [INSPIRE].

[5] T. Banks and N. Seiberg, Symmetries and Strings in Field Theory and Gravity, Phys. Rev. D 83 (2011) 084019 [arXiv: 1011.5120] [INSPIRE].

[6] H.-C. Kim, H.-C. Tarazi and C. Vafa, Four-dimensional $\mathcal{N}=4 S Y M$ theory and the swampland, Phys. Rev. D 102 (2020) 026003 [arXiv:1912.06144] [InSPIRE].

[7] S. Cecotti, $N=2$ Supergravity, Type IIB Superstrings and Algebraic Geometry, Commun. Math. Phys. 131 (1990) 517 [inSPIRE].

[8] A. Strominger, Special geometry, Commun. Math. Phys. 133 (1990) 163 [InSPIRE].

[9] T.W. Grimm, E. Palti and I. Valenzuela, Infinite Distances in Field Space and Massless Towers of States, JHEP 08 (2018) 143 [arXiv: 1802.08264] [INSPIRE].

[10] T.W. Grimm, C. Li and E. Palti, Infinite Distance Networks in Field Space and Charge Orbits, JHEP 03 (2019) 016 [arXiv:1811.02571] [INSPIRE].

[11] P. Corvilain, T.W. Grimm and I. Valenzuela, The Swampland Distance Conjecture for Kähler moduli, JHEP 08 (2019) 075 [arXiv: 1812.07548] [INSPIRE].

[12] A. Joshi and A. Klemm, Swampland Distance Conjecture for One-Parameter Calabi-Yau Threefolds, JHEP 08 (2019) 086 [arXiv: 1903.00596] [INSPIRE].

[13] T.W. Grimm and D. van de Heisteeg, Infinite Distances and the Axion Weak Gravity Conjecture, JHEP 03 (2020) 020 [arXiv:1905.00901] [INSPIRE].

[14] T.W. Grimm, F. Ruehle and D. van de Heisteeg, Classifying Calabi-Yau Threefolds Using Infinite Distance Limits, Commun. Math. Phys. 382 (2021) 239 [arXiv:1910.02963] [INSPIRE].

[15] S. Cecotti, Special Geometry and the Swampland, JHEP 09 (2020) 147 [arXiv:2004.06929] [INSPIRE].

[16] C. Lazaroiu and C.S. Shahbazi, The duality covariant geometry and DSZ quantization of Abelian gauge theory, arXiv:2101.07236 [INSPIRE].

[17] C.I. Lazaroiu and C.S. Shahbazi, The geometry and DSZ quantization of four-dimensional supergravity, arXiv:2101.07778 [INSPIRE].

[18] T.W. Grimm, Moduli Space Holography and the Finiteness of Flux Vacua, arXiv:2010.15838 [INSPIRE].

[19] B. Bastian, T.W. Grimm and D. van de Heisteeg, Weak gravity bounds in asymptotic string compactifications, JHEP 06 (2021) 162 [arXiv:2011.08854] [INSPIRE].

[20] H. Ooguri and C. Vafa, Non-supersymmetric AdS and the Swampland, Adv. Theor. Math. Phys. 21 (2017) 1787 [arXiv: 1610.01533] [InSPIRE].

[21] N. Seiberg, The Power of holomorphy: Exact results in $4 D$ SUSY field theories, in proceedings of the Particles, Strings, and Cosmology (PASCOS 94), Syracuse, NY, U.S.A., 19-24 May 1994, pp. 357-369 [hep-th/9408013] [INSPIRE].

[22] D.W. Morris, Introduction to Arithmetic Groups, math.DG/0106063. 
[23] C.-L. Wang, On the incompleteness of the Weil-Petersson metric along degenerations of Calabi-Yau manifolds, Math. Res. Lett. 4 (1997) 157.

[24] M. Reid, The moduli space of 3-folds with $K=0$ may nevertheless be irreducible, Math. Ann. 278 (1987) 329.

[25] S. Cecotti and C. Vafa, Ising model and $N=2$ supersymmetric theories, Commun. Math. Phys. 157 (1993) 139 [hep-th/9209085] [INSPIRE].

[26] M. Bershadsky, S. Cecotti, H. Ooguri and C. Vafa, Holomorphic anomalies in topological field theories, in AMS/IP Studies in Advanced Mathematics 1, American Mathematical Society, Providence RI U.S.A. (1996), pp. 655-682 [Nucl. Phys. B 405 (1993) 279] [hep-th/9302103] [INSPIRE].

[27] Z. Lu, On the Hodge metric of the universal deformation space of Calabi-Yau threefolds, math.DG/0505582.

[28] S. Cecotti, Moduli spaces of Calabi-Yau d-folds as gravitational-chiral instantons, JHEP 12 (2020) 008 [arXiv: 2007.09992] [INSPIRE].

[29] X. Chen, K. Liu and Y. Shen, Global Torelli theorem for projective manifolds of Calabi-Yau type, arXiv:1205.4207v3.

[30] K. Liu and Y. Shen, Hodge metric completion of the moduli space of Calabi-Yau manifolds, arXiv: 1305.0231.

[31] K. Liu and Y. Shen, From local Torelli to global Torelli, arXiv:1512.08384.

[32] A.L. Besse, Einstein manifolds, in Ergebnisse der Mathematik und ihrer Grenzgebiete, Springer (1987).

[33] J.W. Morgan and G. Tian, Ricci Flow and the Poincaré Conjecture, math.DG/0607607.

[34] A. Kehagias, D. Lüst and S. Lüst, Swampland, Gradient Flow and Infinite Distance, JHEP 04 (2020) 170 [arXiv: 1910.00453] [INSPIRE].

[35] T. Trenner and P.M.H. Wilson, Asymptotic curvature of moduli spaces of Calabi-Yau threefolds, J. Geom. Anal. 21 (2011) 409 [arXiv:0902.4611].

[36] S. Cecotti, Supersymmetric Field Theories. Geometric Structures and Dualities, Cambridge University Press, Cambridge U.K. (2015) [https://doi.org/10.1017/CBO9781107284203].

[37] S.-T. Yau, Some function-theoretic properties of complete Riemannian manifold and their applications to geometry, Indiana Univ. Math. J. 25 (1976) 659.

[38] E. Calabi, On manifolds with non-negative Ricci curvature II, Notices Am. Math. Soc. 22 (1975) A205.

[39] W. Ballmann, M. Gromov and V. Schroeder, Manifolds of Nonpositive Curvature, in Progress in Mathematics 61, Springer (1985).

[40] W. Ballmann, Lectures on Spaces of Nonpositive Curvature, in DMV Seminars 25, Birkhäuser Basel (1995).

[41] A. Borel, Density and maximality of arithmetic subgroups, J. Reine Angew. Math. 244 (1966) 78.

[42] N. Seiberg and E. Witten, Electric-magnetic duality, monopole condensation, and confinement in $N=2$ supersymmetric Yang-Mills theory, Nucl. Phys. B 426 (1994) 19 [Erratum ibid. 430 (1994) 485] [hep-th/9407087] [INSPIRE]. 
[43] N. Seiberg and E. Witten, Monopoles, duality and chiral symmetry breaking in $N=2$ supersymmetric QCD, Nucl. Phys. B 431 (1994) 484 [hep-th/9408099] [InSPIRE].

[44] W. Baily and A. Borel, Compactification of arithmetic quotients of bounded symmetric domains, Ann. Math. 84 (1966) 442.

[45] A. Borel and L. Ji, Compactifications of Symmetric and Locally Symmetric Spaces, in Mathematics: Theory \& Applications, Birkäuser (2006).

[46] J.S. Milne, Shimura Varieties and Moduli, arXiv:1105.0887 and online at https://www.jmilne.org/math.

[47] J.S. Milne, Shimura Varieties and Motives, in Proceedings of Symposia in Pure Mathematics 55.2, American Mathematical Society, Providence RI U.S.A. (1994), pp. 447-523 and online at https://www.jmilne.org/math.

[48] Y. Namikawa, Toroidal compactification of Siegel spaces, in Lecture Notes in Mathematics 812, Springer, Berlin Germany (1980).

[49] C.-L. Chai, Compactification of Siegel moduli schemes, in London Mathematical Society Lecture Note Series 107, Cambridge University Press, Cambridge U.K. (1985).

[50] S.-T. Yau and Y. Zhang, The geometry on smooth toroidal compactifications of Siegel varieties, arXiv:1201.3785.

[51] S. Cecotti and C. Vafa, Theta-problem and the String Swampland, arXiv:1808.03483 [INSPIRE].

[52] C. Vafa, Evidence for F-theory, Nucl. Phys. B 469 (1996) 403 [hep-th/9602022] [InSPIRE].

[53] C. Soulé, An introduction to arithmetic groups, math.GR/0403390.

[54] I.G. Macdonald, The volume of a compact Lie group, Inven. Math. 56 (1980) 93.

[55] R.P. Langlands, The volume of the fundamental domain for some arithmetical subgroups of Chevalley groups, in Algebraic Groups and Discontinuous Subgroups, Proceedings of Symposia in Pure Mathematics 9, American Mathematical Society, Providence RI U.S.A. (1966), pp. 235-257.

[56] P. Griffiths, Topics in Transcendental Algebraic Geometry, in Annals of Mathematics Studies, Princeton University Press, Princeton NJ U.S.A. (1984).

[57] P. Deligne, Travaux de Griffiths, in Séminaire Bourbaki vol. 1969/70 Exposés 364-381, Lecture Notes in Mathematics 180, Springer (1970).

[58] J. Carlson, S. Müller-Stach and C. Peters, Period Mappings and Period Domains, second edition, in Cambridge Studies in Advanced Mathematics 168, Cambridge University Press, Cambridge U.K. (2017).

[59] P. Griffiths, Mumford-Tate groups, (2010) and online pdf version at https://publications.ias.edu/sites/default/files/Trieste.pdf.

[60] M. Green, P. Griffiths and M. Kerr, Mumford-Tate domains, Boll. Unione Mat. Ital. 3 (2010) 281 and online pdf version at https://www.math.wustl.edu/ matkerr/MTD.pdf.

[61] M. Green, P. Griffiths and M. Kerr, Mumford-Tate Groups and Domains: Their Geometry and Arithmetic, in Annals of Mathematics Studies, Princeton University Press, Princeton NJ U.S.A. (2012). 
[62] S. Cecotti and C. Vafa, Topological antitopological fusion, Nucl. Phys. B 367 (1991) 359 [INSPIRE].

[63] S. Cecotti, Geometry of $N=2$ Landau-Ginzburg families, Nucl. Phys. B 355 (1991) 755 [INSPIRE].

[64] S. Cecotti and C. Vafa, On classification of $N=2$ supersymmetric theories, Commun. Math. Phys. 158 (1993) 569 [hep-th/9211097] [INSPIRE].

[65] B. Dubrovin, Geometry and integrability of topological-antitopological fusion, Commun. Math. Phys. 152 (1993) 539 [hep-th/9206037] [INSPIRE].

[66] F. Hélein and J.C. Wood, Harmonic maps: Dedicated to the memory of James Eells, in Handbook of Global Analysis, D. Krupka and D. Saunders eds., Elsevier Science (2007), pp. 417-491.

[67] S. Helgason, Differential Geometry, Lie Groups and Symmetric Spaces, in Pure and Applied Mathematics 80, Academic Press, New York NY U.S.A. (1978).

[68] C.T. Simpson, Higgs bundles and local systems, Publ. Math. IHÉS 75 (1992) 5.

[69] K. Corlette, Archimedean superrigidity and hyperbolic geometry, Ann. Math. 135 (1992) 165.

[70] J.A. Carlson and D. Toledo, Harmonic mappings of Kähler manifolds to locally symmetric spaces, Publ. Math. IHÉS 69 (1989) 173.

[71] J.H. Sampson, Applications of harmonic maps to Kähler geometry, in Contemporary Mathematics 49, American Mathematical Society, Providence RI U.S.A. (1986), pp. 125-134.

[72] W. Lerche, C. Vafa and N.P. Warner, Chiral Rings in $N=2$ Superconformal Theories, Nucl. Phys. B 324 (1989) 427 [INSPIRE].

[73] A. Skowroński, Periodicity in representation theory of algebras, (2006) and online pdf version at https://webusers.imj-prg.fr/ bernhard.keller/ictp2006/lecturenotes/skowronski.pdf.

[74] K. Hori, A. Iqbal and C. Vafa, D-branes and mirror symmetry, hep-th/0005247 [INSPIRE].

[75] P. Griffiths and W. Schmid, Locally homogeneous complex manifolds, Acta Math. 123 (1969) 253.

[76] A. Sen, Black hole entropy function and the attractor mechanism in higher derivative gravity, JHEP 09 (2005) 038 [hep-th/0506177] [INSPIRE].

[77] A. Sen, Entropy function for heterotic black holes, JHEP 03 (2006) 008 [hep-th/0508042] [INSPIRE].

[78] A. Sen, Black Hole Entropy Function, Attractors and Precision Counting of Microstates, Gen. Rel. Grav. 40 (2008) 2249 [arXiv:0708.1270] [INSPIRE].

[79] W. Schmid, Variation of Hodge Structure: the singularities of the period mapping, Inv. Math. 22 (1973) 211.

[80] S. Ferrara, R. Kallosh and A. Strominger, $N=2$ extremal black holes, Phys. Rev. D 52 (1995) R5412(R) [hep-th/9508072] [INSPIRE].

[81] A. Strominger, Macroscopic entropy of $N=2$ extremal black holes, Phys. Lett. B 383 (1996) 39 [hep-th/9602111] [INSPIRE].

[82] S. Ferrara and R. Kallosh, Supersymmetry and attractors, Phys. Rev. D 54 (1996) 1514 [hep-th/9602136] [INSPIRE]. 
[83] G.W. Moore, Arithmetic and attractors, hep-th/9807087 [INSPIRE].

[84] H. Ooguri, A. Strominger and C. Vafa, Black hole attractors and the topological string, Phys. Rev. D 70 (2004) 106007 [hep-th/0405146] [INSPIRE].

[85] K. Goldstein, N. Iizuka, R.P. Jena and S.P. Trivedi, Non-supersymmetric attractors, Phys. Rev. D 72 (2005) 124021 [hep-th/0507096] [INSPIRE].

[86] J. de Boer, J. Manschot, K. Papadodimas and E. Verlinde, The Chiral ring of $A d S_{3} / C F T_{2}$ and the attractor mechanism, JHEP 03 (2009) 030 [arXiv:0809.0507] [INSPIRE].

[87] J. Eells and J. Sampson, Harmonic mappings of Riemannian manifolds, Am. J. Math. 86 (1964) 109.

[88] K. Corlette, Flat G-bundles with canonical metrics, J. Diff. Geom. 28 (1988) 361.

[89] D.H. Gottlieb, Covering transformations and universal fibrations, Illinois J. Math. 13 (1969) 432.

[90] V.L. Hansen, On a theorem of Al'ber on spaces of maps, J. Diff. Geom. 12 (1977) 565.

[91] J. Jöst, Riemannian Geometry and Geometric Analysis, seventh edition, in Universitext, Springer International Publishing (2017).

[92] S.I. Al'ber, Spaces of mappings into a manifold with negative curvature, Dokl. Akad. Nauk SSSR 178 (1968) 13 [Soviet Math. Dokl. 9 (1968) 6].

[93] S.I. Al'ber, The topology of functional manifolds and the calculus of variations in the large, Russ. Math. Surv. 25 (1970) 51.

[94] D. Gaiotto, G.W. Moore and A. Neitzke, Four-dimensional wall-crossing via three-dimensional field theory, Commun. Math. Phys. 299 (2010) 163 [arXiv:0807.4723] [INSPIRE].

[95] C. Closset, T.T. Dumitrescu, G. Festuccia and Z. Komargodski, The Geometry of Supersymmetric Partition Functions, JHEP 01 (2014) 124 [arXiv:1309.5876] [INSPIRE].

[96] J.S. Milne, Algebraic Groups. The theory of group schemes of finite type over a field, Cambridge University Press, Cambridge U.K. (2017) [https://doi.org/10.1017/9781316711736].

[97] A. Hatcher, Algebraic topology, Cambridge University Press, Cambridge U.K. (2002).

[98] E. Palti, C. Vafa and T. Weigand, Supersymmetric Protection and the Swampland, JHEP 06 (2020) 168 [arXiv: 2003.10452] [inSPIRE]. 